ADDITIVE MANUFACTURING OF HIGH PERFORMANCE FLEXIBLE THERMOELECTRIC GENERATORS USING NANOPARTICLE INKS

by

Tony Valayil Varghese

\author{
A dissertation \\ submitted in partial fulfillment \\ of the requirements for the degree of \\ Doctor of Philosophy in Materials Science and Engineering \\ Boise State University
}

May 2019 
(C) 2019

Tony Valayil Varghese

ALL RIGHTS RESERVED 


\section{BOISE STATE UNIVERSITY GRADUATE COLLEGE \\ DEFENSE COMMITTEE AND FINAL READING APPROVALS}

of the dissertation submitted by

Tony Valayil Varghese

Dissertation Title: Additive Manufacturing of High Performance Flexible Thermoelectric Generators Using Nanoparticle Inks

Date of Final Oral Examination: $\quad 25$ April 2019

The following individuals read and discussed the dissertation submitted by student Tony Valayil Varghese, and they evaluated the presentation and response to questions during the final oral examination. They found that the student passed the final oral examination.

Yanliang Zhang, Ph.D.

David Estrada, Ph.D.

Claire Xiong, Ph.D.

Rutvik Jatin Mehta, Ph.D.
Chair, Supervisory Committee

Co-Chair, Supervisory Committee

Member, Supervisory Committee

Member, Supervisory Committee

The final reading approval of the dissertation was granted by Yanliang Zhang, Ph.D., Chair of the Supervisory Committee. The dissertation was approved for the Graduate College. 
This dissertation is dedicated to my family and friends for their continuous support and motivation over the years. 


\section{ACKNOWLEDGEMENTS}

First and foremost, I would like to express my sincere gratitude to my academic advisors, Dr. Yanliang Zhang and Dr. David Estrada for providing me with this opportunity. Their support and guidance had an impactful influence on both my academic career and personal life. Additionally, I would like to extend my thanks to my committee members Dr. Claire Xiong and Dr. Rutvik J Mehta for the successful completion of my dissertation and their mentorship.

I am grateful to Dr. Alex Punnoose and Dr. Don Plumlee for providing access to their lab facilities and instruments. I am also grateful to Dr. Madhu Kongara for all the instrument training and mentoring. I greatly appreciated the help and support I received from Peter Miranda and Travis Gabel, throughout my work. Their willingness to help whenever there is a problem made my work easier and better.

I want to thank all my previous lab members and my friends; Ryan Stevenson, Courtney Hollar, Chao Han, Luke Schoensee, Nicolas Kempf, Joseph Richardson and Pasindu Gamarachi for helping me with my work in and out of the lab. Their encouragement and help both have been instrumental in the success of my work and progression of my dissertation. I want extend my gratitude for support and help I got from Chaochao Dun and Mortaza Saeidi-Javash and to our collaborators Dr. Rahul Panat and Roozbeh Danaei. I am thankful for the opportunity to work with these great people and their support for my works. 
I want to thank Dr. Dave Estrada and Dr. Harish Subbaraman for adopting me to ANML group and introducing me to a great group of people. I appreciate the emotional support and their willingness to help any time in need. I really appreciate the motivation I got from my friends at ANML group and I want to extend my thanks to Twinkle Pandi, Kiyo Fujimoto and Binay Joshi for their help in the progress of my work.

My sincere thanks also go to all the material science staff for their support and help, especially Chad Watson, Jessica Economy, Stephanie Moran and Jamie Hayward also Justin Larson in the Mechanical and Biomedical Engineering Department for being an integral part in the progress of this work.

I want to extend my thanks to Raju Chacko, Elizabeth Chacko, Dr. Stephen Thomas, Anuya Thomas, George Mazhuvancheri and Sheetal George my Boise family for all the wonderful memories and giving a home away from home experience. Also I want to thank Frack Sanchez and Tonya Vorkel for their personal support and motivation though out past years.

Last but not least I thank my father V.M Varghese, my mother Mercy Varghese for their unconditional love and encouragement without it, I wouldn't have followed my dreams. I want to thank Tojo Varghese, Flemy Varghese, Lidiya Varghese and Bijin Babu for their support, love and encouragement for all my decisions to pursue my dreams. Finally, I want to thank Meriya Sanchez for your support and love, I owe much of my success to your love and encouragement. You have given me the motivation and determination to pursue my dreams through all the difficult times. 


\begin{abstract}
Flexible thermoelectric devices are attractive power sources for the growing demand of flexible electronics and sensors. Thermoelectric generators have an advantage due to no moving parts, silent operation and constant power production with a thermal gradient.

Conventional thermoelectric devices are rigid and fabricated using complex and relatively costly manufacturing processes, presenting a barrier to increase the market share of this technology. To overcome such barriers, this work focuses on developing near ambient-temperature flexible thermoelectric generators using relatively low-cost additive manufacturing processes. A screen printable ink was developed for transforming nanoparticle ink into high-performance flexible thermoelectric generators with a peak thermoelectric figure of merit of 0.43 and 1 for the n-type and p-type materials respectively. Additionally, thermoelectric material properties were further improved using a liquid phase sintering method, which resulted in bulk like performance for printed flexible devices. Lastly, aerosol jet printable inks and photonic sintering processes were developed to highlight the potential for large scale roll-to-roll manufacturing and 3D conformal printing of thermoelectric generators.
\end{abstract}




\section{TABLE OF CONTENTS}

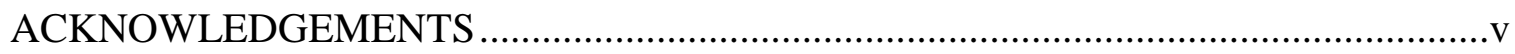

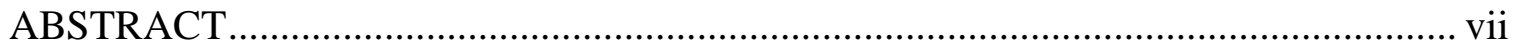

TABLE OF CONTENTS ......................................................................................... vii

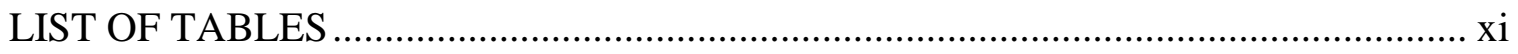

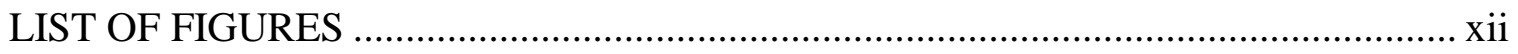

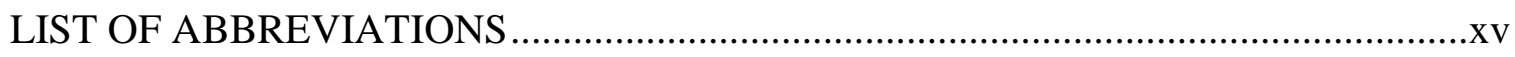

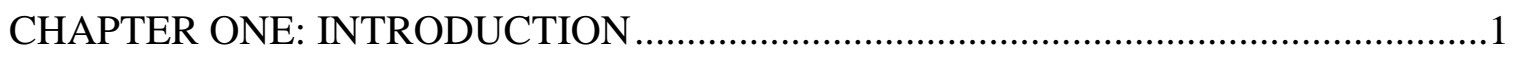

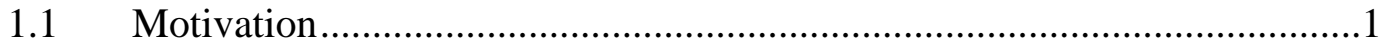

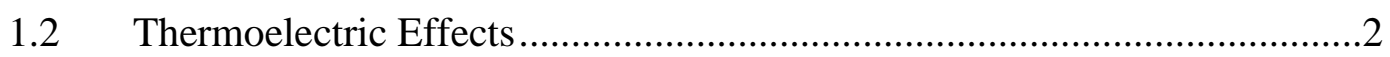

$1.3 \quad$ Thermoelectric Materials ....................................................................4

1.3.1 Nano structuring of Thermoelectric Materials ..................................5

$1.4 \quad$ Thermoelectric Manufacturing and Limitations ........................................

1.4.1 Bulk Device Manufacturing .......................................................

1.4.2 Thin Film Devices .................................................................

1.5 Additive Printing and Sintering ........................................................

$1.6 \quad$ Flexible Thermoelectric Generators (fTEG) ………................................

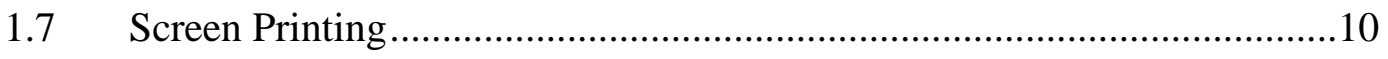

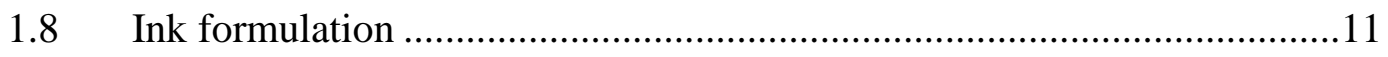

$1.9 \quad$ Aerosol Jet Printing..............................................................................14 


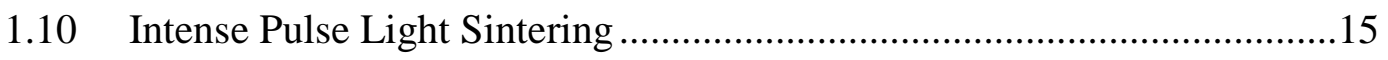

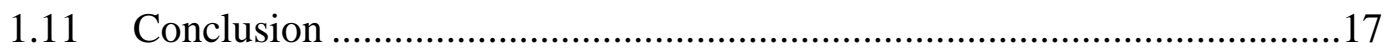

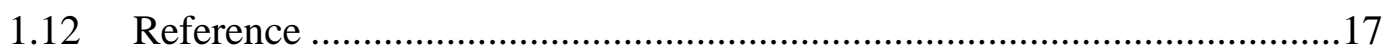

CHAPTER TWO: HIGH-PERFORMANCE AND FLEXIBLE THERMOELECTRIC FILMS BY SCREEN PRINTING SOLUTION-PROCESSED NANOPLATE

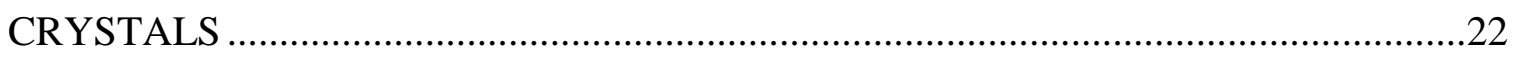

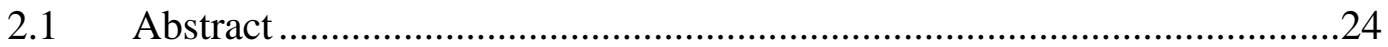

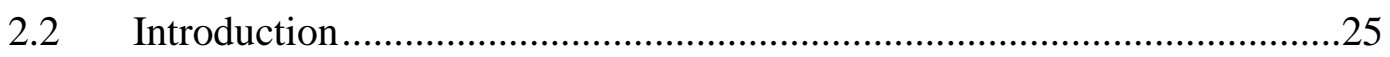

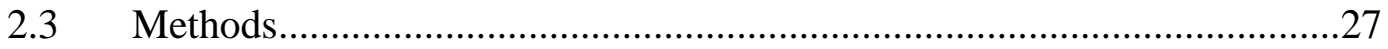

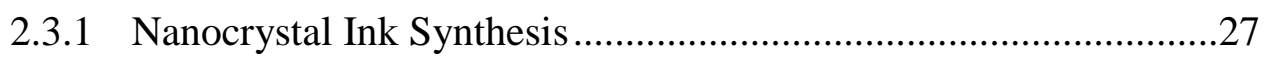

2.3.2 Flexible Film Fabrication ..............................................................28

2.3.3 Thermoelectric Property Measurement ...........................................28

$2.4 \quad$ Results and Discussion .....................................................................

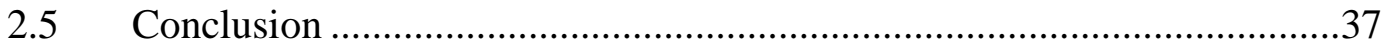

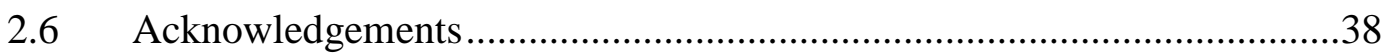

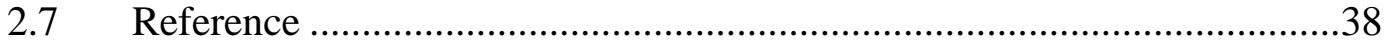

CHAPTER THREE: BULK LIKE THERMOELECTRIC PERFORMANCES OF FLEXIBLE DEVICES BY SCALABLE PRINTING AND LIQUID-PHASE SINTERING

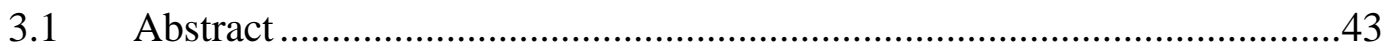

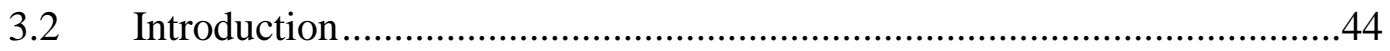

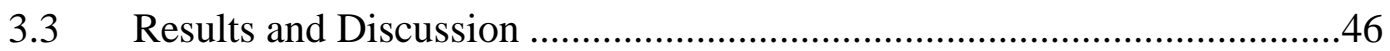

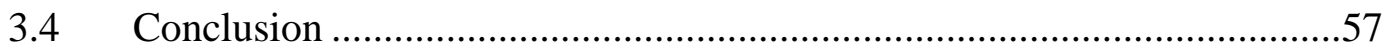

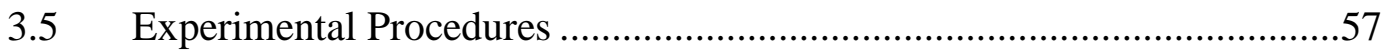

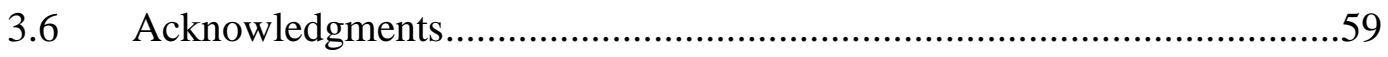


$3.7 \quad$ References:

CHAPTER FOUR - AEROSOL JET PRINTING AND PHOTONIC SINTERING OF COLLOIDAL THERMOELECTRIC NANOCRYSTALS..............................................66

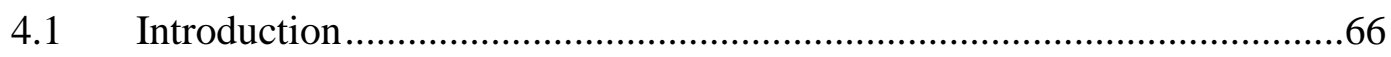

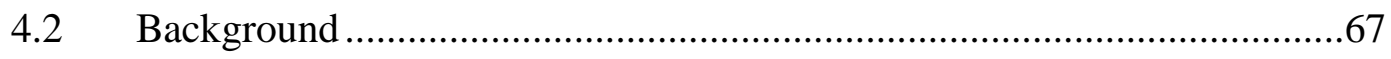

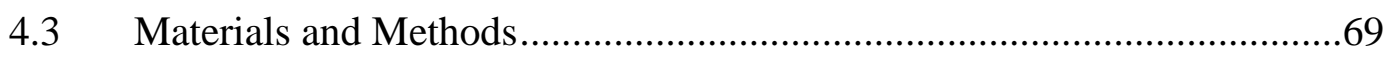

4.3.1 Thermoelectric Ink for Aerosol Jet Printing ..................................69

4.3.2 Fabrication of Aerosol Jet Printed Thermoelectric Films ...............69

4.3.3 Photonic Sintering of The Printed Films.........................................70

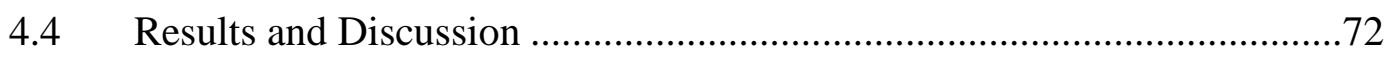

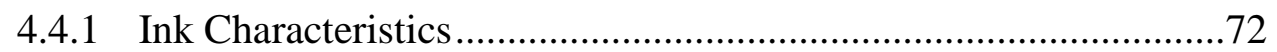

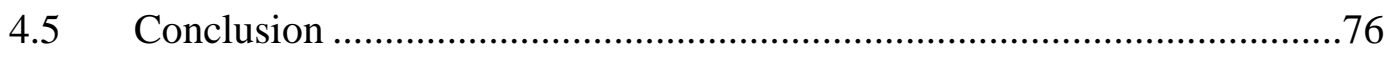

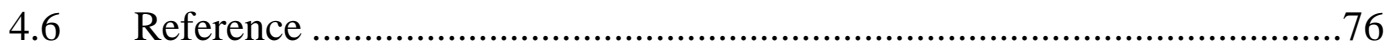

CHAPTER 5 SUMMARY AND FUTURE WORK ……………................................78

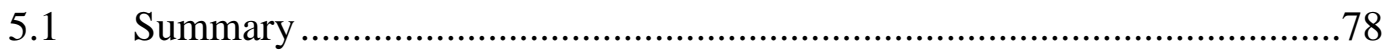

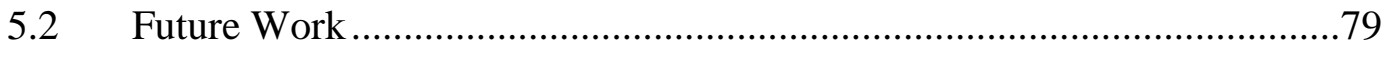

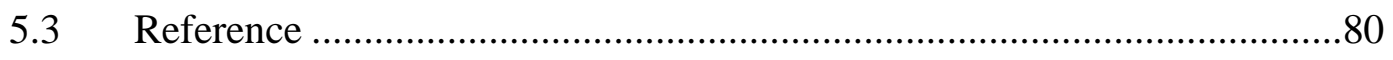




\section{LIST OF TABLES}

Table 2. 1 Hall measurement result of the BiSeTe film and pellet .......................... 31

Table 2. 2 The thermoelectric performance comparison between our work and previous reported n-type flexible thermoelectric films......................... 36

Table 3. 1 Comparison of room-temperature thermoelectric performance between this work and previous best reported values of p-type flexible thermoelectric films. .................................................................... 55

Table 4. 1 Summary of the aerosol jet printing parameters ..................................... 71 


\section{LIST OF FIGURES}

Figure 1. 1 A model representing thermoelectric energy generation using Seebeck effect

Figure 1.2 Optimizing the thermoelectric properties as a function of carrier concentration. Image courtesy () Thermoelectrics group Northwestern Materials Science and Engineering.............................................................. 5

Figure 1.3 Electronic density of states (a) Bulk 3D crystalline semiconductor (b) 2D

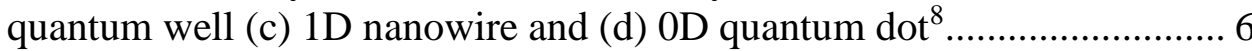

Figure 1.4 Peak ZT comparison of some of the best known thermoelectric material as a function of temperature ${ }^{12}$ (a) P-type thermoelectric materials and (b) Ntype thermoelectric material .................................................................. 7

Figure 1.5 Visual example of how screen printers distribute inks onto substrates ${ }^{27} . .11$

Figure 1.6 Schematic representation of Aerosol Jet Printing $\odot$ Optomec .................. 14

Figure 1.7 (a-e) Particle coalescence calculated by COMSOL modeling and comparing to the corresponding SEM images of the samples. ${ }^{39}$ 16

Figure 2. 1 Schematic illustration of overall fabrication process for the flexible ....... 26

Figure 2. 2 Schematic of Hall measurement sample by Four Wire method. .............. 30

Figure 2. 3 SEM images of (a) the $\mathrm{Bi}_{2} \mathrm{Te}_{2.8} \mathrm{Se}_{0.2}$ nanocrystals and (b) the cross section of the flexible film. 31

Figure 2. 4 Thickness measurement for film using SEM.......................................... 32

Figure 2.5 Temperature-dependent (a) Electrical conductivity (b) Seebeck coefficient (c) Thermal conductivity and (d) ZT of a flexible film and a reference pellet as a control pellet. 33

Figure 2.6 (a) sample holder for measuring in-plane thermal conductivity, (b) A thermoelectric film sample mounted in the sample holder. 34 
Figure 2. 7 Percentage increases of electrical resistivity of the flexible film vs. number of bending cycles. The inset is a flexible film bended on a glass tube of 14 mm diameter. 37

Figure 3. 1 Converting $\mathrm{Bi}_{0.4} \mathrm{Sb}_{1.6} \mathrm{Te}_{3}(\mathrm{BST})$ nanoparticle ink into a flexible thermoelectric generator using a scalable and low-cost screen-printing and liquid-phase sintering process...................................................... 46

Figure 3. 2 X-ray diffraction pattern comparison between BST and BST:T samples, which verifies the presence of excess elemental tellurium (marked as blue stars) in the BST:T samples that is in agreement with the EDS.

Figure 3. 3 SEM images of (a) printed $\mathrm{Bi}_{0.4} \mathrm{Sb}_{1.6} \mathrm{Te}_{3}$ films with 8 wt.\% Te and (b) printed pure $\mathrm{Bi}_{0.4} \mathrm{Sb}_{1.6} \mathrm{Te}_{3}$ films. The corresponding energy-dispersive $\mathrm{X}$ ray spectroscopy (EDS) mapping is given in (c) and (d), respectively .... 48

Figure 3. 4 (a) SEM of BST:T film with (b) the corresponding EDS analysis, (c) detailed elements ratios for the part I and II as marked in (a). It is observed that the re-precipitation of Te occurs, which increase the film density that is helpful for the increased electrical conductivity. The main body (part II) has the same element ratios of $\mathrm{Bi}-\mathrm{Sb}-\mathrm{Te}$ as the original particles $\left(\mathrm{Bi}_{0.4} \mathrm{Sb}_{1.6} \mathrm{Te}_{3}\right)$.

Figure 3.5 SEM images of the BST films without (a) and with (b) additional Te with lower magnifications. All scar bars are $25 \mu \mathrm{m}$. It is shown that the BST:T films are more dense with less porosity compared with that of BST films

Figure 3. 6 Temperature-dependent (a) electrical conductivity, (b) Seebeck coefficient, and (c) power factor of both BST and BST:T films. (d) Normalized resistance of flexible films vs. number of bending cycles with a $10 \mathrm{~mm}$ bending radius for both BST and BST:T films. Here, $\mathrm{R}_{0}$ and $\mathrm{R}$ are the resistances before and after bending.

Figure $3.7 \quad$ (a) Schematic of the Angstrom measurement setup; (b) measured temperature waves of the two thermocouples on the sample 53

Figure 3. 8 Performance of a flexible TE device fabricated by the screen-printing and liquid-phase sintering process: (a) calculated and experimental open circuit voltage vs. temperature differences $(\Delta \mathrm{T})$ (Inset: photo of the printed device); (b) device operating voltage vs. current tested at various $\Delta \mathrm{T}$; (c) calculated and experimental electrical power density tested at various $\Delta \mathrm{T}$; and (d) power output vs. electrical current at various $\Delta \mathrm{T}$. 56

Figure 4. 1 (a) Perimeter filled AJP prints (b) Serpentine filled AJP prints with 30$40 \%$ overlapping 
Figure 4. 2 Contact angle measurements of the AJP thermoelectric inks (a) cleaned Kapton substrate (b) Oxygen plasma treated Kapton substrate ................ 73

Figure 4. 3 (a) \& (b) AJP printed samples on Kapton and Photopaper,(c) AJP printed photonic sintered N-type thermoelectric device,(d) 3D conformal printed thermoelectric devices on silver contacts.

Figure 4. 4 3D mapping of the aerosol jet printed sample (a) dried sample with no pressure applied (b) sample after 10Klbf force applied using a hydraulic press.

Figure 4. 5 SEM images (a) surface images of $\mathrm{Bi}_{2} \mathrm{Te}_{3}$ photonic sintered sample and (b-c) cross section of a printed film on polyimide substrate AJP printed and photonic sintered.

Figure 4.6 (a) electrical conductivity and seebeck coefficient as a function of number of pulses (b) power factor vs number of pulses. 76 


\section{LIST OF ABBREVIATIONS}

AI

$5 \mathrm{G}$

IoT

$3 \mathrm{D}$

2D

TEG

ZT

$\Delta \mathrm{T}$

$\Delta \mathrm{V}$

$\alpha$

TEC

$\sigma$

$\kappa$

$\mathrm{L}$

n

$\mu$

PF

OD

1D

fTEG
Artificial Intelligence

$5^{\text {th }}$ Generation cellular mobile communication

Internet of things

Three-dimensional

Two- dimensional

Thermoelectric Generator

Figure of Merit

Temperature Change

Potential Difference

Seebeck Coefficient

Thermoelectric Cooler

Electrical conductivity

Thermal conductivity

Lorenz number

Number of charge carriers

Mobility of charge carriers

Power Factor

Zero-dimensional

One-dimensional

Flexible Thermoelectric Generator 


\begin{tabular}{|c|c|}
\hline $\mathrm{cP}$ & Centipoise \\
\hline$\mu \mathrm{m}$ & Micrometer \\
\hline BST & Bismuth Antimony Telluride \\
\hline BST:T & Bismuth Antimony Telluride with $8 \%$ tellurium \\
\hline${ }^{\circ} \mathrm{C}$ & Degree Celsius \\
\hline PEDOT:PSS & Poly(3,4-ethylenedioxythiophene): Polystyrene sulfonate \\
\hline PAA & Poly (acrylic acid) \\
\hline AJP & Aerosol Jet Printing \\
\hline MWCNT & Multi wall carbon nanotubes \\
\hline IPL & Intense Pulse Light \\
\hline UV & Ultraviolet \\
\hline SEM & Scanning Electron Microscope \\
\hline TEM & Transmission Electron Microscope \\
\hline TGA & Thioglycolic acid \\
\hline $\mathrm{P}$ & Power \\
\hline I & Current \\
\hline $\mathrm{R}$ & Resistance \\
\hline XRD & $\mathrm{X}$-ray diffraction \\
\hline $\mathrm{P}$ & Density \\
\hline EDS & Energy Dispersive Spectroscopy \\
\hline
\end{tabular}




\section{CHAPTER ONE: INTRODUCTION}

\subsection{Motivation}

Advancement in artificial intelligence (AI) and the coming of $5^{\text {th }}$ generation $(5 \mathrm{G})$ cellular mobile communication devices promotes the rise of advanced wireless communication and wearable devices for Internet of Things (IoT). A systematic and efficient progress of this technology is only possible with deploying a large number of sensors and devices that can communicate continuously; but a continuous power consumption is a bottleneck for the life and comfort of these devices. Current technology depends largely on coin cell batteries because of their compact nature in wireless communication devices but which have to be replaced every six months or a year depending on usage. Whereas using rechargeable lithium ion batteries requires, larger batteries to reduce frequent charging and difficulties in powering make it costly and inefficient especially for a large number of devices.

Recently renewable energy powered battery sources are becoming a rising star in overcoming these limitations. Main renewable power sources available for rechargeable batteries are solar, piezoelectric and thermoelectric energy harvesters ${ }^{1-4}$. Among these, thermoelectric generators have an advantage over other two modes of charging for low power electronic devices. Thermoelectric generators can function with natural states by

utilizing the temperature difference between surfaces and ambient temperature ${ }^{5-7}$ day and night without any moving parts involved. 
Thermoelectric generators (TEGs) produce electrical power using thermal energy as long as a temperature gradient exists. In the last two decades, tremendous improvement in thermoelectric material figure of merit (ZT) has been obtained by Nano structuring of the materials ${ }^{8-10}$. The limited durability and high cost for transforming the nanostructured materials into functional devices are still a barrier in bringing this technology into the commercial market ${ }^{11}$. This work focuses on transforming thermoelectric nanocrystals to highly functional thermoelectric devices using an additive printing and sintering process.

\subsection{Thermoelectric Effects}

Thermoelectric effect describes direct conversion of thermal energy gradient into electrical energy or vice versa. A thermoelectric generator produces electrical voltage when a temperature gradient exists between its two junctions. Thermoelectric materials are useful in applications such as energy harvesting, cooling and temperature sensing. The fundamental principles behind every thermoelectric application are Seebeck, Peltier and Thomson effects.

When two materials are connected electrically in a series and a temperature difference is applied between the two junctions, electrons at the hot junction will diffuse into the cold junction creating an electrical potential by virtue of a materials property called the Seebeck effect, as represented in Figure 1.1. The Seebeck coefficient value is different for different materials, generally most of the thermoelectric materials are highly doped semiconductors and semi-metals which has a Seebeck coefficient higher than metals and lower than insulators. 


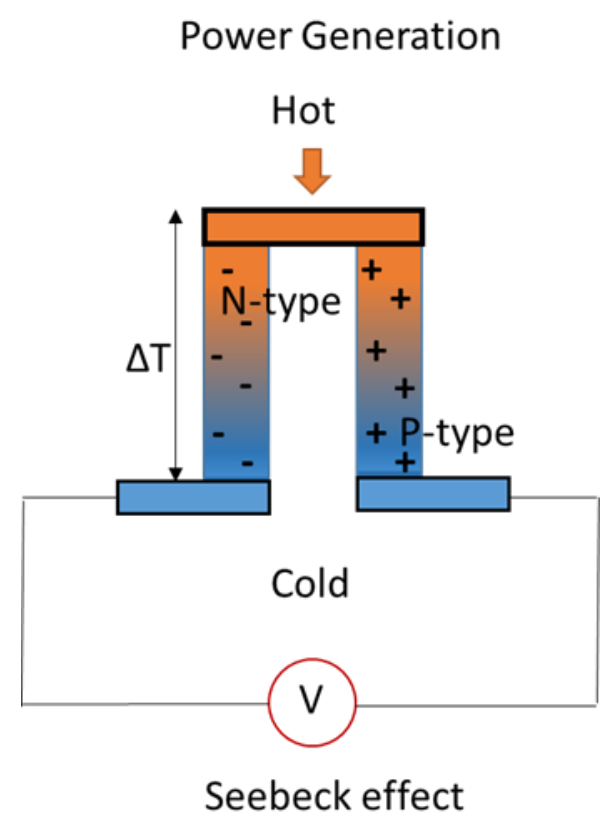

Figure 1.1 A model representing thermoelectric energy generation using Seebeck effect

A material produces a Seebeck voltage due to a change in carrier concentration, charge carriers are generated at the hot side and diffuse to the cold side creating an internal electric field. This potential difference at that temperature corresponds to the Seebeck voltage produced and it varies with the temperature differences. The Seebeck coefficient $(\alpha)$ can be expressed in terms of electric potential difference $(\Delta \mathrm{V})$ and temperature gradient $(\Delta \mathrm{T})$ across the material junctions

$$
\alpha=-\frac{\Delta V}{\Delta T}
$$

In case of a thermoelectric coolers (TEC), an electric current is passed between two junctions and a temperature gradient is observed between the junctions by virtue of the Peltier effect. The electric current that flows will result in heat absorption (cold) at one junction and heat dissipation at the other end (hot) resulting in a temperature gradient. However, when a single material undergoes a temperature difference and an 
electric current passes through it simultaneously a heat power is absorbed or evolved along the material known as the Thomson effect.

\subsection{Thermoelectric Materials}

Conversion of thermal energy to electrical energy using thermoelectric material involves no moving parts, which make very useful systems in a lot of applications. The performance of thermoelectric material is often expressed using a dimensionless property figure of merit (ZT) as given below

$$
Z T=\frac{\sigma \alpha^{2} T}{\kappa_{\text {total }}}=\frac{\sigma \alpha^{2} T}{\kappa_{\text {lattice }}+\kappa_{e}}
$$

The ZT of a thermoelectric material is directly proportional to electrical conductivity of the material $(\sigma)$, Seebeck coefficient $(\alpha)$ and inversely proportional to its thermal conductivity $\left(\kappa_{\text {total }}\right)$, which is the sum of lattice phonons and electrons/holes $\left(\kappa_{\text {lattice }}+\kappa_{\text {electronic }}\right)$ at that temperature $(\mathrm{T})$. To maximize the $\mathrm{ZT}$, it is essential to have a high electrical conductivity and lower thermal conductivity. But the electronic thermal conductivity $\left(\kappa_{\mathrm{e}}\right)$ is directly related to the electrical conductivity $(\sigma)$ through WiedemannFranz law. $\kappa_{e}=L \sigma T=n e \mu L T$ Where $\mathrm{L}$ is the Lorenz factor, $\mathrm{n}$ is the number of charge carriers and $\mu$ is the mobility of the material. The term on the nominator $\sigma \alpha^{2}$ is defined as the power factor (PF), which correlates to the electrical energy generation of the thermoelectric material. All three of these properties are interrelated, in order to obtain a high ZT value we have to maximize the $\boldsymbol{\sigma}, \alpha$ and minimize the $\boldsymbol{\kappa}$ which is very difficult to obtain. Figure 1.2 represents optimum thermoelectric properties to attain a high power factor as a function of carrier concentrations. 


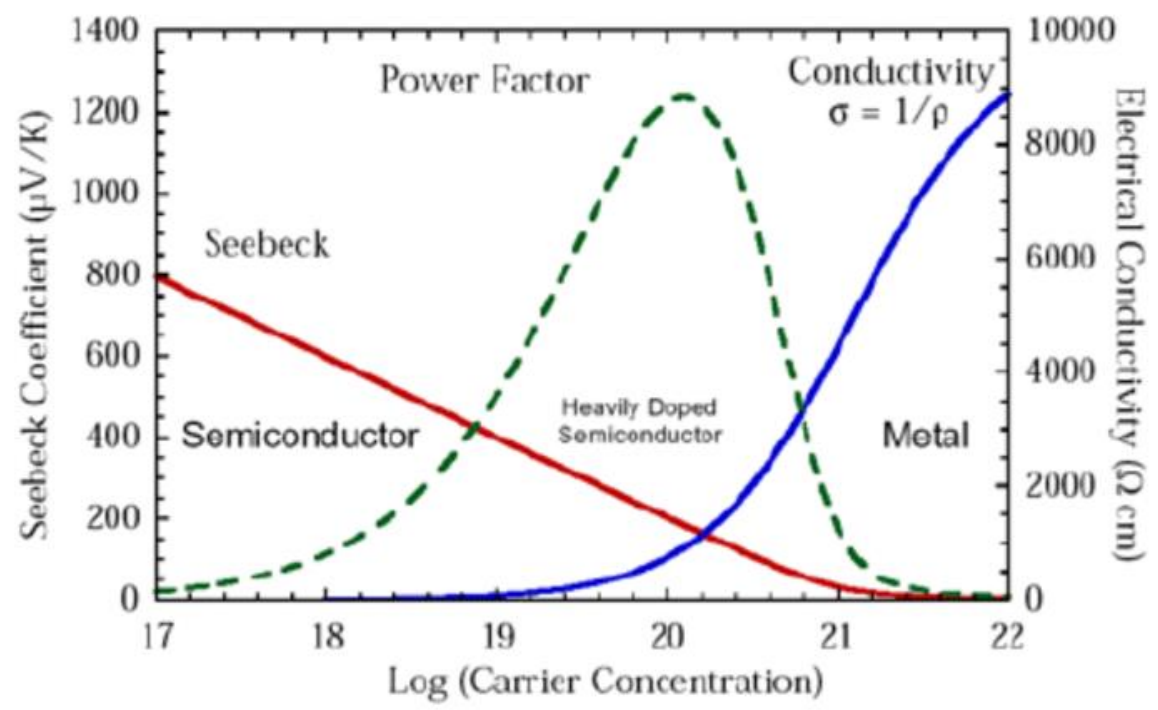

Figure 1.2 Optimizing the thermoelectric properties as a function of carrier concentration. Image courtesy (C) Thermoelectrics group Northwestern Materials Science and Engineering

\subsubsection{Nano structuring of Thermoelectric Materials}

The performance enhancement of thermoelectric material was limited due to the interdependence of the each thermoelectric property until in 1993 Dresselhaus proposed a dimensional control or Nano structuring of the thermoelectric materials. ${ }^{8}$ They proposed it is possible to individually tune the thermal and electrical properties of thermoelectric materials by nanostructuring and thereby varying the electronic density states and mean free paths for electrical and thermal energy carriers. When the material size is reduced from 3D bulk size to 2D (quantum wells) to $1 \mathrm{D}$ (quantum wires) and finally to $0 \mathrm{D}$ (quantum dots) the shape of density states changes as shown in Figure 1.3, altering the thermoelectric properties of the material. 


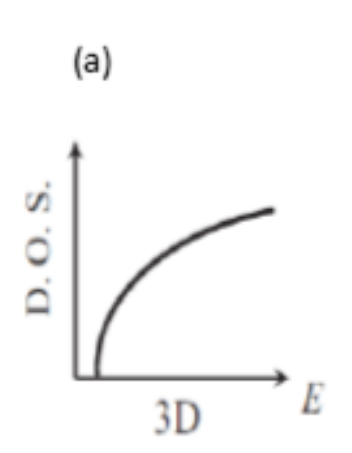

Bulk Semiconductor (b)

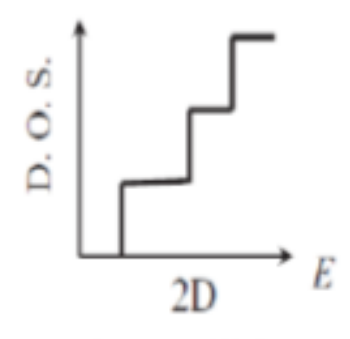

Quantum Well (c)

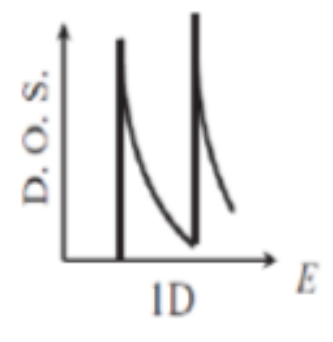

Quantum Wire (d)

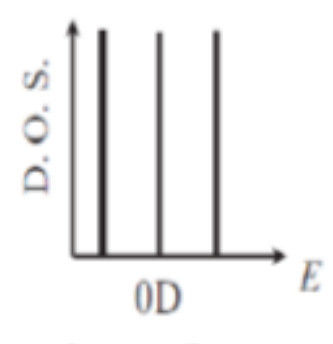

Quantum Dot

Figure 1.3 Electronic density of states (a) Bulk 3D crystalline semiconductor (b) 2D quantum well (c) 1D nanowire and (d) 0D quantum $\operatorname{dot}^{8}$

Nano structuring reduces the lattice thermal conductivity of material, reducing the phonon mean free path and scattering the larger phonon waves. The interface between the nanostructures scatters phonons more strongly than scattering electrons, allowing a separate control of lattice and electronic conductivity. Even though the interfaces can scatter longer electrons and phonon waves, grain boundaries have a smaller effect on electronic conductivity because the majority of the electrons have small mean free path compared to high mean free path acoustic phonons there by helping to improve ZT of the materials. The ZT value of thermoelectric material varies as a function of its temperature as represented in Figure 1.4. A suitable material has to be selected to work in a particular temperature based on their peak ZT values. Based on the peak ZT value temperature thermoelectric materials are broadly classified in to three categories: low temperature $\left(15^{\circ} \mathrm{C}\right.$ to $\left.200^{\circ} \mathrm{C}\right)$, medium temperature $\left(200^{\circ} \mathrm{C}\right.$ to $\left.800^{\circ} \mathrm{C}\right)$ and high temperature $\left(>800^{\circ} \mathrm{C}\right)$ thermoelectric material. This work focuses on harvesting small temperature differences using flexible thermoelectric energy harvesters (fTEGs) manufactured by additive printing and sintering. 

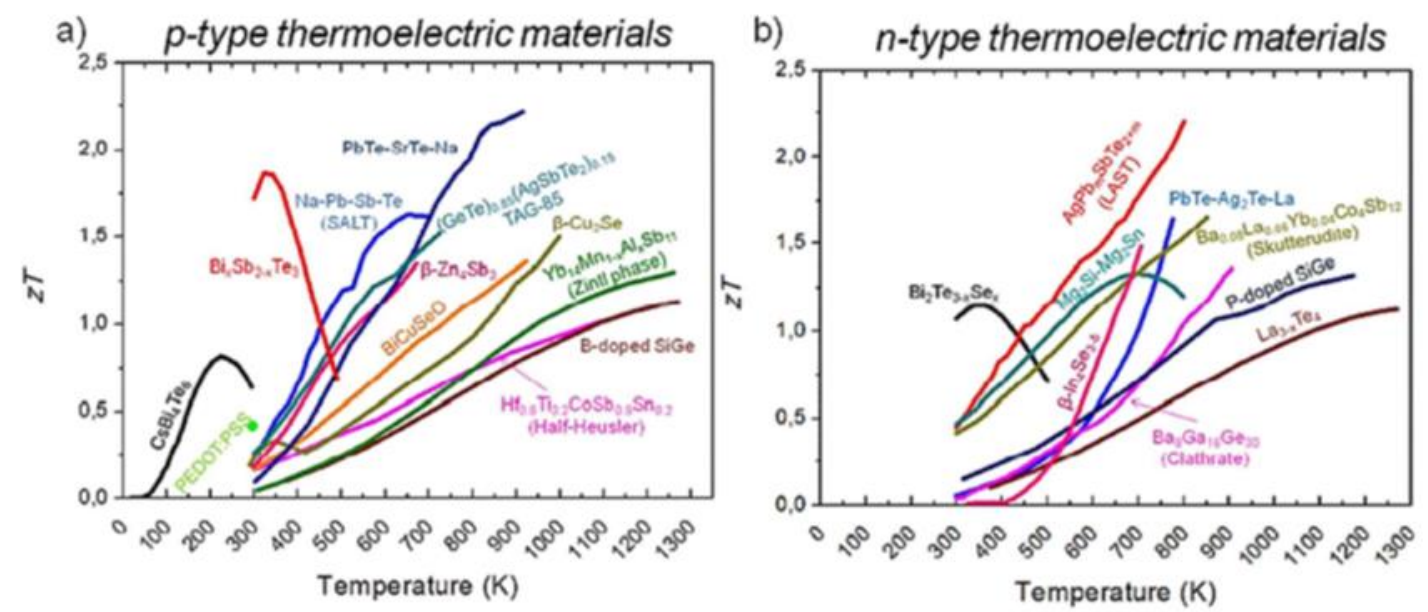

Figure 1. 4 Peak ZT comparison of some of the best known thermoelectric material as a function of temperature ${ }^{12}$ (a) P-type thermoelectric materials and (b) N-type thermoelectric material

\subsection{Thermoelectric Manufacturing and Limitations}

Thermoelectric materials manufacturing procedure varies according to the application of TEG. There are main two classes of thermoelectric generator bulk devices and thin film devices with varying manufacturing methods and limitations to fabricate.

\subsubsection{Bulk Device Manufacturing}

Bulk thermoelectric generators are widely used in thermoelectric application because of their high efficiency in converting the heat energy. The manufacturing of bulk thermoelectric generators starts with the manufacturing of thermoelectric legs (elements) $\mathrm{N} \& \mathrm{P}$ as shown in Figure 1.1. The thermoelectric discs are cut in to bars and then diced in to legs according to the dimensional requirements ${ }^{13}$. These legs are bonded electrically in series and thermally in parallel to metal contacts using pick and place technique.

Since thermoelectric materials are very brittle cutting of small elements and high aspect ratio for TEG legs are highly challenging. The cross- sectional area of the elements are also limited by the manufacturing process and efficiency requirements. The 
manufacturing process for these devices include the pick and place method, which is a labor intensive and costly process. The cost of a TEG is mainly associated with the manufacturing process of the devices, because of the lack of automation and limitation in making low density arrays with low aspect ratio ${ }^{13}$

\subsubsection{Thin Film Devices}

Thin films are manufactured to overcome the size limitations of bulk manufacturing. Thin films and micro pellets were manufactured using semiconductor micro fabrication techniques such as sputtering and thermal evaporation techniques. ${ }^{14} \mathrm{~A}$ limitation of this thin film fabrication is that thermoelectric elements taller than a few microns is very expensive and time consuming to fabricate. Conventional thin film fabrication is complicated and expensive and limited to microscale.

\subsection{Additive Printing and Sintering}

The bottle neck in the fabrication of a cost effective and efficient thermoelectric generator is the manufacturing limitations of thermoelectric generators. Additive manufacturing techniques offer an opportunity to improve the manufacturing process in a cost effective manner maintaining the efficiency of the materials. Direct printing of thermoelectric material on to substrates are an easy way to transform materials to device manufacturing. Different printing techniques have been used in the fabrication of thermoelectric generators depending on dimensional requirements; direct writing, screen printing, spray printing and Aerosol jet printing are a few of them. Printing involves deposition of inks with active thermoelectric materials and suitable additives and solvents. It eliminates the expensive process steps such as lithography, cutting and dicing and sputtering and etching. It utilizes additive processing steps which eliminates the 
material wastage and cost of manufacturing and also saves time. It is easy to fabricate a high aspect ratio and high density arrays using printing in a highly scalable and cost effective way.

Different printers are selected based on particle loading, Ink viscosity, sample dimensions and 2D or 3D printing requirements. Direct writing utilizes ink viscosities of $100-10000 \mathrm{cP}$ and is capable of both $2 \mathrm{D}$ and 3D printing but one disadvantage of this process is the low through-put for the device fabrications. Whereas screen printing is capable of large scale 2D manufacturing of devices utilizing ink viscosities up to 20000 $\mathrm{cP}$ and is limited by sample dimensions and planar printing. Finally, aerosol jet printing is capable of printing very fine features in both 2D and 3D objects with precision and utilizes ink viscosities in the range of $1-1000 \mathrm{cp}$ but they are slow processes and creating larger samples is difficult. A suitable printing process is selected according to the application of the devices and cost of fabrication.

\subsection{Flexible Thermoelectric Generators (fTEG)}

Low temperature thermoelectric materials are the only thermoelectric material used for the fabrication of fTEG due to the limiting temperature range of the supporting or filling substrates ${ }^{15,16}$. This is also a limiting factor in the performance of fTEG over conventional rigid thermoelectric devices. Usually fTEG's are fabricated by printing thermoelectric material over a flexible substrate or surrounding flexible support over small rigid thermoelectric materials ${ }^{13,15-17}$. Among these two methods printing on flexible substrates are the more reliable and cost effective method for the manufacturing of large area flexible thermoelectric generators. 
Among different fabrication methods for thermoelectric materials, wet deposition of nanocrystal- based colloidal inks using screen printing, inkjet printing, direct writing or other layer-by layer methods hold many advantages due to manufacturing flexibility and cost effectiveness. ${ }^{18,19}$ Using inkjet printers or dispensers several research groups have achieved a ZT of $\sim 0.3$ in thermoelectric films printed on flexible substrates ${ }^{13,20}$. Screen printing has also been explored as an efficient way to fabricate large areas and low cost thermoelectric devices ${ }^{16,21-24}$. Despite the above proof of concept demonstrations flexible thermoelectric films fabricated by printing methods continue to exhibit low ZT in the $0.1-0.3$ range, significantly lower than their rigid bulk counterparts fabricated using conventional approaches such as hot press or spark plasma sintering ${ }^{11}$. However, obtaining flexible thermoelectric materials with high figure of merit ZT through printing is an exacting challenge due to the difficulties in synthesizing high-performance thermoelectric inks, poor density, and limitation in sintering by the substrate or filling materials along with low electrical conductivity and also the high contact resistance of the printed films. ${ }^{25}$.

\subsection{Screen Printing}

The focus of this research is the development of high performing flexible thermoelectric devices using screen printing processes. Scalability, cost effectiveness and a wide range of viscosity for the paste along with higher thermoelectric powder loading allows screen printing to be a more favorable printing method ${ }^{16,19}$. The screen-printing technique consists of taking a special screen, designed for printing electrical conductors onto printed circuit boards. The screen has a metal or plastic wire mesh where the paste flows through it. The screen is generally placed about $1 \mathrm{~mm}$ from the substrate while the 
paste is then placed on top of the screen. A squeegee then goes across the surface of the screen, forcing the screen down onto the substrate and pushing the paste onto the substrate as shown in Figure 1.5. Ideal viscosities for screen-printing are in the range of 1-10 Pa-s (1000-10000 cP) and produce a thickness of 10-30 $\mu \mathrm{m}$ depending on the density of the mesh, as well as the emulsion thickness of the screen ${ }^{25}$.

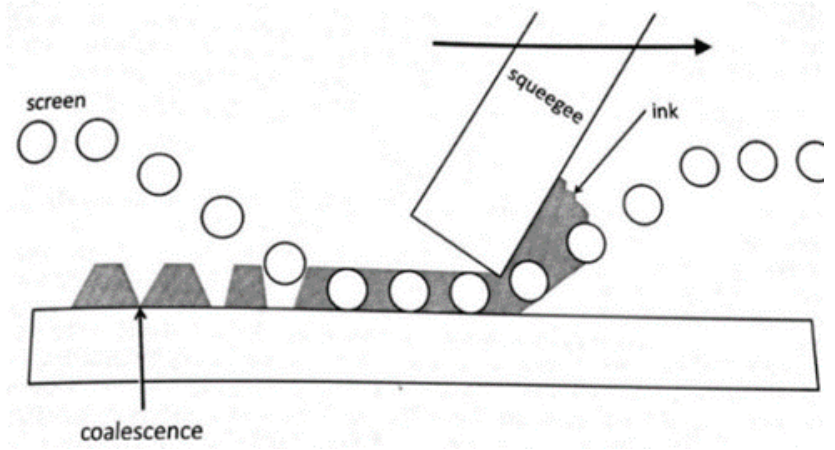

Figure 1.5 Visual example of how screen printers distribute inks onto substrates ${ }^{27}$

\subsection{Ink formulation}

A printable thermoelectric ink comprises of thermoelectric material with a capping agent, binder, solvents and property enhancing additives. Thermoelectric materials are prone to oxidation during Ink synthesis to avoid this either the materials are capped while a synthesis ${ }^{26}$ or encapsulating agent is added while making the ink which also acts as a dispersing agent for the nanomaterials. Polymer binders modify the rheological properties of the ink in order to achieve thixotropic properties while the solvent helps to adjust the viscosity of the entire paste. These thixotropic properties allow the ink to flow through the screen and create a high-resolution print with a uniform surface once the screen is removed ${ }^{27}$. Two types of binders and solvents are used for thermoelectric ink formulation one uses conductive binders which stay with the film even after sintering and the other utilizes nonconductive ink which completely burns of during annealing process. Other additives in the ink composition involves material for improving 
the adhesion of the printed films to the flexible substrates such as glass frits. ${ }^{16,21}$ Materials to engineer the carrier concentration and electron mobility of the printed films include copper and silver nanoparticles and also low melting temperature materials like tellurium nanoparticles ${ }^{28,29}$ for processes like liquid phase sintering.

Selection of binders and solvents are done in such a way to maximize the thermoelectric materials and minimize binders and solvents in every composition. This also depends upon the surface properties of the thermoelectric materials and its capping agents. The selection of a binder-solvent combination is critical to the success of printing thermoelectric materials. ${ }^{15}$ Solvent choice is equally as critical in the ink forming process. Beyond their compatibility with the binders, solvents must also meet certain criteria with respect to surface tension and boiling point. Solvents for screen-printing should have a low surface tension so that the print can have strong adhesion to the substrate. A surface tension value below 40 dynes/cm is acceptable for screen-printing, and surfactants are regularly employed in ink formulations to help with this cause. ${ }^{30}$ Boiling point values cannot be too low or the ink will evaporate too rapidly during the printing process and cause issues with sticking and low resolution prints. Too high of a boiling point will result in an ink that will have difficulty drying, and thus, a boiling point between 170 $200^{\circ} \mathrm{C}$ is a good range for screen printing thermoelectric inks. The printability of the film is determined by the particle size of the materials and viscosity of the paste. When designing an Ink for screen printing attention must be given to finely control the rheology of the ink or how the ink will respond to shear rate and different forces. A typical screen printing ink comes under a pseudo plastic behavior but it also act as thixotropic (time dependent relationship with shear stress $)^{30}$. Another important parameter to be considered 
is the wettability of the ink on the substrate, a better print on the substrate is achieved by controlling the surface tension of the ink and keeping it lower than the surface energy of the substrate surface.

Madan et al reported using a conductive adhesive for the formation of thermoelectric ink. Bisphenol f epoxy resin and an anhydride-based hardener with a catalyst butyl glycidyl ether. They were able to achieve a peak ZT of 0.31 with bismuth antimony telluride (BST) and this value is considerably lower than the peak ZT for rigid material. One of the main reasons is the restriction in annealing temperature due to the presence of conductive epoxy whose boiling temperature is close to $350^{\circ} \mathrm{C}$, which restricts the annealing temperature to a maximum of $350^{\circ} \mathrm{C}$. The conductivity of the epoxy is poor ${ }^{13}$, this brings down the $\mathrm{ZT}$ value of the material. In another study inorganic thermoelectric material is combined with organic thermoelectric material PEDOT: PSS and poly (acrylic acid) PAA to make the printed films with several micrometer thicknesses. In order to connect the BST particle electrically and mechanically, but due to limiting annealing temperature and poor density the maximum ZT achieved is only up to 0.2 , even it expressed superior flexibility for the films. ${ }^{31}$

A second approach involves using a nonconductive polymer as a binder which acts as a capping agent as well as a binder ${ }^{32}$, thermoelectric powder suspended in a polystyrene polymer diluted in toluene. Complete removal of the binder present in the mixture happens at $200^{\circ} \mathrm{C}$, after burning of the binder from the films which leads a residue in the printed films which either creates additional porosity or acts as an insulating material and eventually brings down the electrical conductivity of the films. There have been studies on incorporating property enhancing additives, additional items 
that can be added to ink formulations include dispersants, glass sintering aids and metal nanoparticles. Kim et al used small glass frits along with the binder solvent mixture in order to enhance the bonding strength of the printed pellets prepared using screen printing. ${ }^{21}$ The Talapin group introduced a nanocrystal glue approach in order to enhance the density of the thermoelectric film. ${ }^{33}$ In this approach a nanocrystal capped with chanogenido-metallate ligands are introduced in the thermoelectric matrix, these metal ligands will deposit at the grain boundaries and will act as a solder material to improve connectivity of the thermoelectric materials and reach a peak power factor of $1.96 \mathrm{mWm}^{-}$ ${ }^{1} \mathrm{~K}^{-2}$. Even though this is a good approach that involves sophisticated chemical processes and the inks prepared are in colloidal form which is not suitable for screen printing approach.

\subsection{Aerosol Jet Printing}

For additive printing of the TEG, an Aerosol Jet Printer (Optomec200) was used, as compared to contemporary printing techniques such as screen printing ${ }^{16,34}$, Inkjet printing $^{20,35}$, spin coating ${ }^{36}$ and dispenser printing ${ }^{37}$ Aerosol jet printing offers distinct advantages in terms of the possibility of using inks with a very wide range of viscosities $(1-1000 \mathrm{cP})$ and also provides more fine printing parameters ${ }^{38}$.

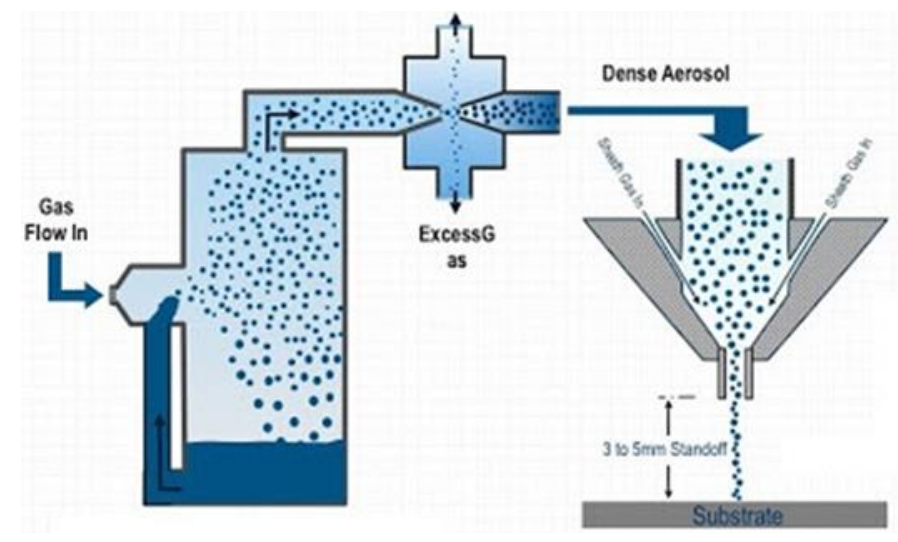

Figure 1.6 Schematic representation of Aerosol Jet Printing @ Optomec 
Figure 1.6 a representation of the Aerosol Jet Printer (AJP), shows the ink was atomized (aerosol) using a pneumatic atomizer; high pressure gas is injected into the chamber to create a capillary action assisted spraying of the inks. Pneumatic atomizers can magnetically stir the ink mixture allowing for better atomization of the ink having non -homogeneously suspended particles in the dispersion medium ${ }^{38}$. The atomized gases are protected by a sheath of $\mathrm{N}_{2}$ gas creating a clog resistant nozzle and high density micro droplets. The continuous stream of inks helps to tightly focus the jetting to the substrate creating line features as small as $10 \mu \mathrm{m}$ in width and few hundred nanometers to micrometer thickness. AJP allows a large viscosity range for the colloidal inks varying from $1 \mathrm{cP}$ to $1000 \mathrm{cP}$. Recently ${ }^{39} \mathrm{Calin}$ Ou et al reported printing a MWCNT, $\mathrm{Sb}_{2} \mathrm{Te}_{3}$ and PEDOT:PSS composite ink on to a flexible substrate. Flexible thermoelectric legs manufactured a best power factor of $41 \mu \mathrm{V} / \mathrm{mK}^{2}$ with superior flexible properties up to $36 \mathrm{k}$ continuous flexing cycles. This shows AJP is a substantial tool in developing highly flexible thermoelectric films with a fine feature size.

\subsection{Intense Pulse Light Sintering}

Conventional thermal sintering of thermoelectric materials requires high temperature sintering for a long time to achieve the best TE properties. Flexible thermoelectric generators are limited to low temperature thermal sintering due to low melting temperature of the polymer substrates. In this research an intense flash of UV light from a Xenon lamp to sinter TE nanoparticles within milliseconds under ambient conditions were explored. Intense pulse light (IPL) sintering or photonic sintering utilizes a Xenon lamp capable of emitting light 200-800nm with high intensities for a short period of time. ${ }^{39}$ Sudden bursts of energy will allow the nanocrystals to raise their 
temperature without increasing the temperature of the substrate. This sudden increase in temperature will help to sinter the thermoelectric nanocrystals because of the low melting temperature of the nanomaterials. The amount of energy deposited can be controlled by the energy of the pulse and the duration at which the material is exposed to the light source as shown in Figure 1.7. Danaei et al performed a COMSOL modeling and experiment analysis of $\mathrm{Bi}_{2} \mathrm{Se}_{0.3} \mathrm{Te}_{2.7}$ nanocrystals with different duration of the pulse intensities. It is observed that with an increase in energy duration from $0 \mathrm{~ms}$ to $2 \mathrm{~ms}$ a $16 \%$ reduction in porosity is observed and a corresponding improvement in the electrical conductivity of the deposited films. This shows that high energy pulse light sintering is an efficient technique for the sintering of thermoelectric materials without destroying the polymers.

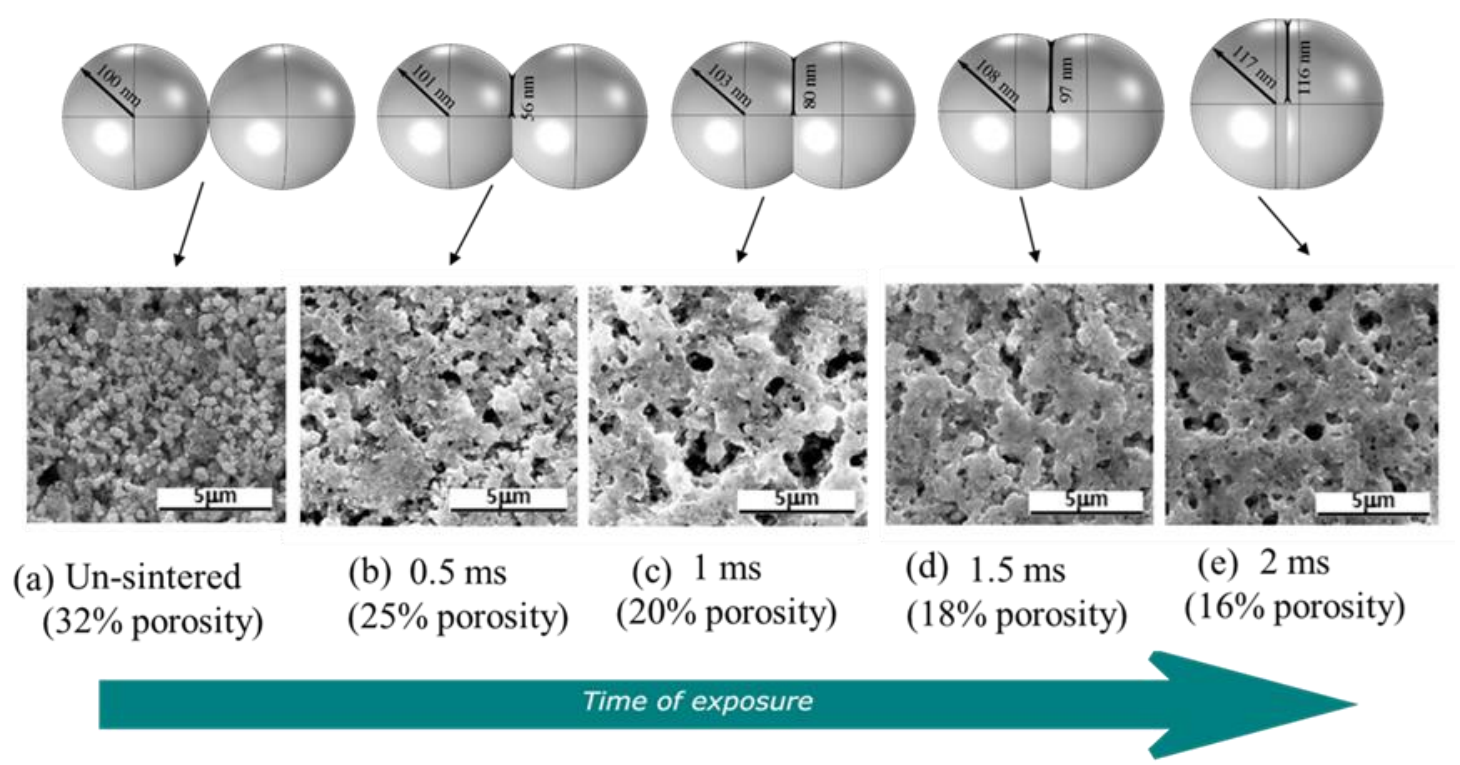

Figure 1.7 (a-e) Particle coalescence calculated by COMSOL modeling and comparing to the corresponding SEM images of the samples. ${ }^{39}$ 


\subsection{Conclusion}

1. To obtain a successful printing, printable thermoelectric inks have to be synthesized. Printed thermoelectric inks consists of thermoelectric particles, performance enhancing additives, binders and solvents.

2. Thermoelectric inks are designed to maximize the TE nanoparticles content with minimum solvent and binders to enhance the density and reduce porosity.

3. A detailed study is required for enhancing the quality of films optimizing printing parameters, drying and cold compaction.

4. Systematic thermal studies are necessary to optimize the sintering conditions, varying performing carrier gas, elemental vapor pressures and hot pressing

5. Performance enhancing additives help to improve the thermoelectric properties and mechanical properties of fTEG's

6. Aerosol jet printing and photonic sintering techniques are great tools for roll to roll manufacturing of high array density flexible films.

\subsection{References}

1 Han, C., Sun, Q., Li, Z. \& Dou, S. X. Thermoelectric Enhancement of Different Kinds of Metal Chalcogenides. Advanced Energy Materials 6, 1600498, doi:10.1002/aenm.201600498 (2016).

2 Madan, D., Wang, Z., Wright, P. K. \& Evans, J. W. Printed flexible thermoelectric generators for use on low levels of waste heat. Applied Energy 156, 587-592, doi:10.1016/j.apenergy.2015.07.066 (2015).

3 Fan, F. R., Tang, W. \& Wang, Z. L. Flexible Nanogenerators for Energy Harvesting and Self-Powered Electronics. Adv Mater 28, 4283-4305, doi:10.1002/adma.201504299 (2016). 
4 Choi, K. H., Yoo, J., Lee, C. K. \& Lee, S. Y. All-inkjet-printed, solid-state flexible supercapacitors on paper. Energ Environ Sci 9, 2812-2821, doi:10.1039/c6ee00966b (2016).

5 Oh, J. Y. et al. Chemically exfoliated transition metal dichalcogenide nanosheetbased wearable thermoelectric generators. Energ Environ Sci 9, 1696-1705, doi:10.1039/c5ee03813h (2016).

6 Gao, M., Li, L. H., Li, W. B., Zhou, H. H. \& Song, Y. L. Direct Writing of Patterned, Lead-Free Nanowire Aligned Flexible Piezoelectric Device. Adv Sci 3, doi:Artn 160012010.1002/Advs.201600120 (2016).

7 Ye, F. et al. Soft-cover deposition of scaling-up uniform perovskite thin films for high cost-performance solar cells. Energ Environ Sci 9, 2295-2301, doi:10.1039/c6ee01411a (2016).

8 Dresselhaus, M. S. et al. New directions for low-dimensional thermoelectric materials. Adv Mater 19, 1043-1053, doi:10.1002/adma.200600527 (2007).

9 Hicks, L. D. \& Dresselhaus, M. S. Effect of Quantum-Well Structures on the Thermoelectric Figure of Merit. Phys Rev B 47, 12727-12731, doi:DOI 10.1103/PhysRevB.47.12727 (1993).

10 Snyder, G. J. \& Toberer, E. S. Complex thermoelectric materials. Nat Mater 7, 105-114, doi:10.1038/nmat2090 (2008).

11 Bahk, J. H., Fang, H. Y., Yazawa, K. \& Shakouri, A. Flexible thermoelectric materials and device optimization for wearable energy harvesting. J Mater Chem C 3, 10362-10374, doi:10.1039/c5tc01644d (2015).

12 Rull-Bravo, M., Moure, A., Fernández, J. F. \& Martín-González, M. Skutterudites as thermoelectric materials: revisited. RSC Advances 5, 41653-41667, doi:10.1039/C5RA03942H (2015).

13 Madan, D. et al. Enhanced Performance of Dispenser Printed MA n-type Bi2Te3 Composite Thermoelectric Generators. Acs Appl Mater Inter 4, 6117-6124, doi:10.1021/am301759a (2012). 
14 Bottner, H. et al. New thermoelectric components using microsystem technologies. Journal of Microelectromechanical Systems 13, 414-420, doi:10.1109/JMEMS.2004.828740 (2004).

15 Varghese, T. et al. High-performance and flexible thermoelectric films by screen printing solution-processed nanoplate crystals. Sci Rep-Uk 6, doi:ARTN $3313510.1038 /$ srep33135 (2016).

16 Kim, S. J., We, J. H. \& Cho, B. J. A wearable thermoelectric generator fabricated on a glass fabric. Energ Environ Sci 7, 1959-1965, doi:10.1039/c4ee00242c (2014).

17 Hyland, M., Hunter, H., Liu, J., Veety, E. \& Vashaee, D. Wearable thermoelectric generators for human body heat harvesting. Applied Energy 182, 518-524, doi:10.1016/j.apenergy.2016.08.150 (2016).

18 Jiao, F. et al. Inkjet-printed flexible organic thin-film thermoelectric devices based on p- and n-type poly(metal 1,1,2,2-ethenetetrathiolate)s/polymer composites through ball-milling. Philos T R Soc A 372, doi:Artn 2013000810.1098/Rsta.2013.0008 (2014).

19 Koplow, M., Chen, A. \& Steingart, D. Thick film thermoelectric energy harvesting systems for biomedicalapplications, Medical Devices and Biosensors. (2008).

$20 \mathrm{Lu}, \mathrm{Z}$. Y. et al. Fabrication of Flexible Thermoelectric Thin Film Devices by Inkjet Printing. Small 10, 3551-3554, doi:10.1002/smll.201303126 (2014).

21 Kim, S. J., We, J. H., Kim, J. S., Kim, G. S. \& Cho, B. J. Thermoelectric properties of P-type $\mathrm{Sb} 2 \mathrm{Te} 3$ thick film processed by a screen-printing technique and a subsequent annealing process. J Alloy Compd 582, 177-180, doi:10.1016/j.jallcom.2013.07.195 (2014).

22 Lee, H. B. et al. Thin-Film Thermoelectric Module for Power Generator Applications Using a Screen-Printing Method. J Electron Mater 40, 615-619, doi:10.1007/s11664-010-1481-0 (2011). 
23 We, J. H., Kim, S. J., Kim, G. S. \& Cho, B. J. Improvement of thermoelectric properties of screen-printed Bi2Te3 thick film by optimization of the annealing process. J Alloy Compd 552, 107-110, doi:10.1016/j.jallcom.2012.10.085 (2013).

24 Kamyshny, A. \& Magdassi, S. Conductive Nanomaterials for Printed Electronics. Small 10, 3515-3535, doi:10.1002/smll.201303000 (2014).

25 Dorey, R. A. Ceramic Thick films for MEMS and Microdevices. (2011).

26 Mehta, R. J. et al. A new class of doped nanobulk high-figure-of-merit thermoelectrics by scalable bottom-up assembly. Nat Mater 11, 233-240, doi:10.1038/Nmat3213 (2012).

27 Stevenson, R. Flexible thermoelectric generators for biomedical applications Master of science thesis, boise state university.

28 Lee, K. H. et al. Enhancement of Thermoelectric Figure of Merit for Bi0.5Sb1.5Te3 by Metal Nanoparticle Decoration. J Electron Mater 41, 11651169, doi:10.1007/s11664-012-1913-0 (2012).

29 Il Kim, S. et al. Dense dislocation arrays embedded in grain boundaries for highperformance bulk thermoelectrics. Science 348, 109-114, doi:10.1126/science.aaa4166 (2015).

30 Gilleo, K. Rheology and surface chemistry for screen printing.

31 Kato, K., Hagino, H. \& Miyazaki, K. Fabrication of Bismuth Telluride Thermoelectric Films Containing Conductive Polymers Using a Printing Method. J Electron Mater 42, 1313-1318, doi:10.1007/s11664-012-2420-z (2013).

32 Navone, C., Soulier, M., Plissonnier, M. \& Seiler, A. L. Development of $(\mathrm{Bi}, \mathrm{Sb})(2)(\mathrm{Te}, \mathrm{Se})(3)-$ Based Thermoelectric Modules by a Screen-Printing Process. J Electron Mater 39, 1755-1759, doi:10.1007/s11664-010-1187-3 (2010).

33 Son, J. S. et al. All-Inorganic Nanocrystals as a Glue for BiSbTe Grains: Design of Interfaces in Mesostructured Thermoelectric Materials. Angew Chem Int Edit 53, 7466-7470, doi:10.1002/anie.201402026 (2014). 
34 Wei, Q., Mukaida, M., Kirihara, K., Naitoh, Y. \& Ishida, T. Polymer thermoelectric modules screen-printed on paper. RSC Advances 4, 28802-28806, doi:10.1039/C4RA04946B (2014).

35 Bubnova, O. et al. Optimization of the thermoelectric figure of merit in the conducting polymer poly(3,4-ethylenedioxythiophene). Nat Mater 10, 429, doi:10.1038/nmat3012 (2011).

36 Russ, B., Glaudell, A., Urban, J. J., Chabinyc, M. L. \& Segalman, R. A. Organic thermoelectric materials for energy harvesting and temperature control. Nature Reviews Materials 1, 16050, doi:10.1038/natrevmats.2016.50 (2016).

37 Chen, A., Madan, D., Wright, P. K. \& Evans, J. W. Dispenser-printed planar thick-film thermoelectric energy generators. Journal of Micromechanics and Microengineering 21, 104006, doi:10.1088/0960-1317/21/10/104006 (2011).

38 Smith, M., Choi, Y. S., Boughey, C. \& Kar-Narayan, S. Controlling and assessing the quality of aerosol jet printed features for large area and flexible electronics. Flexible and Printed Electronics 2, 015004, doi:10.1088/2058-8585/aa5af9 (2017).

39 Ou, C. et al. Enhanced thermoelectric properties of flexible aerosol-jet printed carbon nanotube-based nanocomposites. APL Materials 6, 096101, doi:10.1063/1.5043547 (2018).

40 Danaei, R. et al. Ultrafast Fabrication of Thermoelectric Films by Pulsed Light Sintering of Colloidal Nanoparticles on Flexible and Rigid Substrates. Advanced Engineering Materials 21, 1800800, doi:10.1002/adem.201800800 (2019). 


\title{
CHAPTER TWO: HIGH-PERFORMANCE AND FLEXIBLE THERMOELECTRIC FILMS BY SCREEN PRINTING SOLUTION-PROCESSED NANOPLATE CRYSTALS
}

This chapter is published by Nature Research in Scientific Reports and should be referenced appropriately

\author{
Reference \\ Varghese T, Hollar C, Richardson J, Kempf N, Han C, Gamarachchi P, et al. \\ High-performance and flexible thermoelectric films by screen printing solution-processed \\ nanoplate crystals. Scientific Reports. 2016;6:33135
}

Reproduced/modified by permission of the Nature research

*This chapter includes modifications from the originally published version. 


\title{
HIGH-PERFORMANCE AND FLEXIBLE THERMOELECTRIC FILMS BY SCREEN PRINTING SOLUTION-PROCESSED NANOPLATE CRYSTALS
}

\author{
Tony Varghese ${ }^{1}$ \\ Courtney Hollar ${ }^{2}$ \\ Nicholas Kempf ${ }^{2}$ \\ Chao $\operatorname{Han}^{2}$ \\ David Estrada ${ }^{1}$ \\ Rutvik J. Mehta ${ }^{3}$ \\ Yanliang Zhang ${ }^{1,2}$
}

${ }^{1}$ Department of Materials Science and Engineering, Boise State University, Boise, ID 83725, United States 
${ }^{2}$ Department of Mechanical and Biomedical Engineering, Boise State University, Boise, ID 83725, United States

${ }^{3}$ ThermoAura, inc. 132 B. Railroad Avenue, Colonie, NY 12205, United States

\subsection{Abstract}

Screen printing allows for direct conversion of thermoelectric nanocrystals into flexible microscale energy converters or coolers. However, obtaining flexible thermoelectric materials with high figure of merit ZT through printing is an exacting challenge due to the difficulties to synthesize high-performance thermoelectric inks and the poor density and electrical conductivity of the printed films. Here, we demonstrate high ZT flexible films by screen printing bismuth telluride based nanocrystal inks synthesized using a high-yield and low-cost microwave-stimulated wet-chemical method. Thermoelectric films of several tens of microns thicknesses were screen printed onto a flexible polyimide substrate followed by cold compaction and pressureless sintering. The n-type films demonstrate a peak ZT of 0.43 along with superior flexibility, which is among the highest reported ZT values in flexible thermoelectric materials. The highly scalable and cost-effective process to fabricate flexible thermoelectric materials demonstrated here opens up many opportunities to transform thermoelectric energy harvesting and cooling applications.

Keywords: Thermoelectric films, flexibility, screen printing, nanoplates 


\subsection{Introduction}

Thermoelectric conversion is a solid-state and environmental-friendly energy conversion technology with broad applications including solid-state cooling, energy harvesting, and waste heat recovery ${ }^{1}$. Flexible thermoelectric devices are especially attractive for waste heat recovery along contoured surfaces and for energy harvesting applications to power sensors, biomedical devices, and wearable electronics - an area under exponential growth ${ }^{2}$.

The efficiency of thermoelectric materials is determined by the figure of merit ZT defined as ZT $=\alpha^{2} \sigma T / \kappa$, where $\alpha, \sigma, \kappa$ and $T$ are the Seebeck coefficient, electrical conductivity, thermal conductivity and absolute temperature respectively ${ }^{3,4}$. Nanostructured thermoelectric materials have been widely studied in recent years and proven to have unique and superior thermoelectric performance compared to their bulk counterparts due to the ability to tailor electron and phonon transport and effectively increase $\mathrm{ZT}^{5-7}$. Despite significant ZT improvements in nanostructured materials ${ }^{8-10}$, the lack of scalable and low-cost manufacturing processes remains a major challenge to the wide use of these materials ${ }^{11}$. In addition, major progress in ZT enhancement through nanostructuring has historically been achieved in mechanically rigid materials, while flexible thermoelectric materials are still relatively unexplored and have fairly low $\mathrm{ZT}^{2}$.

Among all the methods to fabricate thermoelectric materials, wet-deposition of nanocrystal based colloidal inks using screen printing, inkjet printing, direct writing or other layer by layer methods hold many advantages due to the ability to directly convert nanocrystal inks into micro/macroscale functional materials and devices with great scalability, flexibility and cost effectiveness ${ }^{12,13}$. Using inkjet or disperser printing, 
several research groups have achieved ZT of $\sim 0.3$ in thermoelectric films printed on flexible substrates ${ }^{14,15}$. Screen printing has also been explored as a more efficient way to fabricate thermoelectric devices ${ }^{16-19}$. Despite the above proof-of-concept demonstrations, flexible thermoelectric films fabricated by printing methods continue to exhibit fairly low ZT in 0.1-0.3 range, significantly lower than their rigid bulk counterparts fabricated using conventional approaches such as hot press or spark plasma sintering ${ }^{2}$. There are many challenges in printing efficient and flexible thermoelectric materials using nanocrystals, including scalable synthesis of high-performance nanocrystals, nanocrystal surface oxidation during printing processes, and poor density and electrical conductivity of the printed films ${ }^{20}$. Thermoelectric films, including nanocrystal synthesis, nano-ink processing, screen printing of thermoelectric films on flexible substrate, and sintered flexible films.

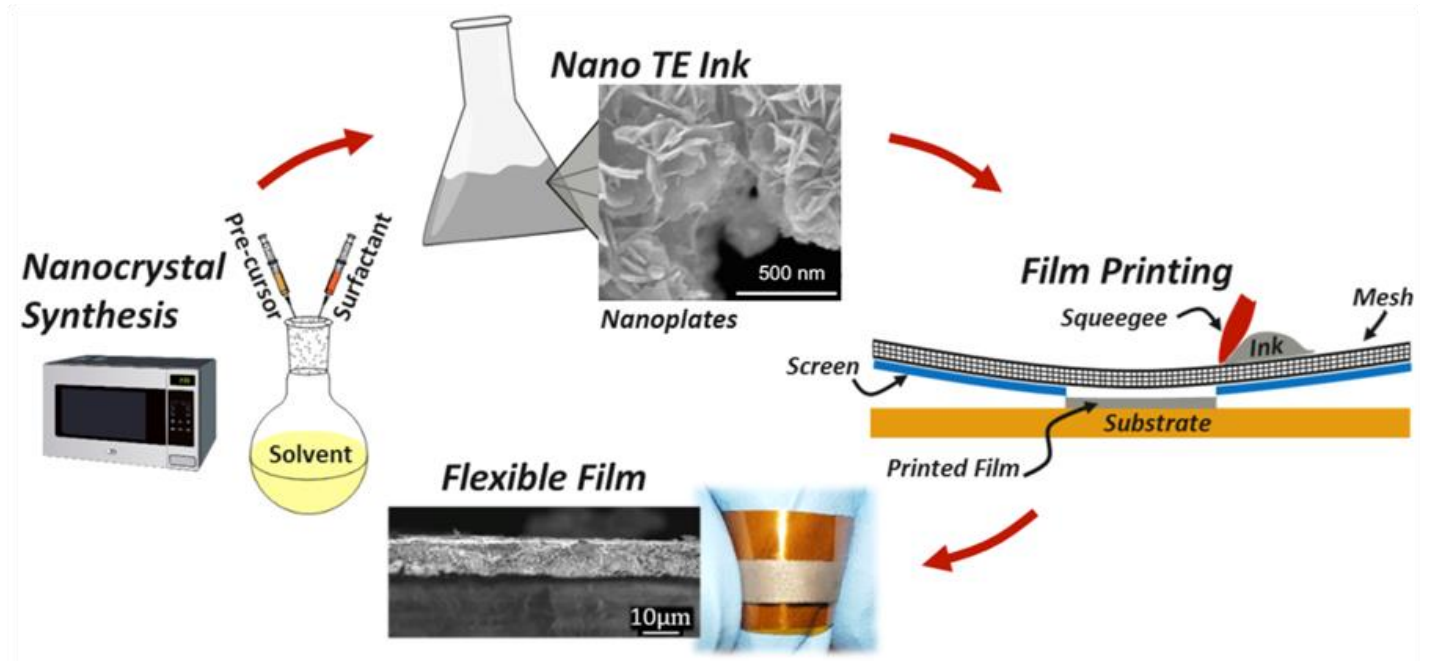

Figure 2.1 Schematic illustration of overall fabrication process for the flexible

Here, we report a study of flexible thermoelectric films by screen printing colloidal inks composed of bismuth telluride based nanoplates fabricated using a scalable microwave-stimulated wet chemical approach ${ }^{21}$ (shown in Figure 1). The peak ZT of 
our flexible films reached 0.43 at $175^{\circ} \mathrm{C}$ due to a combination of high power factor and low thermal conductivity, which is among the highest ZT reported for flexible thermoelectric materials fabricated by printing. The films demonstrate superior flexibility with negligible changes in electric resistance within 150 bending cycles. In addition to the unprecedented high ZT and flexibility, another significant advantage of our work is the use of thioglycolic acid (TGA) as a surface capping agent to inhibit nanocrystal oxidation $^{21}$, thus enabling large-scale manufacturing at ambient conditions.

\subsection{Methods}

\section{$\underline{2.3 .1 \quad \text { Nanocrystal Ink Synthesis }}$}

Our doped and functionalized pnictogen chalcogenide nanocrystals of $\mathrm{Bi}_{2} \mathrm{Te}_{2.8} \mathrm{Se}_{0.2}$ were synthesized using a microwave stimulated wet-chemical synthesis method based on inexpensive organic solvents and metal salts described earlier ${ }^{21}$. In this method, the reaction between molecularly ligated chalcogen and pnictogen complexes was activated by microwave stimulation with the presence of thioglycolic acid (TGA), which serves as a shape-directing, oxide-inhibiting and sulfur-dopant delivery agent. The resulting precipitate is cleaned and dried in ambient conditions to obtain powders consisting of single-crystal nanoplates of 5- to 20-nm-thickness with bounding edge dimensions ranging between 50 to $500 \mathrm{~nm}$. The prepared thioglycolic acid (TGA) coated BiSeTe nanopowders (58 wt.\%), 1 wt.\% Glass Frits (from Artglass Supplies, 325 mesh), 2 wt.\% Disperbyk-110 (Binder, from BYK U.S.A. Inc.) and 39 wt.\% $\alpha$-Terpineol (Solvent, 90\% purity, from Sigma-Aldrich) are weighted and mixed by vortex mixing for $10 \mathrm{~min}$, then the mixture is vibrated on a Vibrator for $5 \mathrm{~min}$ to form a homogeneous paste. This recipe is then optimized to get reasonable viscosities for screen printing. The 
mix ratio of solvent and binder did not affect the film properties as they are evaporated during the drying process. The usage of glass frits is to increase adhesion of the film with Kapton film as the glass frits (mainly made of $\mathrm{Na}, \mathrm{Al}, \mathrm{Si}, \mathrm{O}, \mathrm{K}, \mathrm{Ca}$ ) has a melting point of lower than $400{ }^{\circ} \mathrm{C}$. However, as the glass frits possess pretty low electrical conductivity, we fixed it to $1 \mathrm{wt} . \%$ to get both high electrical conductivity and good adhesion with Kapton substrate.

\subsubsection{Flexible Film Fabrication}

As prepared ink is screen printed on flexible polyimide substrates, the thermoelectric films of various thicknesses in the range of 10-100 $\mu \mathrm{m}$ were obtained by controlling the screen meshes and the number of repeated print passes. The printed films were first dried in air at $200{ }^{\circ} \mathrm{C}$ on a hot plate to remove the solvent and binder, followed by a cold compaction using a hydraulic press to consolidate the films. The film was finally sintered at $430{ }^{\circ} \mathrm{C}$ for 45 minutes in vacuum in order to remove the TGA surfactant and further improve the film density. The sintering temperatures are kept below the melting point of the polyimide substrate, though better thermoelectric properties could be obtained at higher sintering temperatures.

\subsubsection{Thermoelectric Property Measurement}

The temperature-dependent in-plane electrical conductivity and Seebeck coefficient of the film sample were measured simultaneously using a commercial Linseis Seebeck and resistivity instrument. The above two properties of the same sample were also measured using a home-built testing system, and the two sets of measurement results are within $2 \%$. In order to measure the thermal conductivity of the sample, a freestanding film of about $100 \mu \mathrm{m}$ thickness is prepared under the same conditions as those for 
preparing thinner films on substrate, and these films show nearly identical thermoelectric properties independent of their thicknesses. The temperature-dependent cross-plane thermal diffusivity of the freestanding film was measured using a laser flash instrument. The cross-plane thermal conductivity is then determined using the sample density measured using Archimedes method and the specific heat measured using the DSC instrument. The in-plane thermal conductivity of the freestanding film was measured directly using a steady-state method in vacuum, which is within $5 \%$ of the cross-plane thermal conductivity, indicating the sample is isotropic.

The thickness of the film is around $10 \mu \mathrm{m}$ while the control pellet sample is 100 $\mu \mathrm{m}$ and $500 \mu \mathrm{m}$, respectively. To fully understand the electrical conductivity differences on film and pellet samples, Hall measurement under room temperature is conducted in a Physics Property Measurement System (PPMS) with a magnetic field range from -1 T to $1 \mathrm{~T}$. The pellet and film are four wire connected to the sample holder as shown in Figure 2.2. Relevant calculations are listed below:

$$
\begin{gathered}
R_{\text {Hall }}=\frac{V_{\text {Hall }}}{I} \\
R_{H}=\frac{R_{\text {Hall }} \times T}{B} \times 10^{8} \mathrm{~cm}^{3} / C \\
n=-\frac{1}{R_{H} * e} \\
\mu=\sigma \times R_{H}
\end{gathered}
$$

$\mathrm{R}_{\text {Hall }}$ is the Hall resistance $(\Omega)$; $\mathrm{V}_{\text {Hall }}$ is the voltage between $\mathrm{V}+$ and $\mathrm{V}$-, or Hall voltage $(\mathrm{V})$; I is the excitation current $(0.4 \mathrm{~mA}$ for film and $2 \mathrm{~mA}$ for pellet $)$. $\mathrm{T}$ is the thickness of the sample $(\mathrm{cm})$; B is the magnetic field $(\mathrm{Oe}) ; \mathrm{R}_{\mathrm{H}}$ is Hall coefficient $\left(\mathrm{cm}^{3} / \mathrm{C}\right)$; 
$\mathrm{n}$ is the carrier concentration $\left(/ \mathrm{cm}^{3}\right)$; e is the charge of single electron $\left(1.6 \times 10^{-19} \mathrm{C}\right) ; \sigma$ is electrical conductivity of the sample (S/cm); $\mu$ is carrier mobility $\left[\mathrm{cm}^{2} /(\mathrm{V} . \mathrm{s})\right]$.

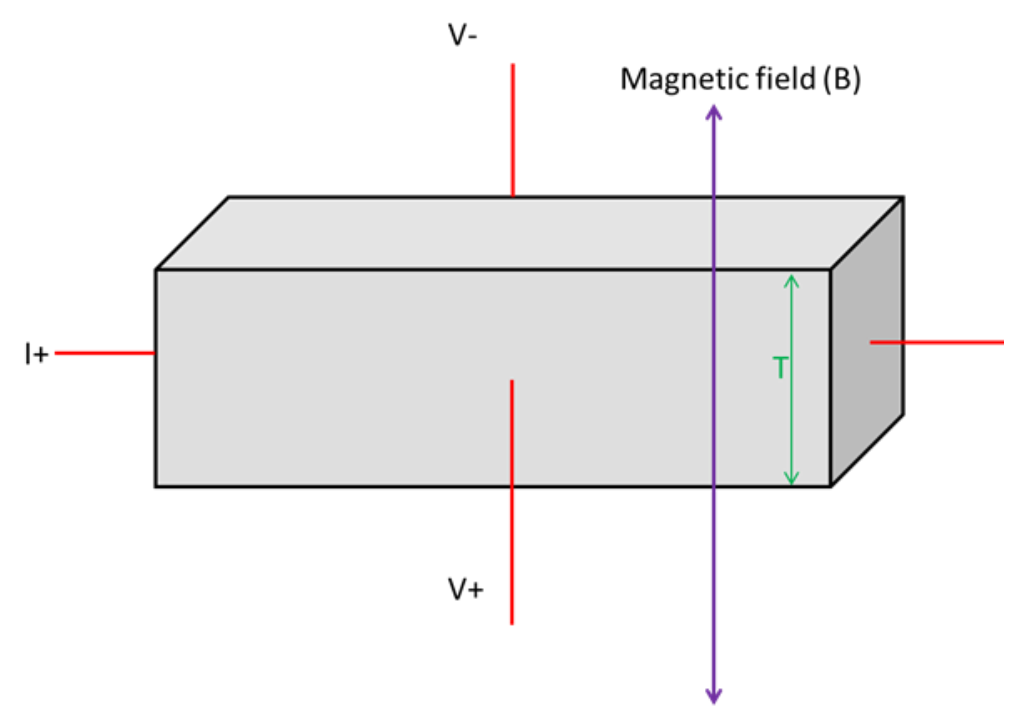

Figure 2. 2 Schematic of Hall measurement sample by Four Wire method.

The calculated Hall coefficient $\left(\mathrm{R}_{\mathrm{H}}\right)$, carrier concentration $(\mathrm{n})$, as well as carrier mobility $(\mu)$ is listed in Table 1 . The negative sign of $R_{H}$ indicated the $n$ type conductance. Carrier concentrations of these two samples are in the same magnitude and the film shows slightly higher carrier concentration while the carrier mobility of the pellet is obviously higher than in the film. Thereby the electrical conductivity of the flexible film is lower than that of the pellet sample mainly due to the lower electron mobility by impurities and porosity. 
Table 2.1 Hall measurement result of the BiSeTe film and pellet

\begin{tabular}{|c|c|c|c|c|c|}
\hline Sample & $\begin{array}{c}\text { Temperature } \\
{[\mathrm{K}]}\end{array}$ & $\begin{array}{c}\text { Hall } \\
\text { coefficient } \\
{\left[\mathrm{R}_{\mathrm{H}}, \mathrm{m}^{3} / \mathrm{C}\right]}\end{array}$ & $\begin{array}{c}\text { Carrier } \\
\text { concentration } \\
{\left[\mathrm{n}, / \mathrm{cm}^{3}\right]}\end{array}$ & $\begin{array}{c}\text { Electrical } \\
\text { mobility } \\
{\left[\mu, \mathrm{cm}^{2} /(\mathrm{V} . \mathrm{S})\right]}\end{array}$ & $\begin{array}{c}\text { conductivity } \\
{[\sigma, \mathrm{S} / \mathrm{cm}]}\end{array}$ \\
\hline Pellet & 305 & -0.44 & $1.42 \times 10^{19}$ & 290 & 660 \\
\hline Film & 305 & -0.41 & $1.56 \times 10^{19}$ & 127 & 310 \\
\hline
\end{tabular}

\subsection{Results and Discussion}
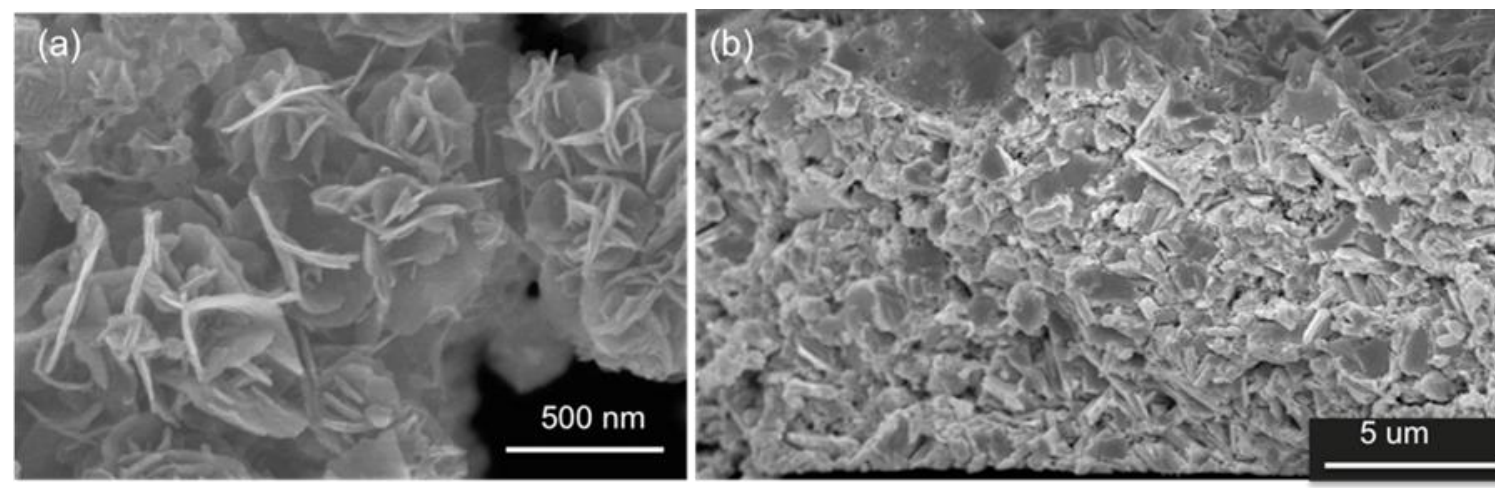

Figure 2.3 SEM images of (a) the $\mathrm{Bi}_{2} \mathrm{Te}_{2.8 \mathrm{Se}} \mathrm{S} .2$ nanocrystals and (b) the cross section of the flexible film.

Figure2. 3 (a) shows scanning electron microscope (SEM) images of the $\mathrm{Bi}_{2} \mathrm{Te}_{2.8} \mathrm{Se}_{0.2}$ nanocrystals, indicating the plate like structures of several tens of nanometers thickness. Figure 2.3 (b) shows a cross-section SEM image of a flexible $\mathrm{Bi}_{2} \mathrm{Te}_{2.8} \mathrm{Se}_{0.2}$ film of about $10 \mu \mathrm{m}$ thickness fabricated by screen printing. The films have about $85 \%$ relative density, and contain nanoscale pores primarily due to incomplete compaction of the nanocrystals and the removal of the additives in the ink. 


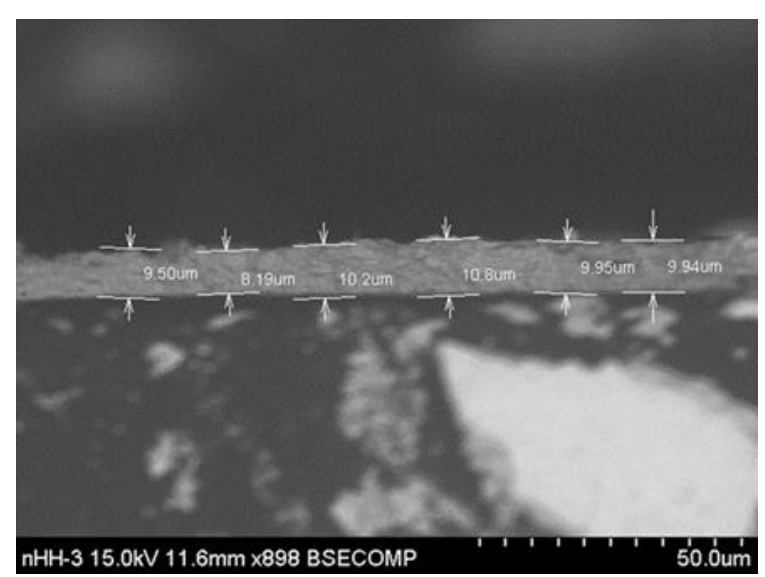

Figure 2. 4 Thickness measurement for film using SEM.

Figure 2.5 shows the temperature-dependent thermoelectric properties of a flexible film printed using the nanocrystal ink and a control pellet sample of $500 \mu \mathrm{m}$ thickness made by the pure nanocrystal powders using cold compaction and sintering under the same conditions. The relative densities of the film and the pellet are $85 \%$ and 90\% respectively. As shown in Figure 2.5(a), the room-temperature electrical conductivity of the film is about $56 \%$ lower than the pellet due to the impurities (dielectric glass additives) and higher porosity which result in lower electron motilities in the film. Figure 2.5 (b) shows the Seebeck coefficients of the two samples are within $10 \%$ for the entire measurement temperature, indicating approximately the same compositions and carrier concentrations for both the film and the pellet. 

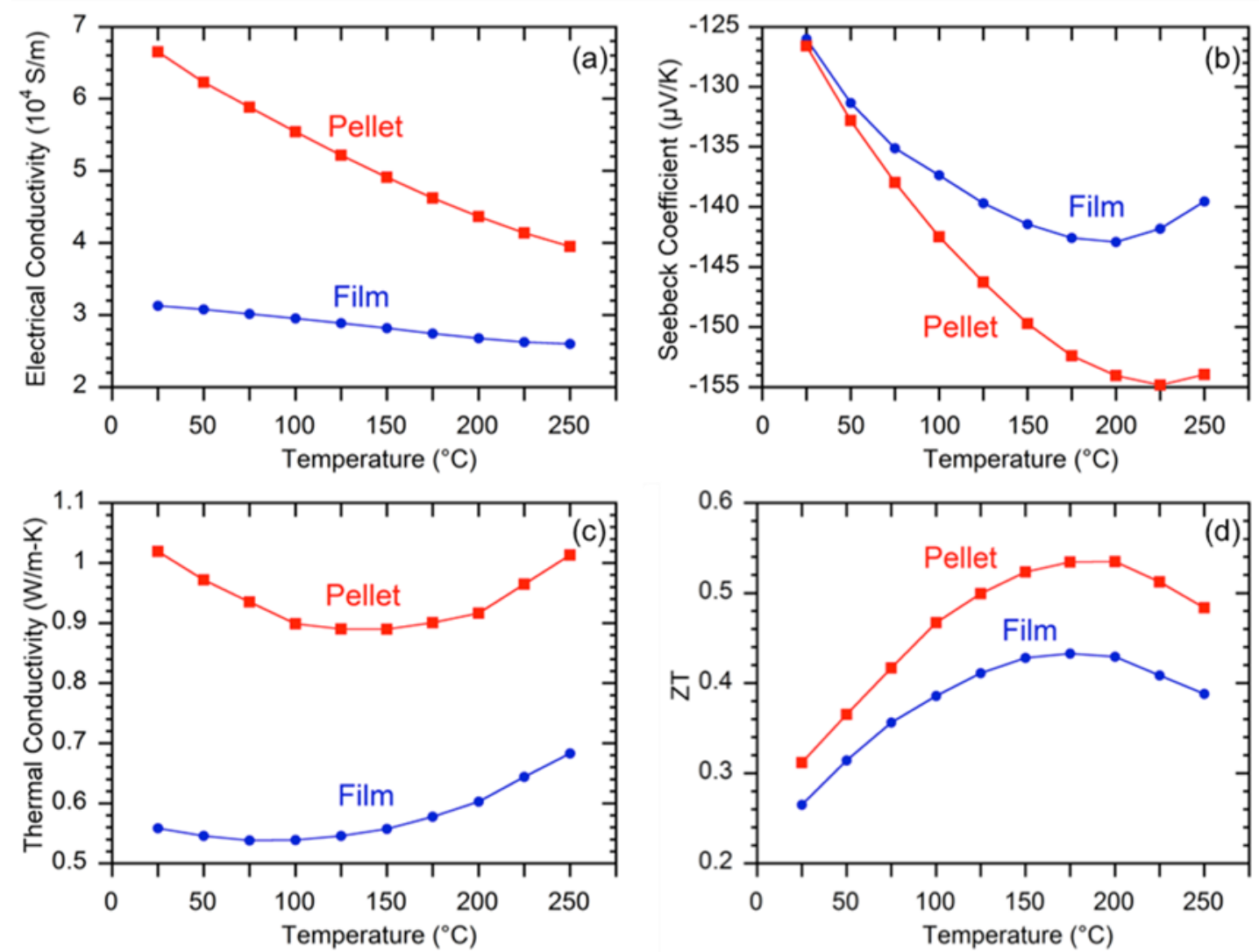

Figure 2.5 Temperature-dependent (a) Electrical conductivity (b) Seebeck coefficient (c) Thermal conductivity and (d) ZT of a flexible film and a reference pellet as a control pellet.

The in-plane thermal conductivity of the printed films was measured using the parallel thermal conductance method. Two separate measurements were conducted in order to determine the baseline thermal conductance of the test setup and total thermal conductance including the test setup and the sample, the difference of which yielded the sample thermal conductance. A freestanding film $(15 \mathrm{mmX} 4 \mathrm{~mm} \mathrm{X} 0.1 \mathrm{~mm})$ was prepared by removing the substrate after cold compaction and before sintering when the adhesion between the film and the substrate was still very weak. The film was then sintered at the same conditions as the films on substrate. The electrical conductivity and Seebeck coefficient of the $100 \mu \mathrm{m}$ thick freestanding film and the $10 \mu \mathrm{m}$ thick film 
shown in Figure 2.5 are within 6\%, indicating nearly identical thermoelectric properties independent of the sample thicknesses.
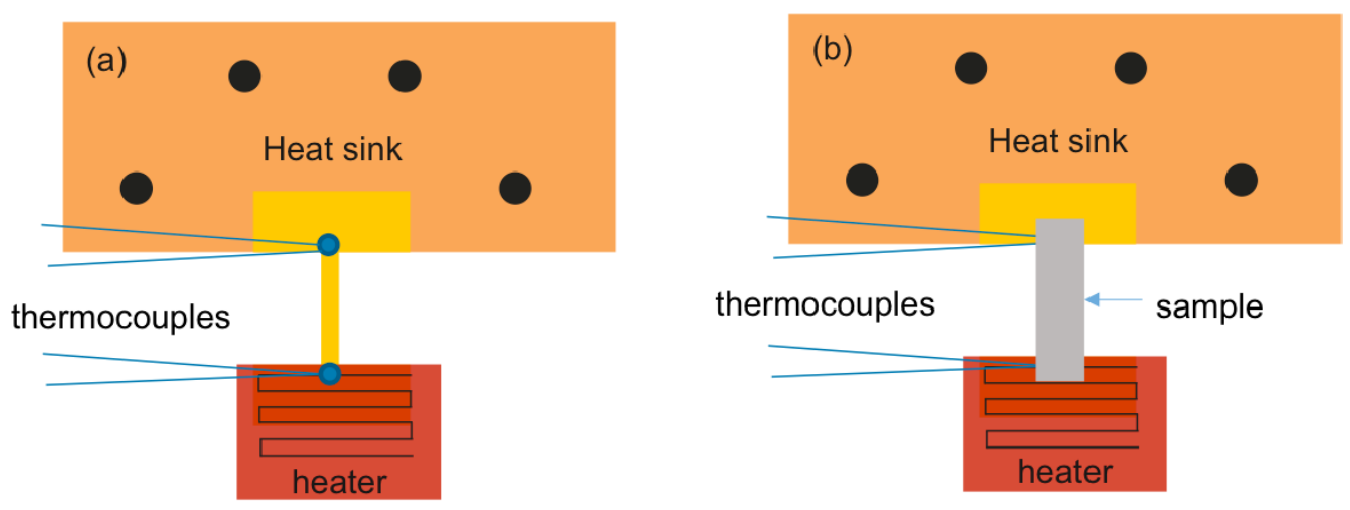

Figure 2.6 (a) sample holder for measuring in-plane thermal conductivity, (b) A thermoelectric film sample mounted in the sample holder.

As shown in Figure 2.6, a sample holder was fabricated to secure the film sample during measurement. A Kapton strip was used as the sample holder due to its low thermal conductivity, which minimizes the baseline thermal conductance. A strain gauge heater was attached to the hot side of the sample holder to provide heat sources while the cold side was mounted to a copper heat sink. The measurement was conducted in a vacuum cryostat chamber at $10^{-5}$ Torr to minimize convection losses. In addition, a radiation shield was surrounding the test setup to minimize radiation losses.

The first baseline measurement determines the baseline thermal conductance $\mathrm{C}_{\text {baseline }}$ of the sample holder using equation (1)

$$
\mathrm{P}=\mathrm{I}^{2} \mathrm{R}=\mathrm{C}_{\text {baseline }} \Delta \mathrm{T}
$$

The second measurement determines the total thermal conductance $\mathrm{C}_{\text {total }}$ of the sample and the sample holder.

$$
\mathrm{P}=\mathrm{I}^{2} \mathrm{R}=\mathrm{C}_{\text {total }} \Delta \mathrm{T}
$$


The thermal conductivity of the sample is determined using the following equation

$$
\mathrm{K}=\left(\mathrm{C}_{\text {total }}-\mathrm{C}_{\text {baseline }}\right) \cdot \mathrm{L} / \mathrm{A}
$$

To calibrate the test setup, the thermal conductivity of a $0.2 \mathrm{~mm}$ thick glass slide was measured, which was within $5 \%$ of the reference value.

The room-temperature lattice thermal conductivity $\kappa_{\mathrm{L}}$ of the film and the pellet is estimated to be $0.41 \mathrm{~W} / \mathrm{mK}$ and $0.66 \mathrm{~W} / \mathrm{mK}$ respectively using the equation $\kappa_{\mathrm{L}}=\kappa-\sigma \mathrm{TL}$, where $\kappa$ is the total thermal conductivity, $\sigma$ is the electrical conductivity, $\mathrm{T}$ is the absolute temperature, and $\mathrm{L}$ is the Lorenz number determined from our previous work ${ }^{22}$. The $\kappa_{\mathrm{L}}$ of these samples is significantly lower than their bulk counterpart attributed to the nanoscale grains and porosities originated from the nanocrystals ${ }^{22}$. Furthermore, the $\kappa_{\mathrm{L}}$ of the film is even lower than the pellet mainly due to additional phonon defects scattering caused by the addition of glass particles as well as a small contribution from slightly higher porosities. As shown in Figure 2.3(d), the film demonstrates a peak ZT of 0.43 at $175{ }^{\circ} \mathrm{C}$, which is only $20 \%$ lower than the control pellet despite $56 \%$ lower electrical conductivity. The significantly reduced thermal conductivity largely compensates the electrical conductivity losses and contributes to the high ZT in the printed films. In comparison, Table 2.1 shows the peak ZT (or room-temperature ZT if peak ZT is not available) of several representative n-type flexible thermoelectric materials, summarizing the highest reported ZT thus far in each category. The peak ZT of our flexible film is significantly higher than the previously reported bismuth telluride materials fabricated by printing, and is also among the highest reported value in all the reported n-type flexible thermoelectric films. In addition to the high ZT, our printed films 
are very stable in air and maintain the same thermoelectric properties in a wide range of thicknesses from tens to hundreds of microns that are very suitable for thermoelectric device fabrication

Table 2. 2 The thermoelectric performance comparison between our work and previous reported n-type flexible thermoelectric films.

\begin{tabular}{|c|c|c|c|c|}
\hline $\begin{array}{c}\text { Materials } \\
\text { details }\end{array}$ & $\begin{array}{c}\text { Power factor } \\
\left(\mathrm{mW} / \mathrm{mK}^{2}\right)\end{array}$ & $\begin{array}{c}\text { Peak/room } \\
\mathrm{T}^{*} \mathrm{ZT}\end{array}$ & Ref. & Fabrication methods \\
\hline $\mathrm{Bi}_{2} \mathrm{Te}_{2.8} \mathrm{Se}_{0.2}$ & 0.56 & 0.43 & $($ Ours $)$ & Screen printing \\
\hline $\mathrm{Bi}_{2} \mathrm{Te}_{3}$ & 1.33 & $0.35^{*}$ & 16 & Screen printing \\
\hline $\mathrm{Bi}_{2} \mathrm{Te}_{3}+$ Epoxy & 0.28 & $0.31^{*}$ & 15 & Dispenser printing \\
\hline $\mathrm{CNT}$ & 0.15 & N.A. & 23 & Drop casting \\
\hline $\mathrm{WS}_{2}$ & 0.007 & N.A. & 24 & Vacuum filtration \\
\hline $\mathrm{TiS}_{2}$-Polymer & 0.45 & 0.28 & 25 & Electrochemical intercalation \\
\hline CNT-PEDOT- & 1.05 & $\sim 0.5^{*}$ & 26 & Spraying and spin coating \\
TDAE & & & & \\
\hline
\end{tabular}

In order to test the flexibility, the room-temperature electrical resistivity of the printed films was tested using the Van der Pauw method vs. bending cycles on a cylinder of $7 \mathrm{~mm}$ radius. Electrical resistivity is chosen here to evaluate film flexibility because it is very sensitive to any cracks that may develop during bending test. As shown in Figure 2.7, the electrical resistivity shows only $1.4 \%$ increases after 150 bending cycles, indicating superior flexibility that are highly desired for many applications. Compared with $7 \mathrm{~mm}$ bending test, the resistance of the flexible film increased significantly, which exceeds $4 \%$ after 25 times bending; while for the $7 \mathrm{~mm}$ bending radius this value is only 
$1.4 \%$ even after 150 times bending. This indicates the super flexibility of the film under $7 \mathrm{~mm}$ bending radius. Also the bending test result is pretty sensitive to the bending radius; the smaller bending radius, the easier it to break due to the intrinsic brittle nature of inorganic thermoelectric powder

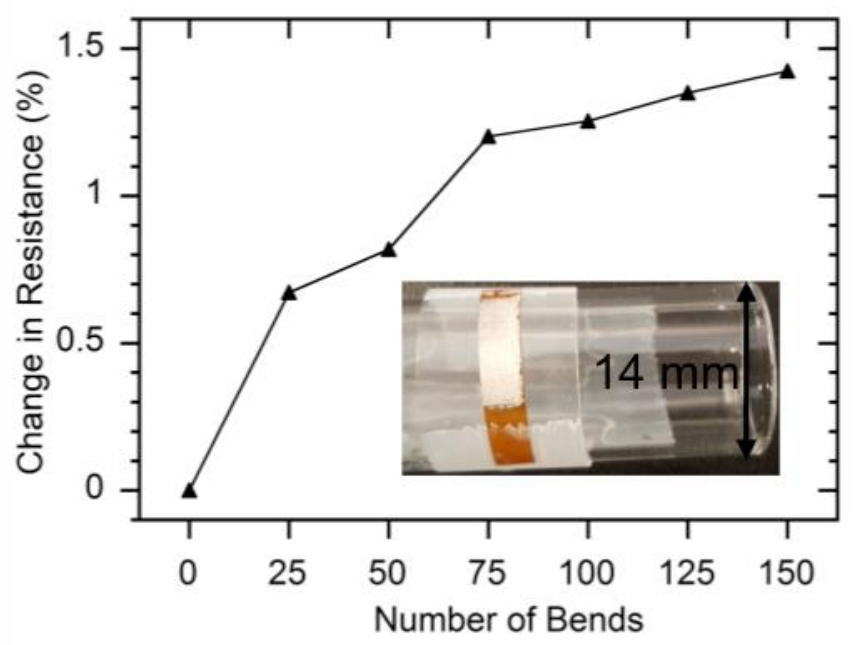

Figure 2. 7 Percentage increases of electrical resistivity of the flexible film vs. number of bending cycles. The inset is a flexible film bended on a glass tube of $14 \mathbf{~ m m}$ diameter.

\subsection{Conclusion}

In summary, flexible thermoelectric films were screen printed at ambient conditions using nanocrystals synthesized by a highly scalable microwave assisted wet chemical method. The films show an unprecedented peak ZT of 0.43 at $175^{\circ} \mathrm{C}$, and superior flexibility with negligible changes of electrical conductivity after 150 bending cycles. Using screen printing, thermoelectric nanocrystals can be directly converted into flexible thermoelectric devices through a very scalable and cost-effective process. The thermoelectric figure of merit of the printed films can be further increased by improving the electrical conductivity through improving the ink formulations and refining the sintering process. 


\subsection{Acknowledgements}

This work is funded by the US Department of Energy, Office of Nuclear Energy, under Award number DE-NE0008255. The authors thank Luke Schoensee for graphical support on this work.

\subsection{References}

1 Rowe, D. M. Thermoelectrics handbook: macro to nano. (CRC/Taylor \& Francis, 2006).

2 Bahk, J.-H., Fang, H., Yazawa, K. \& Shakouri, A. Flexible thermoelectric materials and device optimization for wearable energy harvesting. Journal of Materials Chemistry C, doi:10.1039/C5TC01644D (2015).

3 Goldsmid, H. J. Thermoelectric refrigeration. (Plenum Press, 1964).

4 Rowe, D. M. CRC handbook of thermoelectrics. (CRC Press, 1995).

5 Dresselhaus, M. S. et al. New directions for low-dimensional thermoelectric materials. Adv Mater 19, 1043-1053, doi:10.1002/adma.200600527 (2007).

6 Hicks, L. D. \& Dresselhaus, M. S. Effect of Quantum-Well Structures on the Thermoelectric Figure of Merit. Phys Rev B 47, 12727-12731, doi:DOI 10.1103/PhysRevB.47.12727 (1993).

7 Snyder, G. J. \& Toberer, E. S. Complex thermoelectric materials. Nat Mater 7, 105-114, doi:10.1038/nmat2090 (2008).

8 Minnich, A. J., Dresselhaus, M. S., Ren, Z. F. \& Chen, G. Bulk nanostructured thermoelectric materials: current research and future prospects. Energ Environ Sci 2, 466-479, doi:Doi 10.1039/B822664b (2009).

9 Shakouri, A. Recent Developments in Semiconductor Thermoelectric Physics and Materials. Annual Review of Materials Research, Vol 41 41, 399-431, doi:DOI 10.1146/annurev-matsci-062910-100445 (2011). 
10 Zhao, L. D., Dravid, V. P. \& Kanatzidis, M. G. The panoscopic approach to high performance thermoelectrics. Energ Environ Sci 7, 251-268, doi:Doi 10.1039/C3ee43099e (2014).

11 Yee, S. K., LeBlanc, S., Goodson, K. E. \& Dames, C. \$ per W metrics for thermoelectric power generation: beyond ZT. Energ Environ Sci 6, 2561-2571, doi:Doi 10.1039/C3ee41504j (2013).

12 Jiao, F. et al. Inkjet-printed flexible organic thin-film thermoelectric devices based on p- and n-type poly(metal 1,1,2,2-ethenetetrathiolate)s/polymer composites through ball-milling. Vol. 372 (2014).

13 Koplow, M., Chen, A., Steingart, D., Wright, P. K. \& Evans, J. W. in Medical Devices and Biosensors, 2008. ISSS-MDBS 2008. 5th International Summer School and Symposium on. 322-325.

14 Lu, Z. Y. et al. Fabrication of Flexible Thermoelectric Thin Film Devices by Inkjet Printing. Small 10, 3551-3554, doi:10.1002/smll.201303126 (2014).

15 Madan, D. et al. Enhanced Performance of Dispenser Printed MA n-type Bi2Te3 Composite Thermoelectric Generators. Acs Appl Mater Inter 4, 6117-6124, doi:10.1021/am301759a (2012).

16 Kim, S. J., We, J. H. \& Cho, B. J. A wearable thermoelectric generator fabricated on a glass fabric. Energ Environ Sci 7, 1959-1965, doi:10.1039/c4ee00242c (2014).

17 Kim, S. J., We, J. H., Kim, J. S., Kim, G. S. \& Cho, B. J. Thermoelectric properties of P-type Sb2Te3 thick film processed by a screen-printing technique and a subsequent annealing process. J Alloy Compd 582, 177-180, doi:10.1016/j.jallcom.2013.07.195 (2014).

18 Lee, H. B. et al. Thin-Film Thermoelectric Module for Power Generator Applications Using a Screen-Printing Method. J Electron Mater 40, 615-619, doi:10.1007/s11664-010-1481-0 (2011). 
19 We, J. H., Kim, S. J., Kim, G. S. \& Cho, B. J. Improvement of thermoelectric properties of screen-printed Bi2Te3 thick film by optimization of the annealing process. J Alloy Compd 552, 107-110, doi:10.1016/j.jallcom.2012.10.085 (2013).

20 Kamyshny, A. \& Magdassi, S. Conductive Nanomaterials for Printed Electronics. Small 10, 3515-3535, doi:10.1002/smll.201303000 (2014).

21 Mehta, R. J. et al. A new class of doped nanobulk high-figure-of-merit thermoelectrics by scalable bottom-up assembly. Nat Mater 11, 233-240, doi:http://www.nature.com/nmat/journal/v11/n3/abs/nmat3213.html\#supplementa ry-information (2012).

22 Zhang, Y. et al. Lattice thermal conductivity diminution and high thermoelectric power factor retention in nanoporous macroassemblies of sulfur-doped bismuth telluride nanocrystals. Appl. Phys. Lett. 100, 193113, doi:doi:http://dx.doi.org/10.1063/1.4711774 (2012).

23 Yu, C. H., Murali, A., Choi, K. W. \& Ryu, Y. Air-stable fabric thermoelectric modules made of N- and P-type carbon nanotubes. Energ Environ Sci 5, 94819486, doi:10.1039/c2ee22838f (2012).

24 Oh, J. Y. et al. Chemically exfoliated transition metal dichalcogenide nanosheetbased wearable thermoelectric generators. Energy Environ. Sci., doi:10.1039/C5EE03813H (2016).

25 Wan, C. L. et al. Flexible n-type thermoelectric materials by organic intercalation of layered transition metal dichalcogenide TiS2. Nat Mater 14, 622-627, doi:Doi 10.1038/Nmat4251 (2015).

26 Wang, H. et al. Thermally Driven Large N-Type Voltage Responses from Hybrids of Carbon Nanotubes and Poly(3,4-ethylenedioxythiophene) with Tetrakis(dimethylamino) ethylene. Adv Mater 27,6855, doi:10.1002/adma.201502950 (2015). 
CHAPTER THREE: BULK LIKE THERMOELECTRIC PERFORMANCES OF

FLEXIBLE DEVICES BY SCALABLE PRINTING AND LIQUID-PHASE SINTERING

*This chapter includes modifications from the originally submitted version. 
BULK LIKE THERMOELECTRIC PERFORMANCES OF FLEXIBLE DEVICES BY SCALABLE PRINTING AND LIQUID-PHASE SINTERING

\author{
Tony Varghese ${ }^{1}$ \\ Chaochao Dun ${ }^{2}$ \\ Nicholas Kempf ${ }^{2}$ \\ Mortaza Saeidi-Javash ${ }^{2}$ \\ Joseph Richardson ${ }^{3}$ \\ Courtney Hollar ${ }^{1}$ \\ David Estrada $^{1}$ \\ Yanliang Zhang ${ }^{2}$
}

${ }^{1}$ Micron School of Materials Science and Engineering, Boise State University, Boise, ID 83725, USA

${ }^{2}$ Department of Aerospace and Mechanical Engineering, University of Notre Dame, Notre Dame, IN 46556, USA

3 Department of Electrical and Computer Engineering, Boise State University, Boise, ID 83725, USA 


\subsection{Abstract}

Flexible thermoelectric devices are attractive power sources for the growing demand of flexible electronics and sensors. While many rigid bulk thermoelectric materials have shown a thermoelectric figure of merit ZT greater than unity, it has been an exacting challenge to develop flexible thermoelectric materials with ZT even near unity. Here we report a scalable and cost-effective screen-printing process combined with liquid-phase sintering to fabricate high-performance and flexible BiSbTe thermoelectric devices. The printed flexible films demonstrate a room-temperature power factor of 3 $\mathrm{mW} / \mathrm{m} \cdot \mathrm{K}^{2}$ and ZT about 1 , significantly higher than the previous reported values for flexible films. A fully printed flexible thermoelectric generator exhibits a high power density of $18.8 \mathrm{~mW} / \mathrm{cm}^{2}$ achievable with a reasonably small temperature gradient of 80 ${ }^{\circ} \mathrm{C}$. This screen-printing method, which directly transforms thermoelectric nanoparticles into high-performance and flexible devices, presents a significant leap to make thermoelectrics a commercially viable technology for a broad range of energy harvesting and cooling applications.

Keywords: Thermoelectric Generator; Flexible Film; Scalable Printing; LiquidPhase Sintering 


\subsection{Introduction}

The thermoelectric (TE) field has seen significant progress in increasing the figure of merit (ZT) in the past two decades. Their solid-state and compact nature makes thermoelectric generators (TEGs) produced from such high ZT materials a promising technology for capturing waste heat and boosting energy efficiency. Despite considerable ZT increases in the past two decades realized via nanostructuring and other techniques ${ }^{1-7}$ a huge chasm still remains to make TEGs a cost-competitive and commercially viable technology for a broad range of applications. The conventional TEG manufacturing process is expensive and inflexible, which is not adaptable for different applications. Advances in manufacturing methods are thus a paramount need to transform highefficiency nanostructured materials into cost-effective devices in order to realize the highly anticipated impact of thermoelectric technology. Printing techniques, such as direct writing, ${ }^{8-10}$ inkjet printing, ${ }^{11,12}$ and screen printing, ${ }^{13-16}$ have shown promise as scalable and cost-effective fabrication processes to realize flexible electronic and thermoelectric devices ${ }^{17,18}$. Among them, screen printing is a highly scalable, efficient, and low-cost technique to manufacture flexible thermoelectric devices.

Flexible thermoelectric generators (f-TEGs) have attracted great attention in recent years because of their broad range of applications as power sources for flexible electronics and wearable devices, which have rapidly growing markets. ${ }^{11,19-24}$ Research progresses on flexible TE materials and devices, including conducting polymers, ${ }^{25}$ nanocomposites comprised of inorganic nanostructures in polymer matrices ${ }^{26-28}$ and fully inorganic flexible thin films ${ }^{20,24}$ have been reported previously. ${ }^{29}$ The exponential growth in the Internet of Things (IoT) demands a large-scale, affordable, and uninterrupted 
power supply. Compared with other potential power sources, such as solar and piezoelectric devices, f-TEGs can continuously generate electricity from body/ambient thermal energy without the need for mechanical motion or sunlight. ${ }^{30,31}$ Nevertheless, the low thermoelectric power factor of printed flexible films compared with that of their rigid and bulk counterparts remains a major obstacle in applying f-TEGs in a broad range of energy harvesting and cooling applications. ${ }^{32}$ Moreover, there exist limited sintering options for the films printed on flexible substrates, leading to the formation of undesirable pores and thus reduced electrical conductivity ${ }^{12,33}$. Recently, attempts have been made to improve the electrical conductivity of TE materials fabricated from nanocrystals using nano-solder, ${ }^{34,35}$ but this method is not compatible with scalable printing at ambient conditions due to the complicated chemical synthesis involving hydrazine.

Here we report a highly scalable and low-cost screen-printing process to fabricate flexible and high-performance $p$-type films using ball-milled $\mathrm{Bi}_{0.4} \mathrm{Sb}_{1.6} \mathrm{Te}_{3}$ nanoparticles. A liquid-phase sintering process with additional tellurium particles was successfully employed to improve the density and charge carrier mobility of the printed films. The printed films demonstrate a thermoelectric power factor of $3 \mathrm{~mW} / \mathrm{m} \cdot \mathrm{K}^{2}$ and ZT about 1 at room temperature accompanied by superior flexibility, which is unprecedented for flexible films. A functional f-TEG was fabricated that exhibits a power density of 18.8 $\mathrm{mW} / \mathrm{cm}^{2}$ at a temperature difference of $80^{\circ} \mathrm{C}$. 


\subsection{Results and Discussion}

High-energy ball-milled $\mathrm{Bi}_{0.4} \mathrm{Sb}_{1.6} \mathrm{Te}_{3}$ (BST) nanoparticles ${ }^{36}$ were used to synthesize printable thermoelectric ink. The fabrication and liquid-phase sintering process for a f-TEG using scalable and low-cost screen printing is shown in Figure 3.1

Ball milled thermoelectric nanoparticles are mixed with binders and solvents to make thixotropic paste and transformed in to a flexible thermoelectric generator using screen printing. The printed and sintered samples were further analyzed XRD as shown in Figure 3.2, showing the presence of excess tellurium in the BST: T samples forming a separate region in the films.
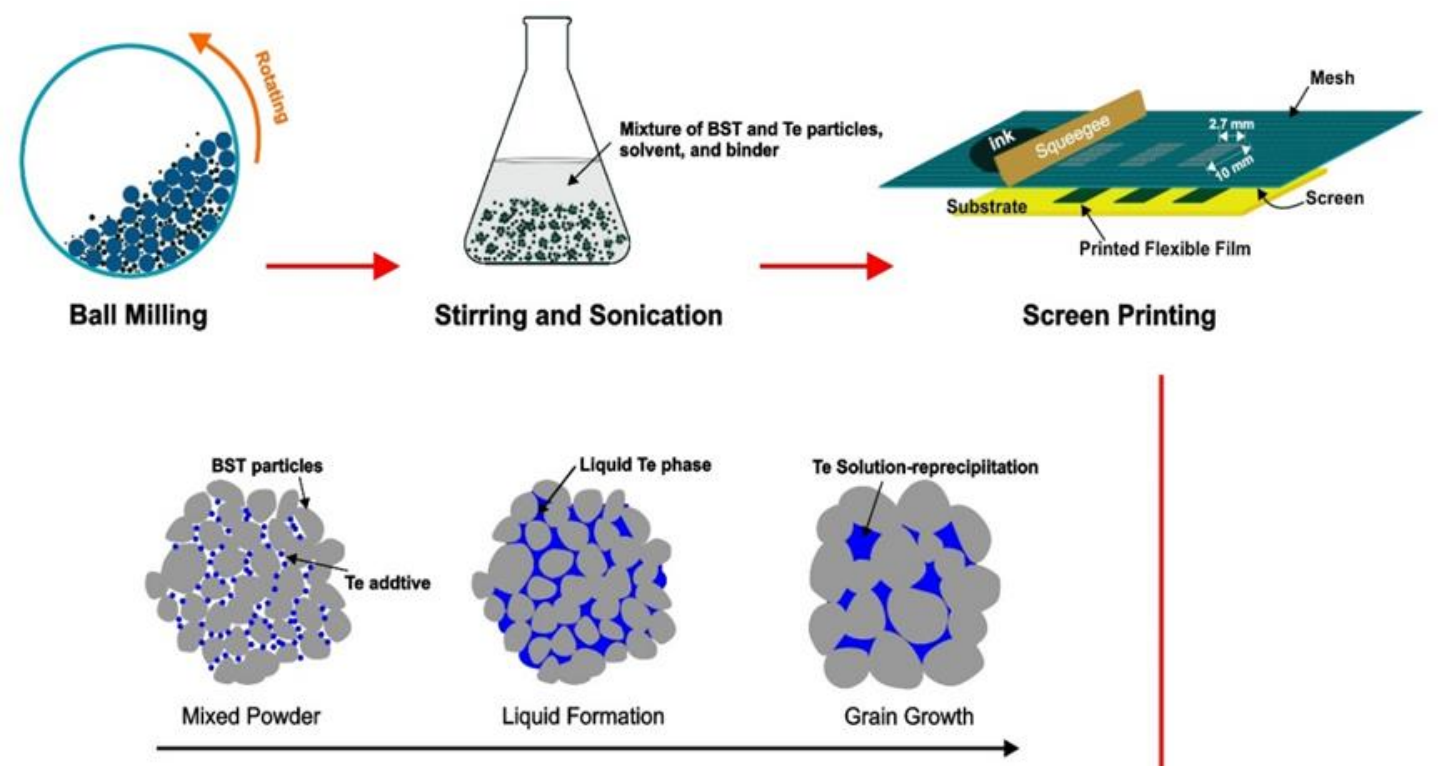

Liquid-phase Sintering

Figure 3.1 Converting Bio.4Sb1.6Te3 (BST) nanoparticle ink into a flexible thermoelectric generator using a scalable and low-cost screen-printing and liquidphase sintering process. 


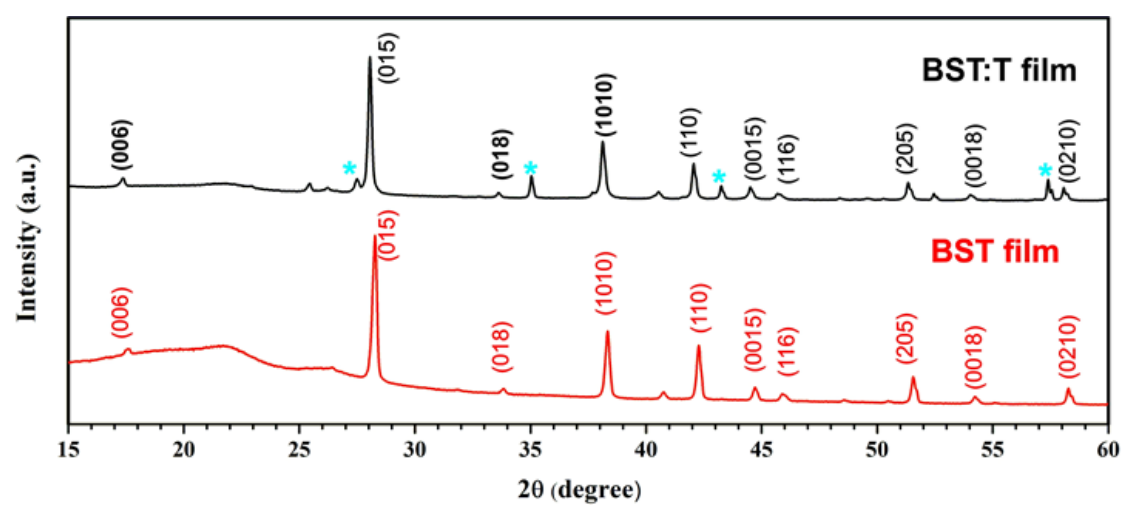

Figure 3. 2 X-ray diffraction pattern comparison between BST and BST:T samples, which verifies the presence of excess elemental tellurium (marked as blue stars) in the BST:T samples that is in agreement with the EDS.

3.3(a-b) shows the comparison between a scanning electron microscope (SEM) image of the printed BST film with $8 \mathrm{wt} . \%$ addition of tellurium particles (BST:T) and that of a pure BST film after sintering at $450{ }^{\circ} \mathrm{C}$. It is clear that the BST:T sample has denser structures with larger grain size and fewer pores.3.3(a) and (c) shows the tellurium-rich areas in the BST:T film, as compared with the uniform distribution of Bi, $\mathrm{Sb}$, and Te in the pure BST film shown in 3.3(b) and (d). Elemental ratio analysis of the main phase of each film reveals the Bi:Sb:Te element ratio to be $0.4: 1.6: 3$, while the areas marked with dashed circles in Figure 3.3(a) are dominated by the re-precipitation of tellurium with Te element ratio exceeding $88 \%$. Details of the EDS analysis are provided in Figure 3.4. 

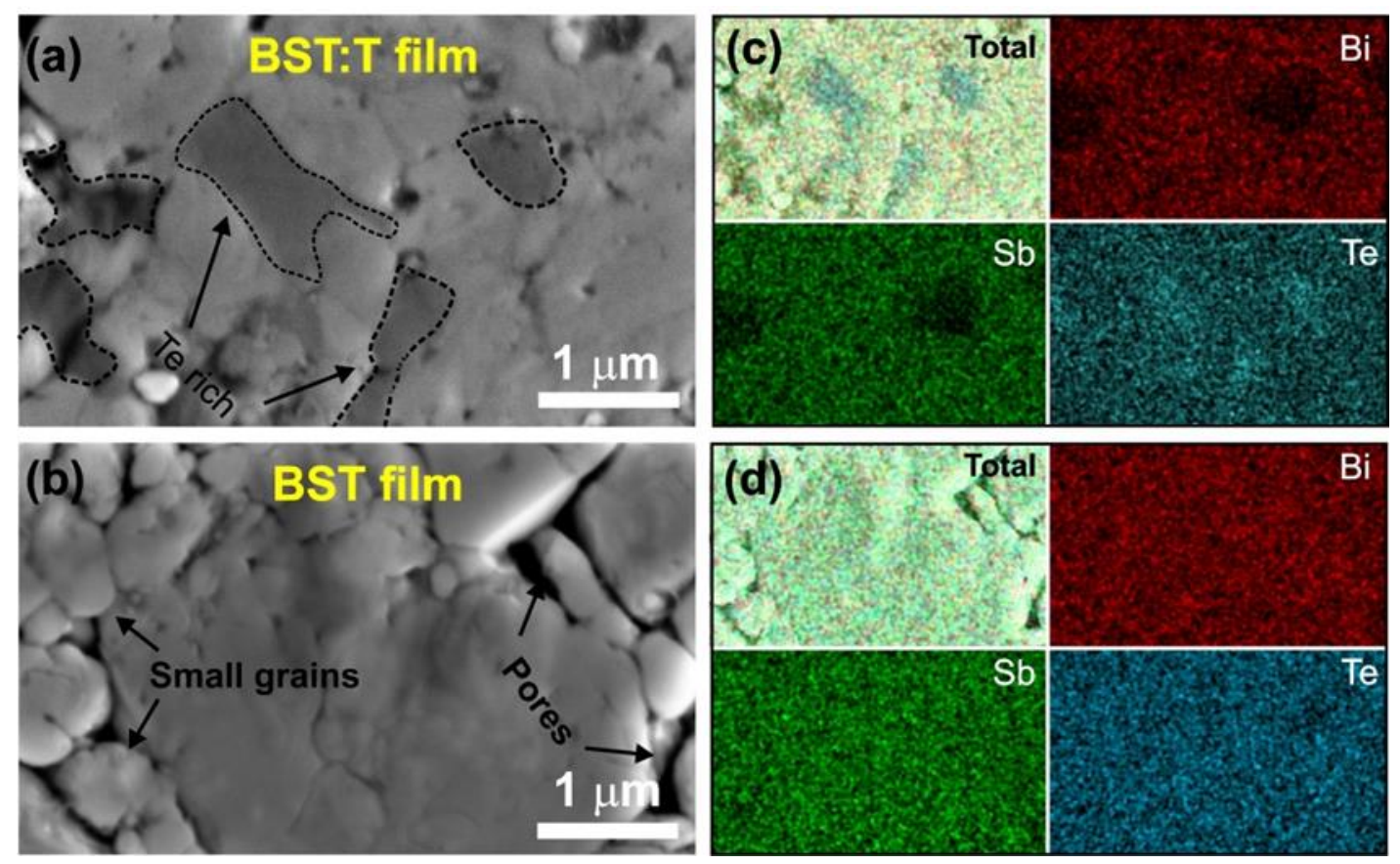

Figure 3. 3 SEM images of (a) printed Bio.4Sb1.6Te3 films with 8 wt. \% Te and (b) printed pure Bio.4Sb1.6Te3 films. The corresponding energy-dispersive X-ray spectroscopy (EDS) mapping is given in (c) and (d), respectively

During the sintering process at $450{ }^{\circ} \mathrm{C}$, the excess tellurium particles, with melting temperature $<449^{\circ} \mathrm{C}$, creates a liquefied tellurium phase in the solid BST matrix. The introduced liquid-tellurium phase acts as a coalescing agent to facilitate the consolidation and grain growth of the BST nanoparticles, contributing to enhanced film density. Temperature-dependent thermoelectric properties were systematically studied based on printed films with an average thickness around $27 \mu \mathrm{m}$. As shown in Figure3.6 (a), both BST and BST:T systems show decreased electrical conductivity with increased temperature, which is consistent with their semi-metallic nature. The room-temperature electrical conductivity of the BST:T film is $42 \%$ higher than that of the BST film. It is 

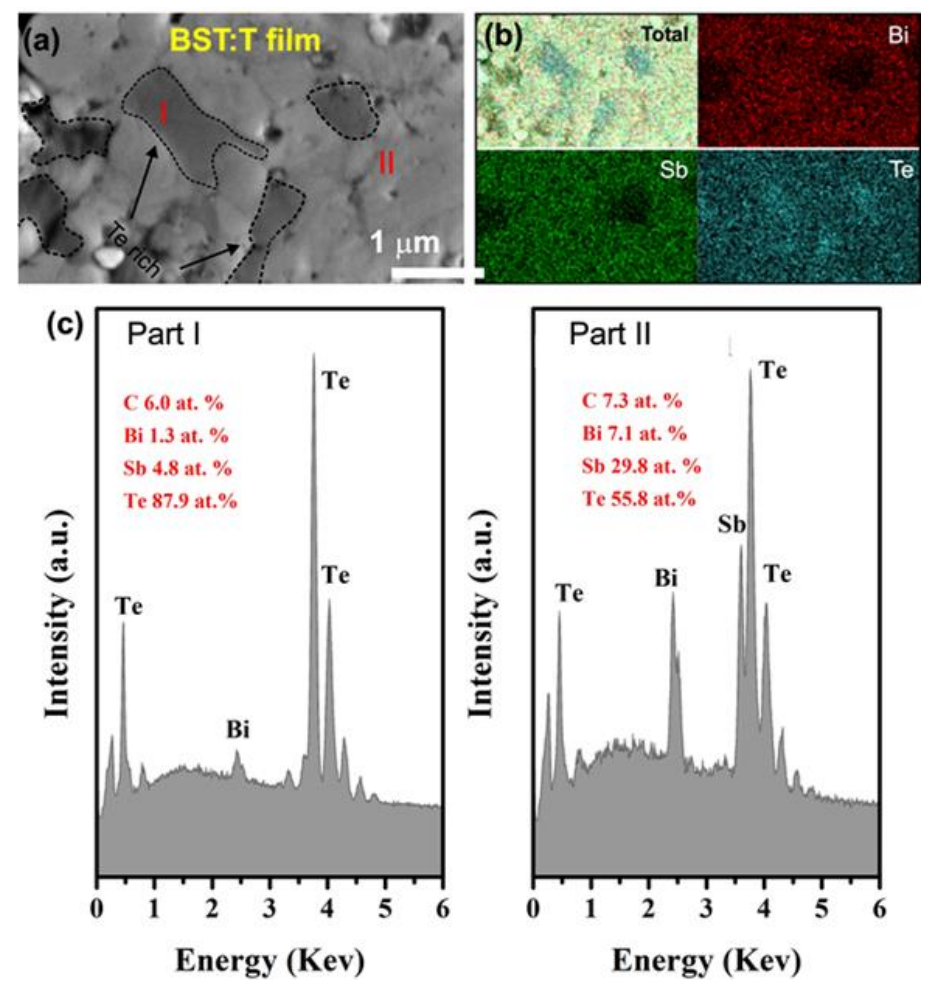

Figure 3. 4 (a) SEM of BST:T film with (b) the corresponding EDS analysis, (c) detailed elements ratios for the part I and II as marked in (a). It is observed that the re-precipitation of Te occurs, which increase the film density that is helpful for the increased electrical conductivity. The main body (part II) has the same element ratios of Bi-Sb-Te as the original particles (Bio.4Sb1.6Te3).

believed that the tellurium addition results in: (I) diminished carrier scattering due to the increase in grain size and film density that occurs during the liquid-phase sintering process; and (II) an increased carrier (hole) concentration, which is closely related to the modulation of anti-sites $\left(\mathrm{Sb}_{\mathrm{Te}}^{-}\right.$and $\left.\mathrm{Bi}^{-} \mathrm{Te}\right)$ and anion vacancy $\left(\mathrm{V}_{\mathrm{Te}^{2+}}\right)$ defects in the BST matrix. ${ }^{37,38}$ In order to confirm the change of carrier concentration and mobility, roomtemperature Hall measurements were performed on a series of flexible films. An increased carrier concentration from $2.4 \times 10^{19}$ to $3.1 \times 10^{19} \mathrm{~cm}^{-3}$, as well as an increased carrier mobility from 111.4 to $135.9 \mathrm{~cm}^{2} \mathrm{~V}^{-1} \mathrm{~s}^{-1}$, were observed in the BST:T film as 

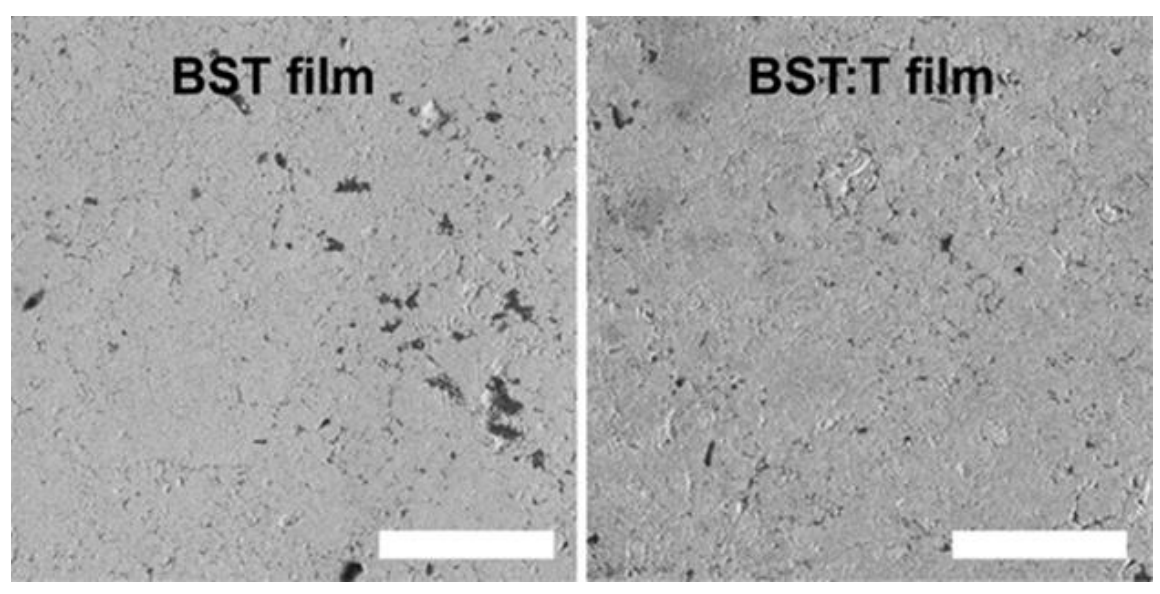

Figure 3.5 SEM images of the BST films without (a) and with (b) additional Te with lower magnifications. All scar bars are $25 \mu \mathrm{m}$. It is shown that the BST:T films are more dense with less porosity compared with that of BST films

compared with the BST film. Normally, the carrier mobility decreases with increased carrier concentration due to the increased carrier-carrier scattering. However, the introduction of additional Te in the BST:T system could be conducive to the synergistic effect of increased carrier concentration accompanied by the suppressed scattering of charge carriers as a result of the reduced porosity and increased grain size after liquidphase sintering. Specifically, it is believed that the excess Te particles creates a liquefied tellurium phase in the solid BST matrix, which acts as a coalescing agent to facilitate the consolidation of the BST nanoparticles. As a result, the melted tellurium at the BST interfaces can not only slightly increase carrier concentration, but also improve the networks between the BST particles and thus improve the carrier mobility by reducing the porosity of the films and carrier scattering at the pore boundaries. ${ }^{35}$ Therefore, the simultaneously improved carrier concentration and mobility finally gives rise to the significantly enhanced electrical conductivity in the printed BST:T film, comparable to 
that of a commercial BST bulk ingot.
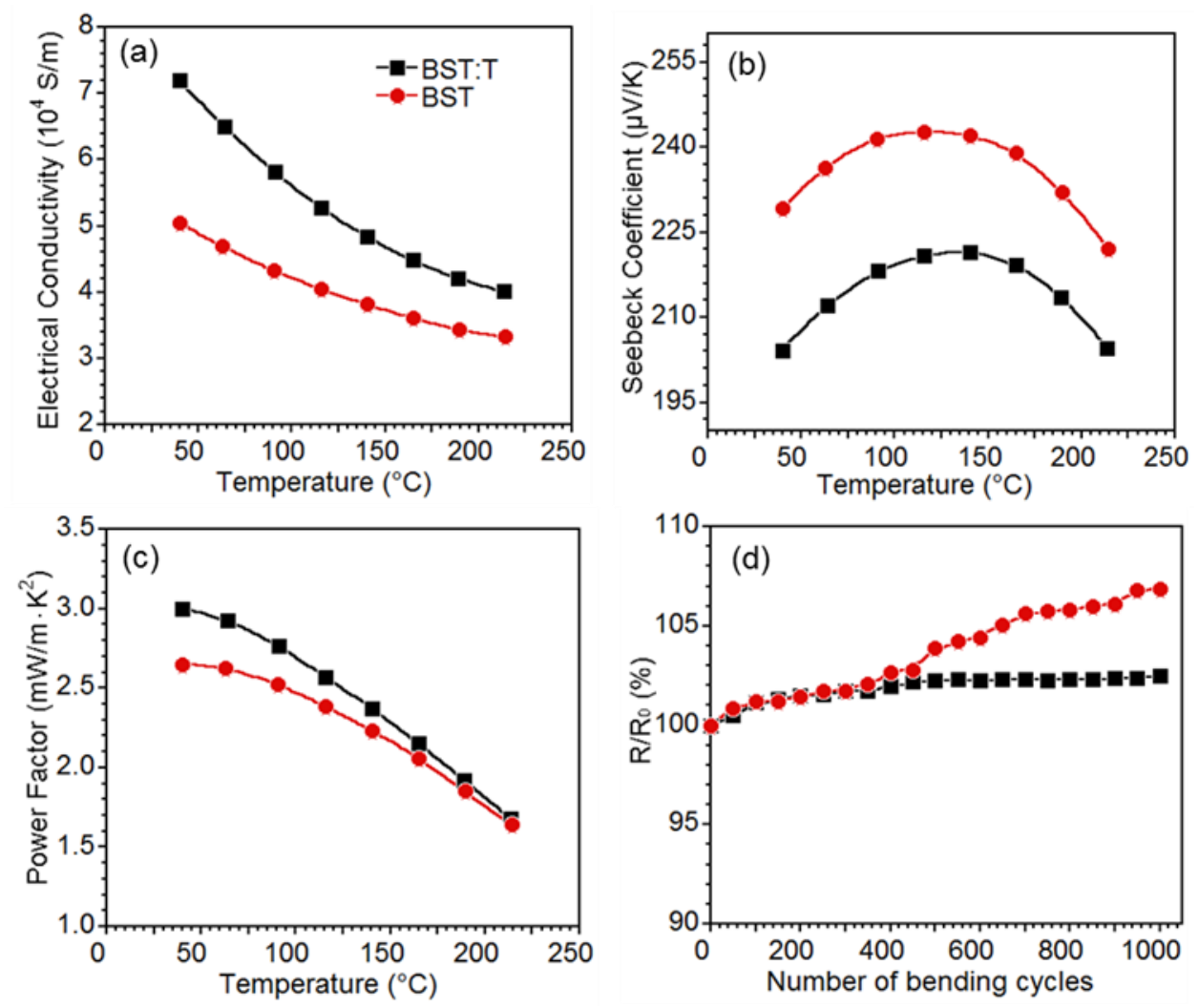

Figure 3. 6 Temperature-dependent (a) electrical conductivity, (b) Seebeck coefficient, and (c) power factor of both BST and BST:T films. (d) Normalized resistance of flexible films vs. number of bending cycles with a $10 \mathrm{~mm}$ bending radius for both BST and BST:T films. Here, $R_{0}$ and $R$ are the resistances before and after bending.

With the tellurium addition, the Seebeck coefficient of the BST:T films decreases slightly from 230 to $204 \mu \mathrm{V} / \mathrm{K}$, as shown in Figure 3.6(b). The reduced Seebeck coefficient is associated with the increased charge-carrier concentration as discussed above. At the same time, the Seebeck coefficient of BST:T films first increases and then starts to decrease at the higher temperature of $150^{\circ} \mathrm{C}$ as compared with $105^{\circ} \mathrm{C}$ for pure BST films. This is because the excess tellurium reduces the density of positively charged tellurium vacancies (minority carriers), ${ }^{35}$ decreasing the extent of the bipolar effect, 
which is in agreement with the Hall-measurement results. Figure 3.6(c) shows the roomtemperature power factor of the BST:T film is $13 \%$ higher than that of the pure BST film due to its significantly increased electrical conductivity. The room-temperature thermal conductivity of the BST:T film and the BST film were determined to be $0.9 \mathrm{Wm}^{-1} \mathrm{~K}^{-1}$ and $0.95 \mathrm{Wm}^{-1} \mathrm{~K}^{-1}$ using the using the Angstrom method, as shown in Figure 3.7. This results in a room-temperature ZT value about 1 for the BST:T films, which is significantly higher than the previous best reported ZT values of flexible films, as shown in Table 1. The thermal conductivity of the BST:T film is slightly lower than the BST film because the secondary tellurium phase presented in the BST:T film increases phonon scattering and thus reduces the lattice thermal conductivity. Table 1 summarizes the roomtemperature power factor and ZT values of several $p$-type flexible films fabricated by different methods, showing that BST:T has the highest reported values thus far in both categories. The room-temperature power factor of our printed BST:T films reaches 3 $\mathrm{mW} / \mathrm{m} \cdot \mathrm{K}^{2}$, which is at least $120 \%$ higher than the previous best reported values of $p$-type flexible thermoelectric films.

The TE films were printed on polyimide substrate with $51 \mu \mathrm{m}$ thickness. The thickness of the TE films in the range of 24-28 $\mu$ m measured using SEM. The thermal diffusivity measurements were performed on both pristine polyimide and the screen printed TE film on polyimide separately. Then, modified effective medium theory ${ }^{47}$ was 

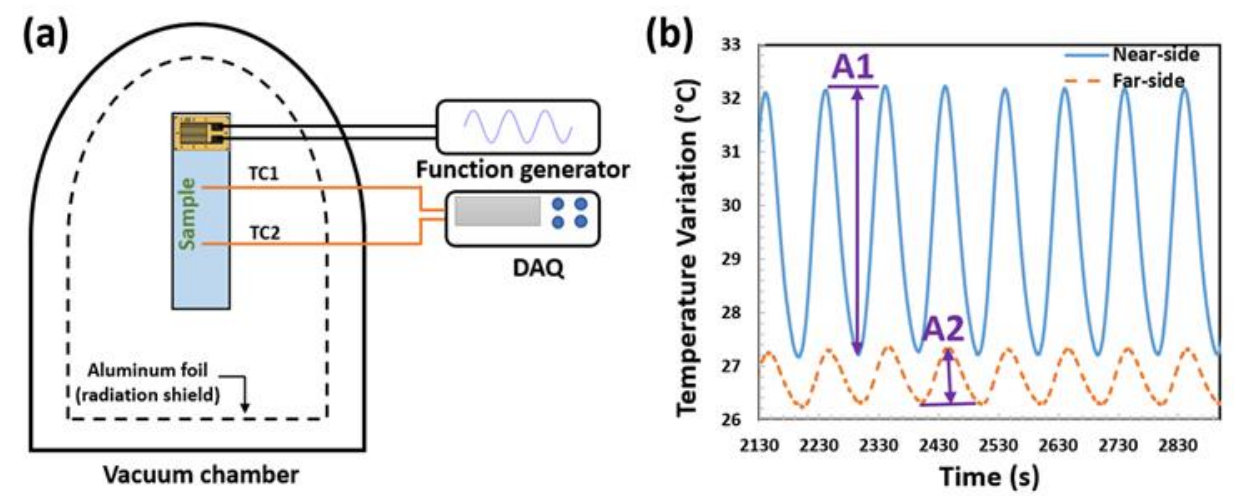

Figure 3. 7 (a) Schematic of the Angstrom measurement setup; (b) measured temperature waves of the two thermocouples on the sample

used to extract the thermal conductivity of the TE film itself, according to the following equation:

$$
\alpha_{\text {combined }}\left(\rho_{\text {film }} c_{\text {film }} \frac{A_{\text {film }}}{A_{\text {sub }}}+\rho_{\text {sub }} c_{\text {sub }}\right)=k_{\text {film }} \frac{A_{\text {film }}}{A_{\text {sub }}}+k_{\text {sub }}
$$

Where $\alpha_{\text {combined }}$ is the thermal diffusivity measured on the combined TE film and substrate, $\rho_{\text {film }}, c_{\text {film }}, A_{\text {film }}, k_{\text {film }}$ are the density, specific heat, cross-section area and thermal conductivity of the TE film respectively. Similarly, $\rho_{\text {sub }}, c_{\text {sub }}, A_{\text {sub }}$ and $k_{\text {sub }}$ are the density, specific heat, cross-section area and thermal conductivity of the substrate. To minimize the heat loss effect through radiation, the sample was surrounded with an aluminum foil radiation shield. Moreover, the accuracy of the measurement setup was validated by good agreement $(<3 \%)$ between the measured thermal diffusivity and the reported values in literature of samples with well-known thermal diffusivity (e.g. polyethylene, fused quarts).

In order to test the flexibility of the printed films, the room-temperature electrical resistance was tested using a Van der Pauw method as a function of bending cycles with a 
$10 \mathrm{~mm}$ bending radius. Electrical resistance was chosen to evaluate the film flexibility because it is highly sensitive to any cracks that may develop during bending test. As shown in Figure 3.6(d), the BST:T film and the BST film show 3\% and 7\% increases in resistance, respectively, after 1000 bending cycles, indicating the superior flexibility of the printed film with liquid-phase sintering. No noticeable changes of Seebeck coefficient were observed before and after bending.

Finally, a f-TEG consisting of four BST:T TE elements (shown in the inset of Figure 3.8(a)) was printed, and tested at various temperature differences $(\Delta T)$ with the hot side varying in the range of $40-100^{\circ} \mathrm{C}$ and the cold side maintained at $20^{\circ} \mathrm{C}$. Figure 3.8 (a) shows that the experimentally measured device open circuit voltage linearly increases with $\Delta \mathrm{T}$ by virtue of the Seebeck effect, which agrees with the calculated results within $5 \%$ at $80{ }^{\circ} \mathrm{C} \Delta \mathrm{T}$. Figure $3.8(\mathrm{~b}, \mathrm{~d})$ shows the device operating voltage and power output, respectively, as a function of electrical current tested at different $\Delta T$. A maximum power output of $54 \mu \mathrm{W}$ was obtained with a $\Delta \mathrm{T}$ of $80^{\circ} \mathrm{C}$ when the external load resistance matches the internal resistance of the device. The device power density increases with $\Delta \mathrm{T}$ and reaches $18.8 \mathrm{~mW} / \mathrm{cm}^{2}$ at $80^{\circ} \mathrm{C} \Delta \mathrm{T}$, as shown in Error! Reference s ource not found.9 (c). It should be noted that the power density is one of the most important criteria to evaluate the performance of TEG energy harvesters. Here, the power density was evaluated based on the total cross-sectional area of the four thermoelectric elements perpendicular to the heat flow direction. The differences between the experimental power density and the calculated values increases slightly with $\Delta \mathrm{T}$ due to the increasing electrical contact resistances at the junctions between the metal electrodes and the TE elements. The fully printed flexible thermoelectric generator with a power 
density of $18.8 \mathrm{~mW} / \mathrm{cm}^{2}$ at $80^{\circ} \mathrm{C} \Delta \mathrm{T}$ is sufficient to power the Internet of Things and sensors with typical power consumptions from $100 \mathrm{nW}$ to $100 \mathrm{~mW}{ }^{45}$.

Table 3.1 Comparison of room-temperature thermoelectric performance between this work and previous best reported values of p-type flexible thermoelectric films.

\begin{tabular}{|c|c|c|c|c|}
\hline Composition & $\begin{array}{l}\text { Power factor } \\
\left(\mathrm{mW} / \mathrm{m} \cdot \mathrm{K}^{2}\right)\end{array}$ & ZT & $\begin{array}{c}\text { Fabrication } \\
\text { method }\end{array}$ & Reference \\
\hline BiSbTe+ 8 wt. $\%$ Te & 3.0 & 1 & Screen-printing & This work \\
\hline BiSbTe & 0.06 & $\mathrm{n} / \mathrm{a}$ & Ink-Jet printing & 11 \\
\hline BiSbTe & 0.18 & 0.2 & Direct-write & 10 \\
\hline $\begin{array}{c}\text { p-type } \mathrm{Bi}_{2} \mathrm{Te}_{3} /(\mathrm{PEDOT}) \\
\text { hybrid }\end{array}$ & 1.35 & 0.58 & $\begin{array}{c}\text { Thermal } \\
\text { Evaporation }\end{array}$ & 39 \\
\hline Doped CNT & 0.7 & 0.1 & Spray-coating & 40 \\
\hline Hybrid complex & 0.06 & 0.3 & Drop-casting & 41 \\
\hline $\begin{array}{l}\text { Doped poly( } 3,4- \\
\text { ethylenedioxythiophene) }\end{array}$ & 0.45 & 0.42 & Spin-casting & 42 \\
\hline $\mathrm{CuI}$ & 0.4 & 0.21 & Sputtering & 43 \\
\hline $\mathrm{Ca}_{3} \mathrm{Co}_{4} \mathrm{O}_{9}$ & 0.23 & $\mathrm{n} / \mathrm{a}$ & Sputtering & 44 \\
\hline
\end{tabular}



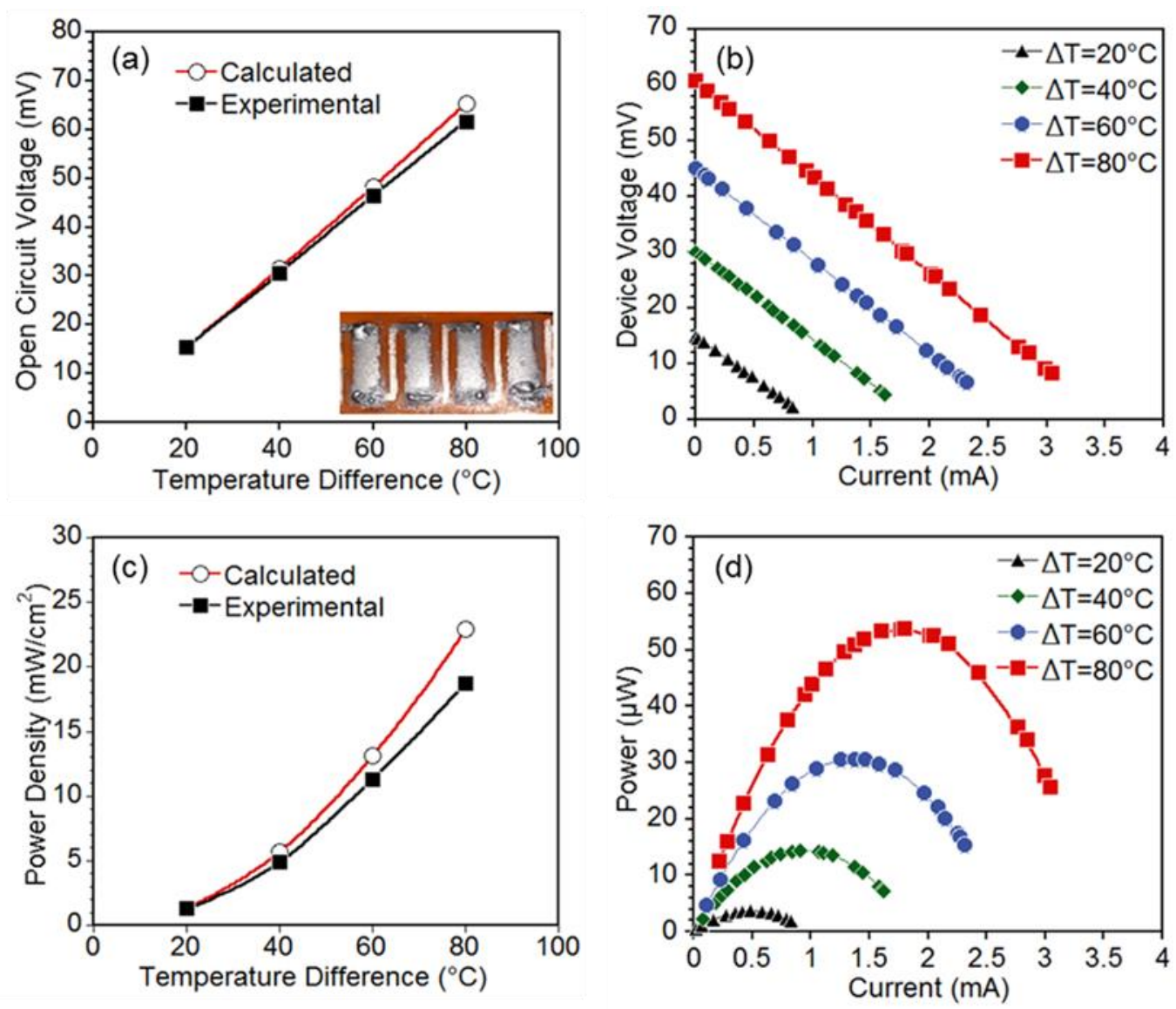

Figure 3.8 Performance of a flexible TE device fabricated by the screen-printing and liquid-phase sintering process: (a) calculated and experimental open circuit voltage vs. temperature differences $(\Delta T)$ (Inset: photo of the printed device); (b) device operating voltage vs. current tested at various $\Delta \mathrm{T}$; (c) calculated and experimental electrical power density tested at various $\Delta T$; and (d) power output vs. electrical current at various $\Delta \mathrm{T}$.

The screen-printed thermoelectric devices based on flexible films have potential to significantly reduce thermoelectric materials cost compared with bulk devices with TE element thickness of millimeters due to reduced materials consumptions. In addition, the direct conversion of thermoelectric particles into devices realized by the screen-printing process not only eliminates materials waste but also reduces manufacturing cost compared with conventional device manufacturing method which typically involves 
numerous processes such as powder hot press, polishing, dicing, and assembling and joining TE elements with metal electrodes. ${ }^{46}$

\subsection{Conclusion}

In conclusion, flexible BiSbTe based thermoelectric devices were fabricated using a highly scalable and low-cost screen-printing method. A bulk-like power factor of 3 $\mathrm{mW} / \mathrm{m} \cdot \mathrm{K}^{2}$ and a ZT about 1 were achieved at room temperature, which is significantly higher than previous reported values of flexible TE films and is ideal for near roomtemperature energy harvesting applications. Bending test revealed excellent flexibility of the printed films, which exhibit only a 3\% increase in electrical resistance after 1000 bending cycles on a $10 \mathrm{~mm}$ radius cylinder. The flexible thermoelectric device fabricated using printed BiSbTe films and silver electrodes exhibits an exceptional power density of $18.8 \mathrm{~mW} / \mathrm{cm}^{2}$ at a relatively small temperature difference of $80^{\circ} \mathrm{C}$. The flexible, highperformance, and low-cost thermoelectric devices demonstrated in this work present a significant leap in the field of thermoelectrics toward a commercially viable technology for a broad range of energy-harvesting and thermal-management applications.

\subsection{Experimental Procedures}

It was determined that a small amount of tellurium particles can be mixed with the $\mathrm{Bi}_{0.4} \mathrm{Sb}_{1.6} \mathrm{Te}_{3}$ particles to introduce liquid-phase sintering and help consolidate the $\mathrm{Bi}_{0.4} \mathrm{Sb}_{1.6} \mathrm{Te}_{3}$ particles. The tellurium particles melt during sintering when the temperature is above its melting point but below the BST melting point. A suitable amount of TE powder was weighed and mixed with a measured amount of solvent and binder. Here, the optimized ink contains 84 wt. \% TE particles, 13 wt. \% solvent ( $\alpha$-Terpineol, SigmaAldrich), and 3 wt. \% binder (Disperbyk-110, BYK U.S.A. Inc.). The ink was well mixed 
using a planetary centrifugal mixer for 20 minutes followed by a vortex mixer for 10 minutes to obtain uniformity.

The TE ink was printed onto a $51 \mu \mathrm{m}$ thick HN-Kapton substrate using 180 mesh screens. Before printing, Kapton films were cut to required dimensions and cleaned using methanol and isopropanol by sonication. Multiple passes of printing were applied to obtain the desired film thickness and uniformity. Freshly printed films were allowed to rest for 30 minutes in their initial state, allowing reduction of the mesh pattern on the films. The printed films were then dried at $200^{\circ} \mathrm{C}$ for 30 minutes in a nitrogen environment in order to remove the solvent and binder. The dried samples were further densified using a hydraulic press with pressure up to $200 \mathrm{MPa}$. Finally, the pressed films were sintered at $450^{\circ} \mathrm{C}$ for 45 minutes using a tube furnace under inert environment. The sintering temperature was kept below the melting point of the Kapton substrate.

In order to fabricate a thermoelectric device, a series of sintered films were electrically connected using screen-printed silver contacts (HPS-FG57B silver ink, Novacentrix) and flash-dried at $350^{\circ} \mathrm{C}$ for one minute. A completely printed device was fabricated using 4 thermoelectric elements with $27 \mu \mathrm{m}$ thickness connected by silver prints. The total weight of the printed thermoelectric generator with electrical contacts is under $19 \mathrm{mg}$. The thickness, microstructure, and elemental compositions of the films were characterized using field emission-scanning electronic microscopy (FE-SEM, Teneo) equipped with energy dispersive spectroscopy (EDS).

The temperature-dependent in-plane electrical conductivity and Seebeck coefficient of the film were measured using a commercial Linseis Seebeck and resistivity instrument (LSR system, Germany). The thermoelectric properties were measured at least 
twice on each film, and both Seebeck coefficient and electrical conductivity are within $2 \%$ between multiple measurements. The Hall measurement was conducted on a modified Hall Effect measurement system (Ecopia HMS5000/AMP55T) at a permanent magnetic field of $0.545 \mathrm{~T}$ in a Van der Pauw configuration. The room-temperature thermal conductivity $k$ of the printed films was determined through the measurement of the thermal diffusivity $\alpha$, specific heat capacity $c_{\mathrm{p}}$ and density $\rho$, using the relationship $k$ $=\alpha \rho c_{p}$. Thermal diffusivity was measured using the Angstrom method by applying a sinusoidal heat signal at one end of the sample and measuring the temperature response as a function of time at two different locations along the sample. The thermoelectric device was measured using a custom-built testing system with controlled hot-side and cold-side temperatures, and a variable resistor to match the TE device resistances in order to obtain the maximum power output.

The crystallization of all films was analyzed by X-ray diffraction (XRD) using $\mathrm{Cu}$ K $\alpha$ radiation $(\lambda=1.5418 \AA$, Bruker D2 Phaser). Scanning Electron Microscopy and related Energy-dispersive X-ray spectroscopy tests were performed using Magelllan 400 (FEI Company), with working voltage $15 \mathrm{KV}$ and working distance $4.5 \mathrm{~mm}$. Transmission Electron Microscope (TEM) and High Resolution TEM (HRETM) including the selected area electron diffraction (SAED) images were performed using a Titan 80-300 (FEI).

\subsection{Acknowledgments}

We acknowledge funding support from the National Science Foundation under award CMMI-1747685, and DARPA under DARPA HR00111820030. T.V. and D.E. acknowledge support from the National Aeronautics Space Administration under award 
80NSSC17M0029. The authors thank Dr. Zhifeng Ren and Dr. Dezhi Wang at University of Houston for providing the TE powders for this study.

\subsection{References}

1. Tan, G.; Zhao, L. D.; Kanatzidis, M. G. Rationally Designing High-Performance Bulk Thermoelectric Materials. (2016). Chem. Rev. 116 (19), 12123-12149.

2. Biswas, K.; He, J.; Blum, I. D.; Chun-IWu; Hogan, T. P.; Seidman, D. N.; Dravid, V. P.; Kanatzidis, M. G. (2012). High-performance bulk thermoelectrics with allscale hierarchical architectures. Nature. 489, 414-418.

3. Zhao, L.-D.; Lo, S.-H.; Zhang, Y.; Sun, H.; Tan, G.; Uher, C.; Wolverton, C.; Dravid, V. P.; Kanatzidis, M. G. (2014). Ultralow thermal conductivity and high thermoelectric figure of merit in SnSe crystals. Nature. 508, 373-777.

4. Minnich, A. J.; Dresselhaus, M. S.; Ren, Z. F.; Chen, G. (2009). Bulk nanostructured thermoelectric materials: current research and future prospects. Energy Environ Sci. 2, 466-479.

5. Mehta, R. J.; Zhang, Y.; Karthik, C.; Singh, B.; Siegel, R. W.; Borca-Tasciuc, T.; Ramanath, G. (2012). A new class of doped nanobulk high-figure-of-merit thermoelectrics by scalable bottom-up assembly. Nat Mater. 11 (3), 233-240.

6. Jood, P.; Ohta, M.; Yamamoto, A.; Kanatzidis, M. G. (2018). Excessively Doped PbTe with Ge-Induced Nanostructures Enables High-Efficiency Thermoelectric Modules. Joule. 2 (7), 1339-1355.

7. Sun, L.; Liao, B.; Sheberla, D.; Kraemer, D.; Zhou, J.; Stach, E. A.; Zakharov, D.; Stavila, V.; Talin, A. A.; Ge, Y.; Allendorf, M. D.; Chen, G.; Léonard, F.; Dincă, M. (2017). A Microporous and Naturally Nanostructured Thermoelectric MetalOrganic Framework with Ultralow Thermal Conductivity. Joule. 1, 168-177.

8. Gao, M.; Li, L.; Li, W.; Zhou, H.; Song, Y. (2016). Lead-Free Nanowire Aligned Flexible Piezoelectric Device. Adv. Sci. 3, 1600120.

9. Madan, D.; Chen, A.; Wright, P. K.; Evans, J. W.; Madan, D.; Chen, A.; Wright, P. K.; Evans, J. W. (2011). Dispenser printed composite thermoelectric thick 
films for thermoelectric generator applications Dispenser printed composite thermoelectric thick films for thermoelectric generator applications. J. Appl. Phys. 109, 034904.

10. Madan, D.; Wang, Z.; Chen, A.; Wright, P. K.; Evans, J. W. (2013). HighPerformance Dispenser Printed MA p-Type Bi ${ }_{0.5} \mathrm{Sb}_{1.5} \mathrm{Te}_{3}$ Flexible Thermoelectric Generators for Powering Wireless Sensor Networks. ACS Appl. Mater. Interfaces. 5, 11872-11876.

11. Lu, Z.; Layani, M.; Zhao, X.; Tan, L. P.; Sun, T.; Fan, S.; Yan, Q.; Magdassi, S.; Hng, H. H. (2014). Fabrication of flexible thermoelectric thin film devices by inkjet printing. Small. 10 (17), 3551-3554.

12. Juntunen, T.; Jussila, H.; Ruoho, M.; Liu, S.; Hu, G.; Albrow-Owen, T.; Ng, L. W. T.; Howe, R. C. T.; Hasan, T.; Sun, Z.; Tittonen, I. (2018). Inkjet Printed Large-Area Flexible Few-Layer Graphene Thermoelectrics. Adv. Funct. Mater. $28,1800480$.

13. Han, C.; Tan, G.; Varghese, T.; Kanatzidis, M. G.; Zhang, Y. (2018). HighPerformance PbTe Thermoelectric Films by Scalable and Low-Cost Printing. ACS Energy Lett. 3, 818-822.

14. Varghese, T.; Hollar, C.; Richardson, J.; Kempf, N.; Han, C.; Gamarachchi, P.; Estrada, D.; Mehta, R. J.; Zhang, Y. (2016). High-performance and flexible thermoelectric films by screen printing solution-processed nanoplate crystals. Sci. Rep. 6, 33135 .

15. Kim, S. J.; We, J. H.; Cho, B. J. (2014). A wearable thermoelectric generator fabricated on a glass fabric. Energy Environ Sci. 7 (6), 1959.

16. Kim, S. J.; Lee, H. E.; Choi, H.; Kim, Y.; We, J. H.; Shin, J. S.; Lee, K. J.; Cho, B. J. (2016). High-Performance Flexible Thermoelectric Power Generator Using Laser Multiscanning Lift-Off Process. ACS Nano. 10, 10851-10857.

17. Choi, H. W.; Zhou, T.; Singh, M.; Jabbour, G. (2015). Recent developments and directions in printed nanomaterials. Nanoscale. 7, 3338-3355. 
18. Sun, B. Y.; Rogers, J. A. (2007). Inorganic Semiconductors for Flexible Electronics. Adv Mater. 19, 1897-1916.

19. Oh, J. Y.; Lee, J. H.; Han, S. W.; Chae, S. S.; Bae, E. J.; Kang, Y. H.; Choi, W. J.; Cho, S. Y.; Lee, J.-O.; Baik, H. K.; Lee, T. Il. (2016). Chemically exfoliated transition metal dichalcogenide nanosheets-based wearable thermoelectric generators. Energy Environ Sci. 9, 1696-1705.

20. Dun, C.; Hewitt, C. A.; Li, Q.; Xu, J.; Schall, D. C.; Lee, H.; Jiang, Q.; Carroll, D. L. (2017). 2D Chalcogenide Nanoplate Assemblies for Thermoelectric Applications. Adv. Mater. 29, 1700070.

21. Wan, C.; Gu, X.; Dang, F.; Itoh, T.; Wang, Y.; Sasaki, H.; Kondo, M.; Koga, K.; Yabuki, K.; Snyder, G. J.; Yang, R.; Koumoto, K. (2015). Flexible n-type thermoelectric materials by organic intercalation of layered transition metal dichalcogenide $\mathrm{TiS}_{2}$. Nat. Mater. 14, 622-627.

22. Park, S. H.; Jo, S.; Kwon, B.; Kim, F.; Ban, H. W.; Lee, J. E.; Gu, D. H.; Lee, S. H.; Hwang, Y.; Kim, J. S.; Hyun, D. Bin; Lee, S.; Choi, K. J.; Jo, W.; Son, J. S. (2016). High-performance shape-engineerable thermoelectric painting. Nat. Commun. 7, 1-10.

23. Wan, C.; Tian, R.; Kondou, M.; Yang, R.; Zong, P.; Koumoto, K. (2017). Ultrahigh thermoelectric power factor in flexible hybrid inorganic-organic superlattice. Nat. Commun.. 8, 1024.

24. Dun, C.; Hewitt, C. A.; Li, Q.; Guo, Y.; Jiang, Q.; Xu, J.; Marcus, G.; Schall, D. C.; Carroll, D. L. (2017). Self-Assembled Heterostructures : Selective Growth of Metallic Nanoparticles on V2-VI3 Nanoplates. Adv Mater. 29, 1702968.

25. Du, Y.; Cai, K.; Chen, S.; Wang, H.; Shen, S. Z.; Donelson, R.; Lin, T. (2015). Thermoelectric Fabrics: Toward Power Generating Clothing. Sci. Rep. 5, 6411.

26. Du, Y.; Cai, K. F.; Chen, S.; Cizek, P.; Lin, T. (2014). Facile preparation and thermoelectric properties of $\mathrm{Bi}_{2} \mathrm{Te}_{3}$ based alloy nanosheet/PEDOT:PSS composite films. ACS Appl. Mater. Interfaces. 6 (8), 5735-5743. 
27. Wang, Y.; Cai, K.; Yao, X. (2011). Facile Fabrication and Thermoelectric Properties of PbTe-Modified Poly(3,4-ethylenedioxythiophene) Nanotubes. ACS Appl. Mater. Interfaces. 3 (4), 1163-1166.

28. Song, H.; Cai, K. (2017). Preparation and properties of PEDOT:PSS/Te nanorod composite films for flexible thermoelectric power generator. Energy. 125, 519525.

29. Du, Y.; Xu, J.; Paul, B.; Eklund, P. (2018). Flexible thermoelectric materials and devices. Appl. Mater. Today. 12, 366-388.

30. Han, C.; Sun, Q.; Li, Z.; Dou, S. X. (2016). Thermoelectric Enhancement of Different Kinds of Metal Chalcogenides. Adv. Energy Mater. 6, 1600498.

31. Fan, F. R.; Tang, W.; Wang, Z. L. (2016). Flexible Nanogenerators for Energy Harvesting and Self-Powered Electronics. Adv. Mater. 28, 4283-4305.

32. Bahk, J.-H.; Fang, H.; Yazawa, K.; Shakouri, A. (2015). Flexible thermoelectric materials and device optimization for wearable energy harvesting. J Mater Chem C. 3, 10362-10374.

33. Perelaer, J.; Smith, P. J.; Mager, D.; Soltman, D.; Volkman, S. K.; Subramanian, V.; Korvink, G.; Schubert, U. S. (2010). Printed electronics : the challenges involved in printing devices, interconnects, and contacts based on inorganic materials. J Mater Chem A. 20, 8446-8453.

34. Zhang, H.; Son, J. S.; Dolzhnikov, D. S.; Filatov, A. S.; Hazarika, A.; Wang, Y.; Hudson, M. H.; Sun, C.; Chattopadhyay, S.; Talapin, D. V. (2017). Soluble Lead and Bismuth Chalcogenidometallates: Versatile Solders for Thermoelectric Materials. Chem. Mater. 29, 6396-6404.

35. Son, J. S.; Zhang, H.; Jang, J.; Poudel, B.; Waring, A.; Nally, L.; Talapin, D. V. (2014). All-Inorganic Nanocrystals as a Glue for BiSbTe Grains: Design of Interfaces in Mesostructured Thermoelectric Materials. Angew. Chemie. 53, 7466 -7470 .

36. Poudel, B.; Hao, Q.; Ma, Y.; Lan, Y.; Minnich, A.; Yu, B.; Yan, X.; Wang, D.; Muto, A.; Vashaee, D.; Chen, X.; Liu, J.; Dresselhaus, M. S.; Chen, G.; Ren, Z. 
(2008). High-thermoelectric performance of nanostructured bismuth antimony telluride bulk alloys. Science. 320, 634-638.

37. Liu, Y.; Zhang, Y.; Ortega, S.; Ibáñez, M.; Lim, K. H.; Grau-Carbonell, A.; Martí-Sánchez, S.; Ng, K. M.; Arbiol, J.; Kovalenko, M. V.; Cadavid, D.; Cabot, A. (2018). Crystallographically Textured Nanomaterials Produced from the Liquid Phase Sintering of $\mathrm{Bi}_{\mathrm{x}} \mathrm{Sb}_{2-\mathrm{x}} \mathrm{Te}_{3}$ Nanocrystal Building Blocks. Nano Lett. $18,2557-2563$.

38. Kim, Y. M.; Lydia, R.; Kim, J.; Lin, C.; Ahn, K.; Rhyee, J. (2017). Enhancement of thermoelectric properties in liquid-phase sintered Te-excess bismuth antimony tellurides prepared by hot-press sintering. Acta Mater. 135, 297-303.

39. Wang, L.; Zhang, Z.; Liu, Y.; Wang, B.; Wang, S. (2018). Exceptional thermoelectric properties of flexible organic-inorganic hybrids with monodispersed and periodic nanophase. Nat. Commun. 9, 3817.

40. Macleod, B. A.; Stanton, N. J.; Gould, I. E.; Wesenberg, D.; Ihly, R.; Owczarczyk, Z. R.; Hurst, K. E.; Fewox, C. S.; Folmar, C. N.; Hughes, H.; Zink, B. L.; Blackburn, L.; Ferguson, A. J. (2017). Large n- and p-type thermoelectric power factors from doped semiconducting single-walled carbon nanotube thin films. Energy Environ Sci. 10, 2168-2179.

41. Toshima, N.; Oshima, K.; Anno, H.; Nishinaka, T.; Ichikawa, S.; Iwata, A.; Shiraishi, Y. (2015). Novel Hybrid Organic Thermoelectric Materials:ThreeComponent Hybrid Films Consisting of a Nanoparticle Polymer Complex, Carbon Nanotubes, and Vinyl Polymer. Adv. Mater. 27, 2246-2251.

42. Kim, G.-H.; Shao, L.; Zhang, K.; Pipe, K. P. (2013). Engineered doping of organic semiconductors for enhanced thermoelectric efficiency. Nat. Mater. 12 (8), 719-723.

43. Yang, C.; Souchay, D.; Knei?, M.; Bogner, M.; Wei, H. M.; Lorenz, M.; Oeckler, O.; Benstetter, G.; Fu, Y. Q.; Grundmann, M. (2017). Transparent flexible thermoelectric material based on non-toxic earth-abundant p-type copper iodide thin film. Nat. Commun. 8, 16076. 
44. Paul, B.; Bjo, E. M.; Kumar, A.; Lu, J.; Eklund, P. (2018). Nanoporous $\mathrm{Ca}_{3} \mathrm{Co}_{4} \mathrm{O}_{9}$ Thin Films for Transferable Thermoelectrics. ACS Appl. Mater. Interfaces. 1, 2261-2268.

45. Lea, P.; (2018). Internet of Things for Architects (Packt Publishing).

46. Yee, S. K.; LeBlanc, S.; Goodson, K. E.; Dames, C. (2013). \$ per W metrics for thermoelectric power generation: beyond ZT. Energy Environ Sci. 6 (9), 25612571.

47. Ruoho M, Valset K, Finstad T and Tittonen I. (2015). Measurement of thin film thermal conductivity using the laser flash method. Nanotechnology 26 


\section{CHAPTER FOUR - AEROSOL JET PRINTING AND PHOTONIC SINTERING OF COLLOIDAL THERMOELECTRIC NANOCRYSTALS}

\subsection{Introduction}

Chapters 2 and 3 described the formulation of thermoelectric inks for screen printing processes. A screen printing process is a high through put process but it is limited to 2D substrates and also the dimensional resolution for legs are limited. Here we report a novel aerosol jet printing (AJP) capable of producing two dimensional flexible and 3D conformal printing of thermoelectric materials with a higher resolution. These capabilities help to print directly on the surface of objects instead of printing on flexible substrates and wrapping it around objects. Aerosol Jet Printing is capable of printing dimensions as small as $10 \mu \mathrm{m}$, and capable of producing fine features not only help to improve packing density but also improve the power density of the devices.

Thermoelectric properties of the material highly depends upon the sintering conditions of the printed materials but because of the low melting temperature of plastic substrates the sintering temperature and option for using different substrates are limited. Here we report a new study on photonic sintering or intense pulse light sintering of thermoelectric materials using a Xenon pulse light source. This opens a new door to printing thermoelectric material on wide variety of substrates and allow them to be sintered without deforming or destroying the substrates with shorter amounts of time. Combining aerosol jet printing and photonic sintering helps to create roll to roll printing opportunities for thermoelectric devices considerably reducing the cost of manufacturing 
and also reducing the time for fabrication. AJP not only helps to print fine dimensions but also helps to control the thickness of the printed lines, which is a crucial factor in optimizing photonic sintering parameters.

\subsection{Background}

Thermoelectric materials are highly efficient in collecting waste heat and transforming them in to electrical power output using a material property known as the Seebeck coefficient. The waste heat can be obtained by industrial activities, machine operations, combustion of fossil fuels and even human body heat. There is tremendous demand for thermoelectric materials for this exploding time of self-powered sensors and wearable devices for Internet of Things (IoT) and smart wearable applications ${ }^{1}$. This also brought a lot of new challenges in providing self- powered solutions that can be easily integrated and that are comfortable, light and flexible. Even an average human body can dissipate $\sim 100 \mathrm{~W}$ of heat to their surroundings under normal circumstances ${ }^{2}$, a wearable thermoelectric generator can be used as an energy source for low power sensor applications if they are implanted in clothing or worn as patches which cover typically hotter regions of the body such as the forehead and chest ${ }^{3}$. Recently other researchers have demonstrated utilizing screen printing, Ink jet printing and spin coating ${ }^{4}$ to develop flexible thermoelectric films with high performance ${ }^{5-7}$. Additive printing of the flexible thermoelectric generators offers a good solution to improving low cost and efficient energy harvesting methods. But manufacturing high power density thermoelectric devices that can operate at low temperature are extremely difficult and also the manufacturing processes are limited to $2 \mathrm{D}$ printing of thermoelectric materials, whereas to utilize the complete potential of the material a $3 \mathrm{D}$ conformal printing is highly useful ${ }^{8}$. 
Kim F. et al reported extrusion based three dimensional printing methods to produce thermoelectric material with geometries is suitable for heat sources and successful in obtaining bulk like thermoelectric properties. It offers a potential solution to the dicing and wastage associated with bulk thermoelectric materials, but has drawbacks associated with the assembly of the materials to the temperature sources. 3 -axis direct write printing and Aerosol Jet printing are two instruments capable of conformal printing onto any 3D objects providing opportunities to print multi layers of materials on 3D objects directly and sinter them to obtain conformably printed thermoelectric generators. Among these aerosol jet printing is highly efficient in creating thin film with minimal material usage and very high precision.

The ZT factor of TE generators can be significantly enhanced of TE materials using nanoparticles due to the reduction in lattice thermal conductivity. High performance TE films have been demonstrated by printing nanoparticles followed by thermal sintering at around $400^{\circ} \mathrm{C}$ in an inert environment ${ }^{7}$. However, conventional sintering methods such as thermal sintering in the furnace suffer from two major limitations. First the process of thermal sintering can take several hours per batch making it unsuitable for rapid fabrication methods such as roll- to roll manufacturing of TE generators. Second, conversational thermal sintering exposes both the printed film and substrate to high temperature which limits the type of substrate that can be used to form the films ${ }^{9}$. To overcome these challenges we demonstrate in this work the use of a photonic sintering method to create TE films from Bismuth Telluride based nanoparticles where sintering/ densification is achieved over large areas ( several square inches), in extremely short periods of time ( milliseconds per pulse) and using a rigid glass as well as 
flexible polymer substrate. Due to the high speed of sintering, this method is highly compatible with rapid roll-to roll manufacturing of low cost printed TE generators for applications such as energy harvesting. $\mathrm{Bi}_{2} \mathrm{Se}_{0.3} \mathrm{Te}_{2.7}$ thermoelectric material reported to have the highest $\mathrm{ZT}$ at room temperature compared to any other materials ${ }^{9}$. This work focuses on aerosol jet printing and photonic sintering studies of $\mathrm{Bi}_{2} \mathrm{Se}_{0.3} \mathrm{Te}_{2.7}$ based $\mathrm{TE}$ nanoparticles. To the best of our knowledge no one has reported to date on aerosol jet printed and photonic sintered TE device fabrication.

\subsection{Materials and Methods}

\subsubsection{Thermoelectric Ink for Aerosol Jet Printing}

$\mathrm{Bi}_{2} \mathrm{Te}_{2.8} \mathrm{Se}_{0.3}$ nanoparticles synthesized using a microwave stimulated wetchemical method were used for $\mathrm{N}$ type ink. $40 \mathrm{wt} \%$ of the nanoparticles were added to $60 \mathrm{wt} \%$ of solvent mix consisting of ethylene glycol: glycerol and ethanol (35:5:60 wt \%). The powder is slowly added into the solvent mixture under magnetic stirring at room temperature. The solution is kept under magnetic stirring for 2 hours, followed by bath sonication for 3 hours at room temperature. TGA capping on the nanomaterials help the dispersion of the nanoparticles in the solvent mixture. In addition, the ink atomization process in the pneumatic atomizer sets up itself use a magnetic stirring and a vigorous stirring is applied, which helps to improve the homogenous jetting of the inks.

\subsubsection{Fabrication of Aerosol Jet Printed Thermoelectric Films}

$40 \mathrm{ml}$ of the thermoelectric ink is loaded in to the pneumatic atomizer of the Optomec aerosol jet printer 300, as Figure $\mathbf{1 . 6}$ shows, $\mathrm{N}_{2}$ gas will carry the aerosol from the chamber to the nozzle. Developing inks for pneumatic atomizers helps to have more nanoparticle loading as well as high viscosity inks for the aerosol jet printing process, 
reducing porosity and density for the printed films. Moreover, pneumatic atomizers can magnetically stir the ink and increase the temperature of the ink leading to a uniform and uninterrupted flow of jet through the nozzle. Once the ink is aerosolized it passes through a trap, where larger droplets are collected before going to the nozzle and preventing clogging of the inks at the nozzle. The aerosol jet droplets were carried by $\mathrm{N}_{2}$ gas and deposited on to an oxygen plasma treated (90W 10 minutes) HN Kapton substrates (51 $\mu \mathrm{m}$ thick) kept under a table temperature of $75^{\circ} \mathrm{C}$. The sheet gas of $\mathrm{N}_{2}$ helps to avoid clogging and focusing of the printed lines. The processing speed and sheath gas flow rate arecontrolled to get the desired feature dimensions.

The printing parameters for different inks are summarized in Table 4.1. The parameters were optimized to have smooth and fast printing of the thermoelectric inks. A 300-micron nozzle is used to print the inks, the nozzle and the substrates are kept at $3 \mathrm{~mm}$ distance. Once the printed lines are optimized a CAD model of the required structures is developed with an overlap of 30 to $40 \%$ and uses a serpentine filling parameters. The filling parameters and serpentine filling in the structures helps to avoid the ridges and valleys associated with the printed structures as shown in Figure 4.1

\subsubsection{Photonic Sintering of The Printed Films}

A xenon flash lamp was used for the photonic sintering (Xenon S2100) of the TE films. First the printed thermoelectric films are dried at $200^{\circ} \mathrm{C}$ for 5 minutes using a hot plate. Once the films are cooled down, the films undergo a cold compaction using a hydraulic press up to $10 \mathrm{Klbf}$ using suitable cover plates. The pressed films were then mounted on to flash lamp chamber and secured using scotch tape. The distance between the spiral lamp and the sample surface are kept at 1 inch, to obtain maximum power 


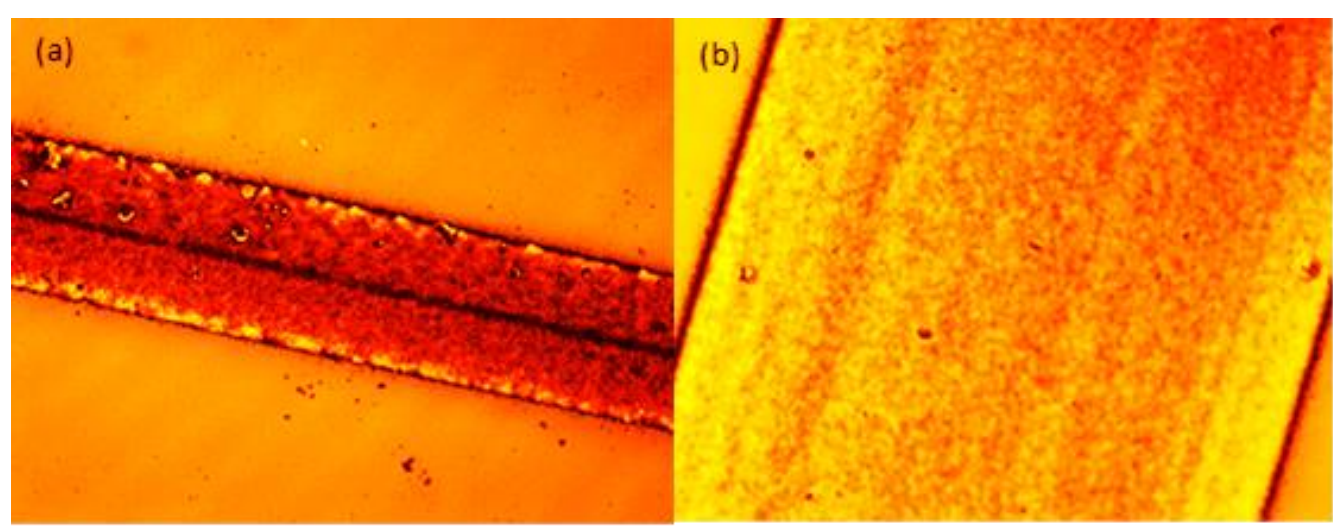

Figure 4. 1 (a) Perimeter filled AJP prints (b) Serpentine filled AJP prints with 30$40 \%$ overlapping

Table 4. 1 Summary of the aerosol jet printing parameters

\begin{tabular}{|c|c|}
\hline Ink & Printing parameters \\
\hline $\mathrm{N}$ type- $\mathrm{Bi}_{2} \mathrm{Te}_{2.7} \mathrm{Se}_{0.3}$ & Pneumatic atomizer : 500 \\
& Pneumatic exhaust: 470 \\
& Sheath gas flow: 80 \\
& Processing speed: 2.5 \\
\hline
\end{tabular}

output of the flash light and the pulsing was carried out in a $\mathrm{N}_{2}$ gas flow. The wave length of the xenon lamp ranged from 200 to $800 \mathrm{~nm}$, with a high intensity for wavelengths of 400-500 nm. Sample performance was optimized by tuning the pulse energy, duration, delay time and number of pulses. The morphology of the samples were studied using optical imaging and a scanning electron microscope. The sintered films also were characterized by measuring their electrical conductivity and Seebeck coefficient with the measurements done using four point probe measurement and a custom made Seebeck set up. 


\subsection{Results and Discussion}

\subsubsection{Ink Characteristics}

Optimized thermoelectric ink is characterized for its adhesion properties, contact angle measurements were carried out on Kapton substrates using Biolin Scinetific Attention Theta Optical Tensiometeter. HN Kapton substrates were cleaned using IPA, methanol, acetone mix and dried; the inks were deposited before and after oxygen plasma shown in Figure 4.2. A contact angle of $34.64^{\circ}$ is obtained for Kapton substrates with no surface treatment as shown in Figure 4.2 1(a), there is a non-uniformity in the observed contact angle. After the substrate is treated with oxygen plasma of $90 \mathrm{~W}$ for 10 minutes, a clear difference is observed in the surface properties of the ink on the Kapton. The ink shows more wettability and the contact angle got reduced to $20.36^{\circ}$ and a uniform wetting of the ink is also observed. The improvement in contact angle shows a direct relation to the improvement in adhesion of the inks during aerosol jet printing. Improvement in adhesion is a key factor in successful sintering of the samples under a high energy short pulse light source. The thermoelectric materials and the polymer substrates have a different coefficient of thermal expansion rate (CTE), this large mismatch in CTE could destroy the printed films. An improvement in thermoelectric material adhesion to the substrate helped in the photonic sintering of the films without destroying them.

The optimized inks are printed on to different substrates as shown in Figure 4.3 (a) \& (b) Kapton sheets and photo papers respectively showing improved adheshion on the surface after oxygen plasma treatment. Figure 4.3 (c) shows AJP printed single leg Ntype thermoelectric devices on Kapton substrates, with superior flexibility allowing the 
devices to be rolled as compared to a penny. Figure 4.3(d) shows a conformal printed N type legs onto silver contacts deposited on glass substrates showing good adheshion and alignment of the second layer observed on the printed films.
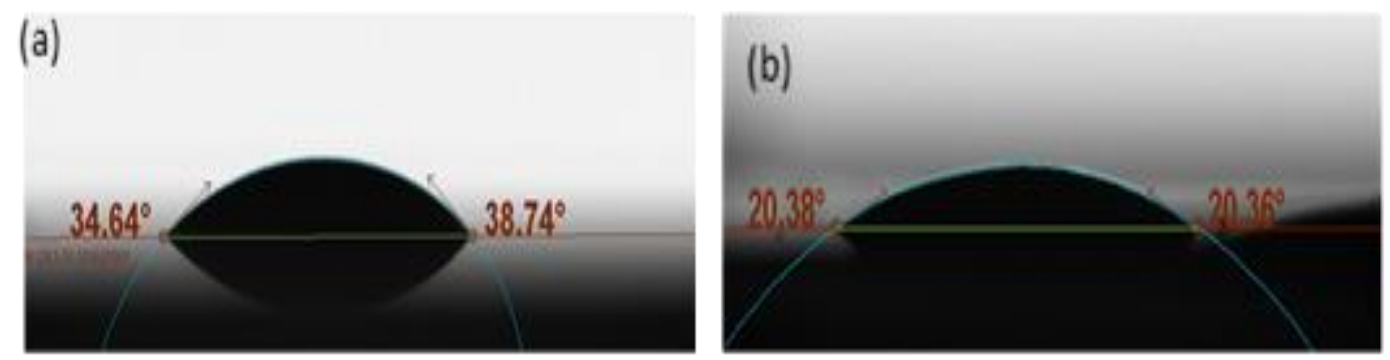

Figure 4. 2 Contact angle measurements of the AJP thermoelectric inks (a) cleaned Kapton substrate (b) Oxygen plasma treated Kapton substrate
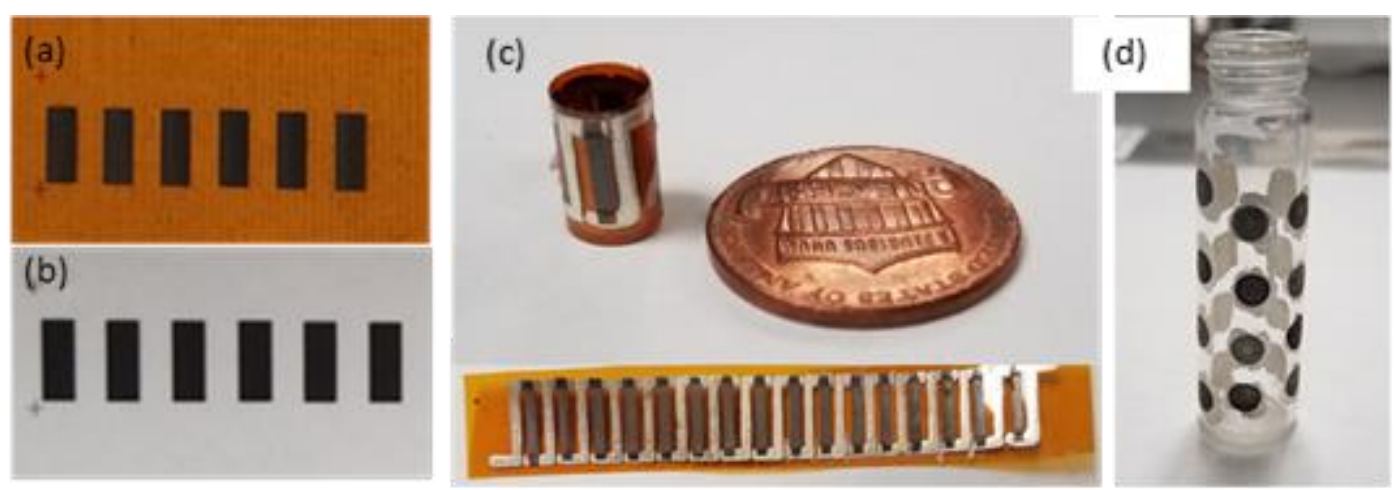

Figure 4.3 (a) \& (b) AJP printed samples on Kapton and Photopaper,(c) AJP printed photonic sintered N-type thermoelectric device,(d) 3D conformal printed thermoelectric devices on silver contacts.

Aerosol jetting is a spraying technique and forms random stacking of thermoelectric materials creating a large amount of porosity and potentially trapping solvents in the films creating cracks during the drying process. Electrical conductivity measurements showed high resistance for these films and non-uniform sintering of the films due to less contact surface of the films. To further improve the thermoelectric properties of the films, the films were subjected to a cold compaction help to reduce the porosity and also improve the contact area between the nanocrystals. Figure 4.4 (a) 
shows the 3D mapping of the AJP printed thermoelectric legs, a random stacking of material can be observed creating a rough surface for the films. Figure 4.4 (b) shows the surface mapping of the films after cold compaction using a hydraulic press. The sample is sandwiched between two high purity mica sheets (TED pella.inc) and 10Klbf is applied and the samples are carefully separated from the mica sheets. The 3D mapping after cold compaction shows a uniform surface finish and compaction throughout the surface of the films reducing porosity and improvement in TE properties for the films. The cold compaction not only improves surface finish but also helps to densify the films, improving nanoparticle contact areas and reducing porosity. The thickness of the films reduce $40-45 \%$ after cold compaction compared to the printed films.
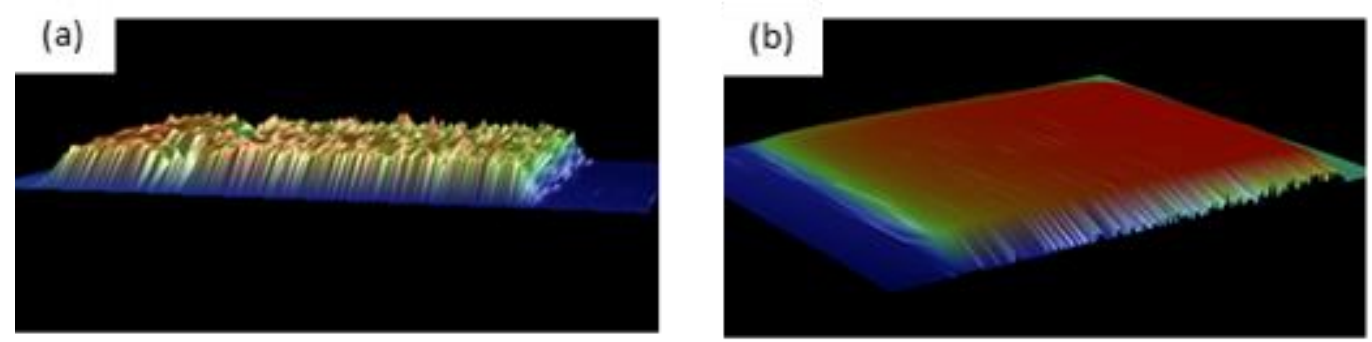

Figure 4. 4 3D mapping of the aerosol jet printed sample (a) dried sample with no pressure applied (b) sample after $10 \mathrm{KIbf}$ force applied using a hydraulic press.

Hydraulic pressed films are carefully mounted on to the photonic sintering chamber, an inert ambiance of $\mathrm{N}_{2}$ is applied in the chamber using special attachments. The chamber is purged with $\mathrm{N}_{2}$ gas for 5 minutes to reduce the oxygen presence in the chamber. Samples are then bombarded with high energy pulses varying the duration and number of pulses.

Figure 4.5 (a) shows the surface image of $\mathrm{Bi}_{2} \mathrm{Se}_{0.3} \mathrm{Te}_{2.7}$ film, showing large number of pores in the films. Figure 4.5 (b) \& (c) shows the cross sectional images of the sintered films with random arrangements of the nanocrystals. Big pores are present in 
the sample due to rapid solvent evaporation and high energy pulse. Further improvement in density is possible with more detailed studies in optimizing photonic sintering parameters. Thermoelectric properties were studied varying the energy of the pulses, duration and number of pulses applied on the samples. Figure 4.6 shows the thermoelectric properties of the printed films as a function of the number of pulses, electrical conductivity of the printed films increases as the number of pulses. A peak electrical conductivity of $445 \mathrm{~S} / \mathrm{cm}$ is observed at 12 number of pulses. Similar results were obtained for Seebeck coefficients reaching a peak Seebeck coefficient of $-80 \mu \mathrm{V} / \mathrm{K}$ is obtained at 15 pulses. Electrical conductivity of the films shows comparable results as that of other published results whereas the Seebeck coefficient is lower compared to other thin films.
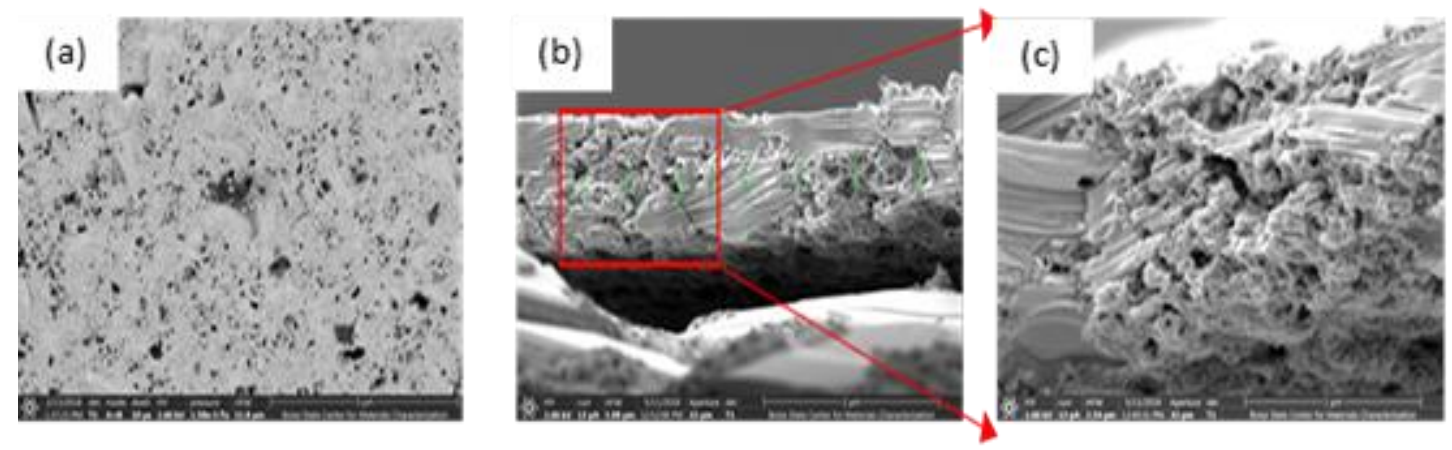

Figure 4. 5 SEM images (a) surface images of $\mathrm{Bi}_{2} \mathrm{Te}_{3}$ photonic sintered sample and (b-c) cross section of a printed film on polyimide substrate AJP printed and photonic sintered.

Decrease in the Seebeck coefficient of photonic sintered material due to a large number of porosity in the film and formation of oxidation layers on the material under sintering conditions further optimization in the procedure are required to improve the properties. A maximum power factor $270 \mu \mathrm{W} / \mathrm{mK}^{2}$ is obtained at 12 pulses for the films 
with an average thickness $1.38 \mu \mathrm{m}$. The result is comparable to other flexible thermoelectric films manufactured using ink jet printing and screen printing.
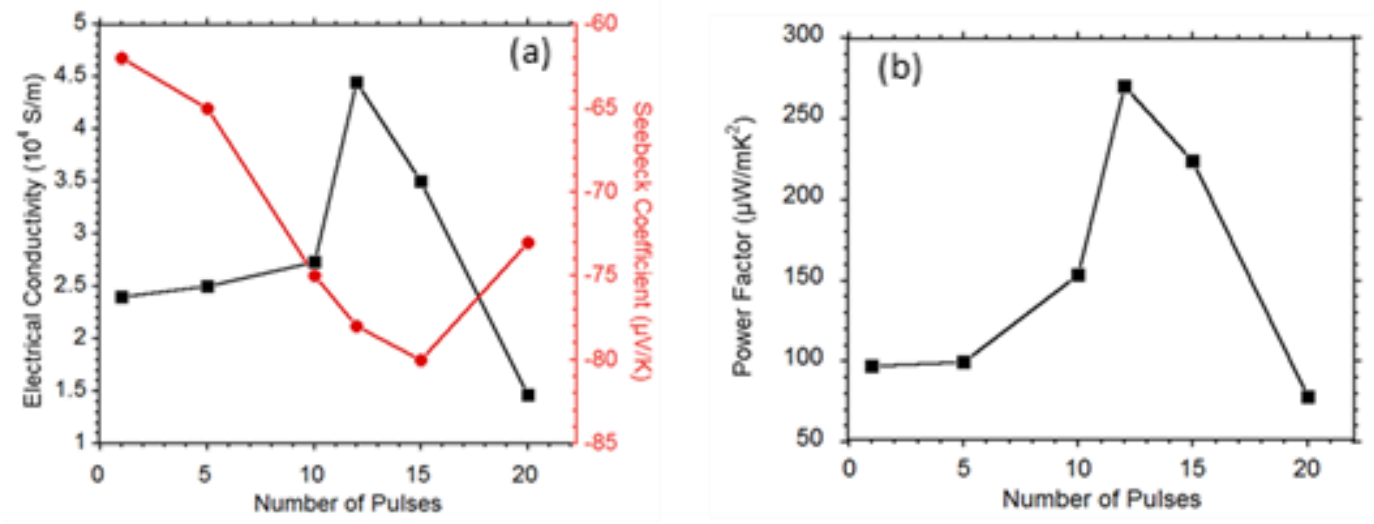

Figure 4. 6 (a) electrical conductivity and Seebeck coefficient as a function of number of pulses (b) power factor vs number of pulses.

\subsection{Conclusion}

This work shows the first demonstration of an Aerosol jet printed, flexible $\mathrm{Bi}_{2} \mathrm{Se}_{0.3} \mathrm{Te}_{2.7}$ films with photonic sintering. Highly flexible thermoelectric legs with an electrical conductivity of $445 \mathrm{~S} / \mathrm{cm}$ is obtained, comparable to $\mathrm{N}$ type flexible thermoelectric materials manufactured using other printing methods. This result shows promise of highly efficient, scalable and low cost methods to transform high-efficiency TE nanoparticles to economically viable thermoelectric devices for thermal energy harvesters for wearable applications or in applications of surface conformability of the energy harvesters.

\subsection{References}

1 Fraga-Lamas, T. M. F.-C. a. P. Towards The Internet of Smart Clothing: A Review on IoT Wearables and Garments for Creating Intelligent Connected ETextiles. Electronics, doi:doi.org/10.3390/electronics7120405 (2018).

2 Suarez, F., Nozariasbmarz, A., Vashaee, D. \& Öztürk, M. C. Designing thermoelectric generators for self-powered wearable electronics. Energy \& 
Environmental Science 9, 2099-2113, doi:10.1039/C6EE00456C (2016)Lin, Z. et al. A Solution Processable High-Performance Thermoelectric Copper Selenide Thin Film. Advanced Materials 29, 1606662, doi:10.1002/adma.201606662 (2017).

3. Bhatia, D., Bairagi, S., Goel, S. \& Jangra, M. Pacemakers charging using body energy. Journal of Pharmacy And Bioallied Sciences 2, 51-54, doi:10.4103/09757406.62713 (2010).

4 Bubnova, O. et al. Optimization of the thermoelectric figure of merit in the conducting polymer poly(3,4-ethylenedioxythiophene). Nature Materials 10, 429,doi:10.1038/nmat3012https://www.nature.com/articles/nmat3012\#supplemen tary-information (2011).

5 Madan, D. et al. Enhanced Performance of Dispenser Printed MA n-type Bi2Te3 Composite Thermoelectric Generators. Acs Appl Mater Inter 4, 6117-6124, doi:10.1021/am301759a (2012).

6 Varghese, T. et al. High-performance and flexible thermoelectric films by screen printing solution-processed nanoplate crystals. Sci Rep-Uk 6, doi:ARTN $3313510.1038 /$ srep33135 (2016).

7 Kim, F. et al. 3D printing of shape-conformable thermoelectric materials using all-inorganic Bi2Te3-based inks. Nature Energy 3, 301309,doi:10.1038/s41560017-0071-2 (2018).

8 Danaei, R. et al. Ultrafast Fabrication of Thermoelectric Films by Pulsed Light Sintering of Colloidal Nanoparticles on Flexible and Rigid Substrates. Advanced Engineering Materials 21, 1800800, doi:10.1002/adem.201800800 (2019).

9 Kim, S. I. et al. Dense dislocation arrays embedded in grain boundaries for highperformance bulk thermoelectrics. Science 348, 109-114, doi:10.1126/science.aaa4166 (2015). 


\section{CHAPTER 5 SUMMARY AND FUTURE WORK}

\section{$5.1 \quad$ Summary}

Low temperature thermoelectric energy harvesters have received significant research attention due to recent advancement in IoT and wearable devices. TEG are capable of utilizing low grade heat energy and convert electrical energy in applications such as hot pipes, car exhausts, heat engines and nuclear waste materials. This work presents the development of large scale flexible thermoelectric devices resembling the same performance as that of bulk legs and optimized ink compositions and sintering conditions to improve the ZT of $\mathrm{n}$ and $\mathrm{p}$ - type thermoelectric inks for screen printing. A prototype TEG was printed to obtain a high power density of $18.8 \mathrm{~mW} / \mathrm{cm}^{2}$ similar to that of bulk counter parts and a novel aerosol jet printing approaches to print micro thermoelectric generators on 2D flexible substrate as well as on 3D surfaces. A roll to roll manufacturing is initiated utilizing intense pulse light sintering for thermoelectric materials on low temperature substrates.

Findings of this work are summarized below

1. Direct conversion of wet chemical synthesized colloidal nanocrystals into a flexible thermoelectric generator is obtained using screen a printing process

2. Screen printed $\mathrm{N}$ type $\mathrm{Bi}_{2} \mathrm{Se}_{0.2} \mathrm{Te}_{2.8}$ flexible thermoelectric generators have developed with a peak ZT of 0.43

3. Bulk like performing $\mathrm{P}$ type $\mathrm{Bi}_{1.6} \mathrm{Sb}_{0.4} \mathrm{Te}_{3}$ flexible thermoelectric devices with peak ZT of 1 are achieved using screen printing and liquid phase sintering 
4. Highly scalable and interchangeable ink composition is developed and is demonstrated in the fabrication of BiTe and $\mathrm{PbTe}$ thermoelectric generators

5. Novel photonic sintering studies were performed in $\mathrm{N}$ type $\mathrm{Bi}_{2} \mathrm{Se}_{0.2} \mathrm{Te}_{2.8}$ flexible thermoelectric generators for roll- roll to fabrication of thermoelectric generators

6. New ink composition and aerosol jet printing processes have been developed for 2D flexible micro thermoelectric generators and 3D conformal printing of thermoelectric generators.

Based on these findings it is clear that the following three factors have the most influence on printed TE film properties:

\subsection{Future Work}

Wet chemical synthesis of thermoelectric nanocrystals ink consisting of nanocrystals with varying thickness, controlling the process parameters and implementation of density gradient centrifugation (DGU) techniques could help to separate single crystals of nanomaterials from the ink for printing. Single crystal nanomaterial will lead to drastic improvements in its thermoelectric figure of merit, improving the quantum confinement of charge carriers and reduction of thermal conductivity ${ }^{1}$. Thermoelectric properties, especially thermal conductivity of the printed thermoelectric materials can be controlled considerably by engineering the nanocrystal interfaces and recently inorganic capping agents are gaining a lot of attention for this method $^{2-3}$. AJP printing and photonic sintering of inorganic capped nanocrystals could prove to improve the TE properties by managing the interfaces as well as reducing the porosity in the films. 
Additive manufacturing methods proved to be capable of reducing the cost of manufacturing TEG as close to the cost of materials, the inorganic materials used in the fabrication are expensive and toxic. Development of new cheap and high performing thermoelectric materials are necessary to further reduce the cost of thermoelectric generators and safety of the devices, especially in applications related to health care and medical implantable devices. Recent researches on polymer based thermoelectric and nontoxic inorganic material ${ }^{4}$ are a good examples of less toxic and high performing new thermoelectric materials. Especially organic thermoelectric generators with their low cost, flexibility and non-toxicity make them appealing in wearable and stretchable electronic applications ${ }^{5}$. Even bulk like performance was achieved for the printed flexible thermoelectric generators, the power produced using these materials are low because of high contact resistance associated with the device fabrications. By optimizing the interface materials and metal contact layer this parasitic resistance can be reduced and improve the performance of flexible thermoelectric generators In-plane and cross plane devices.

\subsection{References}

1. Teweldebrhan, D.; Goyal, V.; Balandin, A. A., Exfoliation and Characterization of Bismuth Telluride Atomic Quintuples and Quasi-Two-Dimensional Crystals. Nano Letters 2010, 10 (4), 1209-1218.

2. Park, S. H.; Jo, S.; Kwon, B.; Kim, F.; Ban, H. W.; Lee, J. E.; Gu, D. H.; Lee, S. H.; Hwang, Y.; Kim, J.-S.; Hyun, D.-B.; Lee, S.; Choi, K. J.; Jo, W.; Son, J. S., High-performance shape-engineerable thermoelectric painting. Nature Communications 2016, 7, 13403. 
3. Kim, F.; Kwon, B.; Eom, Y.; Lee, J. E.; Park, S.; Jo, S.; Park, S. H.; Kim, B.-S.; Im, H. J.; Lee, M. H.; Min, T. S.; Kim, K. T.; Chae, H. G.; King, W. P.; Son, J. S., 3D printing of shape-conformable thermoelectric materials using all-inorganic Bi2Te3-based inks. Nature Energy 2018, 3 (4), 301-309.

4. Wan, C.; Gu, X.; Dang, F.; Itoh, T.; Wang, Y.; Sasaki, H.; Kondo, M.; Koga, K.; Yabuki, K.; Snyder, G. J.; Yang, R.; Koumoto, K., Flexible n-type thermoelectric materials by organic intercalation of layered transition metal dichalcogenide TiS2. Nature Materials 2015, 14, 622.

5. Russ, B.; Glaudell, A.; Urban, J. J.; Chabinyc, M. L.; Segalman, R. A., Organic thermoelectric materials for energy harvesting and temperature control. Nature Reviews Materials 2016, 1, 16050. 